\title{
Classification of a Diverse Set of Tetrahymena Pyriformis Toxicity Chemical Compounds from Molecular
}

\section{Descriptors by Statistical Learning Methods}

\author{
Y. Xue ${ }^{1,3}$, H. Li ${ }^{1}$, C.Y. Ung ${ }^{1,2,}$ C.W. Yap ${ }^{1}$ and Y.Z. Chen ${ }^{*}, 1$
}

${ }^{1}$ Bioinformatics and Drug Design Group, Department of Pharmacy and Department of Computational Science, National University of Singapore, Blk S16, Level 8, 3 Science Drive 2, Singapore 117543

${ }^{2}$ Department of Biochemistry, The Yong Loo Lin School of Medicine, National University of Singapore, Blk MD7, \#02-03, 8 Medical Drive, Singapore 117597.

\footnotetext{
${ }^{3}$ College of Chemistry, Sichuan University, Chengdu, 610064, P. R. China
} 
Table S1. All the compounds can be found in the listed references. In this study, agents were divided into TPT and non-TPT groups according to whether the $\log (1 / \mathrm{IGC} 50)$ value is $>-0.5$ or $\leq-0.5$ respectively. $\mathrm{n}$ : non-toxic chemical in this work; $\mathrm{p}$ : toxic chemical in this work.

\begin{tabular}{|c|c|c|c|c|c|c|}
\hline No. & Class & CAS number & Name & Smile Name & $\begin{array}{c}\log \\
\left(\text { IGC }_{50}\right)^{-1}\end{array}$ & references \\
\hline 1 & $\mathrm{n}$ & $100-51-6$ & benzyl alcohol & $\mathrm{C}(\mathrm{O}) \mathrm{c} 1 \mathrm{ccccc} 1$ & -0.83 & 1,2 \\
\hline 2 & $\mathrm{p}$ & $100-46-9$ & benzylamine & $\mathrm{C}(\mathrm{N}) \mathrm{c} 1 \mathrm{ccccc} 1$ & -0.24 & 1,2 \\
\hline 3 & $\mathrm{p}$ & $104-86-9$ & 4-chlorobenzamide & $\mathrm{C}(\mathrm{N}) \mathrm{c} 1 \mathrm{ccc}(\mathrm{cc} 1) \mathrm{Cl}$ & 0.16 & 1,2 \\
\hline 4 & $\mathrm{p}$ & $106-47-8$ & 4-chloroaniline & Clc1ccc $(\operatorname{cc} 1) \mathrm{N}$ & 0.05 & $1,2,14$ \\
\hline 5 & $\mathrm{n}$ & $98-85-1$ & sec-phenethyl alcohol & $\mathrm{C}[\mathrm{C} @ @ \mathrm{H}](\mathrm{O}) \mathrm{c} 1 \mathrm{cccc} 1$ & -0.66 & 1 \\
\hline 6 & $\mathrm{p}$ & $95-51-2$ & 2-chloroaniline & Clc1c(N)ccce1 & -0.17 & $1,2,14$ \\
\hline 7 & $\mathrm{p}$ & $122-97-4$ & 3-phenyl-1-propanol & $\mathrm{C}(\mathrm{CCO}) \mathrm{c} 1 \mathrm{ccccc} 1$ & -0.21 & 1,2 \\
\hline 8 & $\mathrm{p}$ & $108-42-9$ & 3-chloroaniline & $\mathrm{Clc1} \operatorname{cc}(\mathrm{N}) \operatorname{ccc} 1$ & 0.22 & 1,2 \\
\hline 9 & $\mathrm{p}$ & $120055-09-6$ & 1-phenyl-2-butanol & $\mathrm{C}([\mathrm{C} @ \mathrm{H}](\mathrm{CC}) \mathrm{O}) \mathrm{c} 1 \mathrm{ccccc} 1$ & -0.16 & 1 \\
\hline 10 & $\mathrm{p}$ & $529-19-1$ & 2-tolunitrile & $\mathrm{C}(\# \mathrm{~N}) \mathrm{c} 1 \mathrm{c}(\mathrm{C}) \mathrm{cccc} 1$ & -0.24 & $1,2,3$ \\
\hline 11 & $\mathrm{p}$ & $100-66-3$ & methoxybenzene & $\mathrm{O}(\mathrm{C}) \mathrm{c} 1 \mathrm{ccccc} 1$ & -0.10 & 1,2 \\
\hline 12 & $\mathrm{p}$ & $100-44-7$ & benzyl chloride & $\mathrm{C}(\mathrm{Cl}) \mathrm{c} 1 \mathrm{ccccc} 1$ & 0.10 & 1,2 \\
\hline 13 & $\mathrm{p}$ & $2722-36-3$ & 3-phenyl-1-butanol & $\mathrm{C}([\mathrm{C} @ \mathrm{H}](\mathrm{C}) \mathrm{c} 1 \mathrm{ccccc} 1) \mathrm{CO}$ & 0.01 & 1 \\
\hline 14 & $\mathrm{p}$ & $615-65-6$ & 2-chloro-4-methylaniline & $\operatorname{Clcl}(\mathrm{N}) \operatorname{ccc}(\mathrm{c} 1) \mathrm{C}$ & 0.18 & 1,2 \\
\hline 15 & $\mathrm{p}$ & $71-43-2$ & benzene & $\mathrm{c} 1 \mathrm{ccccc} 1$ & -0.12 & 1 \\
\hline 16 & $\mathrm{p}$ & $623-12-1$ & 4-chloroanisole & $\mathrm{O}(\mathrm{C}) \mathrm{c} 1 \mathrm{ccc}(\mathrm{cc} 1) \mathrm{Cl}$ & 0.60 & 1,2 \\
\hline 17 & $\mathrm{p}$ & $768-59-2$ & 4-ethylbenzyl alcohol & $\mathrm{C}(\mathrm{C}) \mathrm{c} 1 \mathrm{ccc}(\mathrm{cc} 1) \mathrm{CO}$ & 0.07 & 1,2 \\
\hline 18 & $\mathrm{p}$ & $500-66-3$ & olivetol & $\mathrm{C}(\mathrm{CCCC}) \mathrm{c} 1 \mathrm{cc}(\mathrm{O}) \mathrm{cc}(\mathrm{c} 1) \mathrm{O}$ & 1.31 & $1,2,4,5,7,8,11,13$ \\
\hline 19 & $\mathrm{p}$ & $1565-75-9$ & $( \pm)$-2-phenyl-2-butanol & $\mathrm{C}([\mathrm{C} @](\mathrm{C})(\mathrm{O}) \mathrm{c} 1 \mathrm{ccccc} 1) \mathrm{C}$ & 0.06 & 1 \\
\hline 20 & $\mathrm{p}$ & $136-77-6$ & 4-hexylresorcinol & $\mathrm{C}(\mathrm{CCCCC}) \mathrm{c} 1 \mathrm{c}(\mathrm{O}) \mathrm{cc}(\mathrm{cc} 1) \mathrm{O}$ & 1.80 & $1,2,4,5,8,11,13$ \\
\hline 21 & $\mathrm{p}$ & $3360-41-6$ & 4-phenyl-1-butanol & $\mathrm{C}(\mathrm{CCCO}) \mathrm{c} 1 \mathrm{ccccc} 1$ & 0.12 & 1,2 \\
\hline 22 & $\mathrm{p}$ & $90-02-8$ & salicylaldehyde & $\mathrm{C}(=\mathrm{O}) \mathrm{c} 1 \mathrm{c}(\mathrm{O}) \mathrm{cccc} 1$ & 0.42 & $1,2,3,4,5,10,11$ \\
\hline 23 & $\mathrm{p}$ & $103-05-9$ & $\alpha, \alpha$-dimethylbenzenepropanol & $\mathrm{C}(\mathrm{CC}(\mathrm{C})(\mathrm{C}) \mathrm{O}) \mathrm{c} 1 \mathrm{ccccc} 1$ & -0.07 & $1 £ \neg 2$ \\
\hline 24 & $\mathrm{p}$ & $552-41-0$ & 2-hydroxy-4-methoxy- acetophenone & $\mathrm{C}(\mathrm{C})(=\mathrm{O}) \mathrm{c} 1 \mathrm{c}(\mathrm{O}) \mathrm{cc}(\mathrm{cc} 1) \mathrm{OC}$ & 0.55 & $1,4,5,7,8,11$ \\
\hline 25 & $\mathrm{p}$ & $22144-60-1$ & 1-phenyl-1-butanol & $\mathrm{C}([\mathrm{C} @ @ \mathrm{H}](\mathrm{O}) \mathrm{c} 1 \mathrm{ccccc} 1) \mathrm{CC}$ & -0.01 & 1 \\
\hline 26 & $\mathrm{p}$ & $103-73-1$ & ethoxybenzene & $\mathrm{O}(\mathrm{CC}) \mathrm{c} 1 \mathrm{ccccc} 1$ & 0.10 & 1,2 \\
\hline 27 & $\mathrm{p}$ & $106-48-9$ & 4-chlorophenol & Clc1ccc(cc1)O & 0.54 & $1,2,3,4,5,7,8,11,14$ \\
\hline 28 & $\mathrm{p}$ & $108-88-3$ & methylbenzene & Cc1cecc 1 & 0.25 & 1 \\
\hline 29 & $\mathrm{p}$ & $108-90-7$ & chlorobenzene & Clc1 cecce1 & -0.13 & $1,2,14$ \\
\hline 30 & $\mathrm{p}$ & $100-68-5$ & thioanisole & $\mathrm{S}(\mathrm{C}) \mathrm{c} 1 \mathrm{ccccc} 1$ & 0.18 & 1 \\
\hline 31 & $\mathrm{p}$ & $103-63-9$ & phenethyl bromide & $\mathrm{C}(\mathrm{CBr}) \mathrm{c} 1 \mathrm{ccccc} 1$ & 0.50 & 1,2 \\
\hline 32 & $\mathrm{p}$ & $10521-91-2$ & 5-phenyl-1-pentanol & $\mathrm{C}(\mathrm{CCCCO}) \mathrm{c} 1 \mathrm{ccccc} 1$ & 0.42 & 1 \\
\hline
\end{tabular}




\begin{tabular}{|c|c|c|c|c|c|c|}
\hline 33 & $\mathrm{p}$ & $59-50-7$ & 4-chloro-3-methylphenol & $\mathrm{Cc} 1 \mathrm{c}(\mathrm{Cl}) \operatorname{ccc}(\mathrm{c} 1) \mathrm{O}$ & 0.80 & $1,2,3,4,5,7,8,11,14$ \\
\hline 34 & $\mathrm{p}$ & $104-93-8$ & 4-methylanisole & $\mathrm{O}(\mathrm{C}) \mathrm{c} 1 \mathrm{ccc}(\mathrm{cc} 1) \mathrm{C}$ & 0.25 & 1 \\
\hline 35 & $\mathrm{p}$ & $134-85-0$ & 4-chlorobenzophenone & $\mathrm{C}(=\mathrm{O})(\mathrm{c} 1 \mathrm{ccc}(\mathrm{cc} 1) \mathrm{Cl}) \mathrm{c} 1 \mathrm{ccccc} 1$ & 1.50 & 1,2 \\
\hline 36 & $\mathrm{p}$ & $29338-49-6$ & 1,1-diphenyl-2-propanol & $\begin{array}{l}\mathrm{C}([\mathrm{C} @ @ \mathrm{H}](\mathrm{C}) \mathrm{O})(\mathrm{c} 1 \mathrm{cccc} 1) \mathrm{c} 1 \mathrm{cccc} \\
\mathrm{c} 1\end{array}$ & 0.75 & 1 \\
\hline 37 & $\mathrm{p}$ & $5922-60-1$ & 2-amino-5-chlorobenzonitrile & $\mathrm{C}(\# \mathrm{~N}) \mathrm{c} 1 \mathrm{c}(\mathrm{N}) \mathrm{ccc}(\mathrm{c} 1) \mathrm{Cl}$ & 0.44 & $1,2,3$ \\
\hline 38 & $\mathrm{p}$ & $3597-91-9$ & 4-biphenylmethanol & $\mathrm{C}(\mathrm{O}) \mathrm{c} 1 \mathrm{ccc}(\mathrm{cc} 1) \mathrm{c} 1 \mathrm{ccccc} 1$ & 0.92 & 1,2 \\
\hline 39 & $\mathrm{p}$ & $2973-76-4$ & 5-bromovanillin & $\mathrm{O}(\mathrm{C}) \mathrm{c} 1 \mathrm{c}(\mathrm{O}) \mathrm{c}(\mathrm{Br}) \mathrm{cc}(\mathrm{c} 1) \mathrm{C}=\mathrm{O}$ & 0.62 & $1,2,3,4,5,7,8,10,11$ \\
\hline 40 & $\mathrm{p}$ & $106-42-3$ & 1,4-dimethylbenzene & $\mathrm{Cc} 1 \mathrm{ccc}(\mathrm{cc} 1) \mathrm{C}$ & 0.25 & 1 \\
\hline 41 & $\mathrm{p}$ & $108-43-0$ & 3-chlorophenol & Clc1cc(O)ccc1 & 0.87 & $1,2,3,4,5,7,8,11$ \\
\hline 42 & $\mathrm{p}$ & $119-61-9$ & benzophenone & $\mathrm{C}(=\mathrm{O})(\mathrm{c} 1 \mathrm{ccccc} 1) \operatorname{c} 1 \mathrm{ccccc} 1$ & 0.87 & 1,2 \\
\hline 43 & $\mathrm{p}$ & $65262-96-6$ & 3-chloro-5-methoxyphenol & $\mathrm{O}(\mathrm{C}) \mathrm{c} 1 \mathrm{cc}(\mathrm{Cl}) \mathrm{cc}(\mathrm{c} 1) \mathrm{O}$ & 0.76 & $1,3,4,5,7,8,11$ \\
\hline 44 & $\mathrm{p}$ & $5342-87-0$ & $( \pm)$-1,2-diphenyl-2-propanol & $\mathrm{C}([\mathrm{C} @](\mathrm{C})(\mathrm{O}) \mathrm{c} 1 \mathrm{ccc} c \mathrm{1}) \mathrm{c} 1 \mathrm{cccc} 1$ & 0.80 & 1 \\
\hline 45 & $\mathrm{p}$ & $554-00-7$ & 3,4-dichloroaniline & $\mathrm{Clc1c}(\mathrm{N}) \operatorname{ccc}(\mathrm{c} 1) \mathrm{Cl}$ & 0.56 & 1,2 \\
\hline 46 & $\mathrm{p}$ & $2430-16-2$ & 6-phenyl-1-hexanol & $\mathrm{C}(\mathrm{CCCCCO}) \mathrm{c} 1 \mathrm{ccccc} 1$ & 0.87 & 1 \\
\hline 47 & $\mathrm{p}$ & $95-82-9$ & 2,5-dichloroaniline & $\mathrm{Nc1c}(\mathrm{Cl}) \operatorname{ccc}(\mathrm{c} 1) \mathrm{Cl}$ & 0.58 & 1,2 \\
\hline 48 & $\mathrm{p}$ & $98-82-8$ & isopropylbenzene & $\mathrm{C}(\mathrm{C})(\mathrm{C}) \mathrm{c} 1 \mathrm{ccccc} 1$ & 0.69 & $1,2,14$ \\
\hline 49 & $\mathrm{p}$ & $626-43-7$ & 3,5-dichloroaniine & $\mathrm{Clc} 1 \mathrm{cc}(\mathrm{Cl}) \mathrm{cc}(\mathrm{c} 1) \mathrm{N}$ & 0.71 & 1,2 \\
\hline 50 & $\mathrm{p}$ & $92-52-4$ & biphenyl & $\mathrm{c} 1(\operatorname{ccccc} 1) \mathrm{c} 1 \operatorname{ccccc} 1$ & 1.05 & $1,2,14$ \\
\hline 51 & $\mathrm{p}$ & $108-86-1$ & bromobenzene & Brcleccec1 & 0.75 & $1,2,14$ \\
\hline 52 & $\mathrm{p}$ & $104-51-8$ & $n$-butylbenzene & $\mathrm{C}(\mathrm{CCC}) \mathrm{c} 1 \mathrm{ccccc} 1$ & 1.25 & $1,2,14$ \\
\hline 53 & $\mathrm{p}$ & $120-83-2$ & 2,4-dichlorophenol & $\mathrm{Clc} 1 \mathrm{c}(\mathrm{O}) \operatorname{ccc}(\mathrm{c} 1) \mathrm{Cl}$ & 1.04 & $1,2,3,4,5,7,8,11,14$ \\
\hline 54 & $\mathrm{p}$ & $538-68-1$ & $n$-amylbenzene & $\mathrm{C}(\mathrm{CCCC}) \mathrm{c} 1 \mathrm{ccccc} 1$ & 1.79 & $1,2,14$ \\
\hline 55 & $\mathrm{p}$ & $88-04-0$ & 4-chloro-3,5-dimethylphenol & $\mathrm{Clclc}(\mathrm{C}) \operatorname{cc}(\operatorname{cc} 1 \mathrm{C}) \mathrm{O}$ & 1.20 & $1,2,3,4,5,7,8,11$ \\
\hline 56 & $\mathrm{p}$ & $5707-44-8$ & 4-ethylbiphenyl & $\mathrm{C}(\mathrm{C}) \mathrm{c} 1 \mathrm{ccc}(\mathrm{cc} 1) \mathrm{c} 1 \mathrm{ccccc} 1$ & 1.97 & 1 \\
\hline 57 & $\mathrm{p}$ & $106-38-7$ & 4-bromotoluene & Brc1ccc(cc1)C & 1.00 & 1 \\
\hline 58 & $\mathrm{p}$ & $62-53-3$ & aniline & Nc1eccec1 & -0.23 & $1,2,14$ \\
\hline 59 & $\mathrm{p}$ & $95-75-0$ & 3,4-dichlorotoluene & $\mathrm{Clc1c}(\mathrm{Cl}) \operatorname{ccc}(\mathrm{c} 1) \mathrm{C}$ & 1.07 & $1,2,14$ \\
\hline 60 & $\mathrm{p}$ & $106-49-0$ & 4-methylaniline & Cc1cce $(\operatorname{cc} 1) \mathrm{N}$ & -0.05 & $1,2,14$ \\
\hline 61 & $\mathrm{p}$ & $1585-07-5$ & 1-bromo-4-ethylbenzene & $\mathrm{C}(\mathrm{C}) \mathrm{c} 1 \mathrm{ccc}(\mathrm{cc} 1) \mathrm{Br}$ & 1.10 & 1 \\
\hline 62 & $\mathrm{p}$ & $108-44-1$ & 3-methylaniline & $\mathrm{Cc} 1 \mathrm{cc}(\mathrm{N}) \operatorname{ccc} 1$ & -0.28 & $1,2,3$ \\
\hline 63 & $\mathrm{p}$ & $99-09-2$ & 3-nitroaniline & $\mathrm{N}(=\mathrm{O})(=\mathrm{O}) \mathrm{c} 1 \mathrm{cc}(\mathrm{N}) \operatorname{ccc} 1$ & 0.03 & 1,3 \\
\hline 64 & $\mathrm{p}$ & $95-53-4$ & 2-methylaniline & $\mathrm{Cc} 1 \mathrm{c}(\mathrm{N}) \operatorname{cccc} 1$ & -0.16 & 1,2 \\
\hline 65 & $\mathrm{p}$ & $98-95-3$ & nitrobenzene & $\mathrm{N}(=\mathrm{O})(=\mathrm{O}) \mathrm{c} 1 \mathrm{ccccc} 1$ & 0.14 & $1,2,3,14$ \\
\hline 66 & $\mathrm{p}$ & $578-54-1$ & 2-ethylaniline & $\mathrm{C}(\mathrm{C}) \mathrm{clc}(\mathrm{N}) \operatorname{cccc} 1$ & -0.22 & 1,2 \\
\hline 67 & $\mathrm{p}$ & $555-03-3$ & 3-nitroanisole & $\mathrm{N}(=\mathrm{O})(=\mathrm{O}) \mathrm{c} 1 \mathrm{cc}(\mathrm{OC}) \mathrm{ccc} 1$ & 0.72 & $1,2,3$ \\
\hline 68 & $\mathrm{p}$ & $127-66-2$ & 2-phenyl-3-butyn-2-ol & $\mathrm{C}([\mathrm{C} @](\mathrm{C})(\mathrm{O}) \mathrm{c} 1 \mathrm{ccccc} 1) \# \mathrm{C}$ & -0.18 & $1,6,12$ \\
\hline 69 & $\mathrm{p}$ & $88-72-2$ & 2-nitrotoluene & $\mathrm{N}(=\mathrm{O})(=\mathrm{O}) \mathrm{clc}(\mathrm{C}) \mathrm{cccc} 1$ & 0.26 & $1,2,3$ \\
\hline 70 & $\mathrm{p}$ & $587-02-0$ & 3-ethylaniline & $\mathrm{C}(\mathrm{C}) \mathrm{clcc}(\mathrm{N}) \mathrm{ccc} 1$ & -0.03 & 1,2 \\
\hline
\end{tabular}




\begin{tabular}{|c|c|c|c|c|c|c|}
\hline 71 & $\mathrm{p}$ & $99-99-0$ & 4-nitrotoluene & $\mathrm{N}(=\mathrm{O})(=\mathrm{O}) \mathrm{c} 1 \mathrm{ccc}(\mathrm{cc} 1) \mathrm{C}$ & 0.65 & $1,2,3$ \\
\hline 72 & $\mathrm{p}$ & $589-16-2$ & 4-ethylaniline & $\mathrm{C}(\mathrm{C}) \mathrm{c} 1 \operatorname{ccc}(\operatorname{cc} 1) \mathrm{N}$ & 0.03 & $1,2,14$ \\
\hline 73 & $\mathrm{p}$ & $616-86-4$ & 4-ethoxy-2-nitroaniline & $\mathrm{N}(=\mathrm{O})(=\mathrm{O}) \mathrm{c} 1 \mathrm{cc}(\mathrm{OCC}) \operatorname{ccc} 1 \mathrm{~N}$ & 0.76 & $1,2,14$ \\
\hline 74 & $\mathrm{p}$ & $4344-55-2$ & 4-butoxyaniline & $\mathrm{O}(\mathrm{CCCC}) \mathrm{c} 1 \mathrm{ccc}(\mathrm{cc} 1) \mathrm{N}$ & 0.61 & 1,2 \\
\hline 75 & $\mathrm{p}$ & $99-08-1$ & 3-nitrotoluene & $\mathrm{N}(=\mathrm{O})(=\mathrm{O}) \mathrm{c} 1 \mathrm{cc}(\mathrm{C}) \mathrm{ccc} 1$ & 0.42 & $1,2,3,14$ \\
\hline 76 & $\mathrm{p}$ & $579-66-8$ & 2,6-diethylaniline & $\mathrm{C}(\mathrm{C}) \mathrm{c} 1 \mathrm{c}(\mathrm{N}) \mathrm{c}(\mathrm{CC}) \mathrm{ccc} 1$ & 0.31 & 1,2 \\
\hline 77 & $\mathrm{p}$ & $2357-47-3$ & $\alpha, \alpha, \alpha$-4-tetrafluoro- $m$-toluidine & $\mathrm{C}(\mathrm{F})(\mathrm{F})(\mathrm{F}) \mathrm{c} 1 \mathrm{c}(\mathrm{F}) \operatorname{ccc}(\mathrm{c} 1) \mathrm{N}$ & 0.77 & $1,2,14$ \\
\hline 78 & $\mathrm{p}$ & $39905-50-5$ & 4-pentyloxyaniline & $\mathrm{O}(\mathrm{CCCCC}) \mathrm{c} 1 \mathrm{ccc}(\mathrm{cc} 1) \mathrm{N}$ & 0.97 & 1 \\
\hline 79 & $\mathrm{p}$ & $100-29-8$ & 4-nitrophenetole & $\mathrm{N}(=\mathrm{O})(=\mathrm{O}) \mathrm{c} 1 \mathrm{ccc}(\mathrm{cc} 1) \mathrm{OCC}$ & 0.83 & 1,2 \\
\hline 80 & $\mathrm{p}$ & $24544-04-5$ & 2,6-diisopropylaniline & $\mathrm{C}(\mathrm{C})(\mathrm{C}) \mathrm{c} 1 \mathrm{c}(\mathrm{N}) \mathrm{c}(\mathrm{C}(\mathrm{C}) \mathrm{C}) \mathrm{ccc} 1$ & 0.76 & 1,2 \\
\hline 81 & $\mathrm{p}$ & $83-41-0$ & 1,2-dimethyl-3-nitrobenzene & $\mathrm{N}(=\mathrm{O})(=\mathrm{O}) \mathrm{c} 1 \mathrm{c}(\mathrm{C}) \mathrm{c}(\mathrm{C}) \mathrm{ccc} 1$ & 0.56 & 1 \\
\hline 82 & $\mathrm{p}$ & $104-13-2$ & 4-butylaniline & $\mathrm{C}(\mathrm{CCC}) \mathrm{c} 1 \operatorname{ccc}(\mathrm{cc} 1) \mathrm{N}$ & 1.07 & $1,2,14$ \\
\hline 83 & $\mathrm{p}$ & $99-51-4$ & 1,2-dimethyl-4-nitrobenzene & $\mathrm{N}(=\mathrm{O})(=\mathrm{O}) \mathrm{c} 1 \mathrm{cc}(\mathrm{C}) \mathrm{c}(\mathrm{cc} 1) \mathrm{C}$ & 0.59 & 1,2 \\
\hline 84 & $\mathrm{p}$ & $150-76-5$ & 4-methoxyphenol & $\mathrm{O}(\mathrm{C}) \mathrm{c} 1 \mathrm{ccc}(\mathrm{cc} 1) \mathrm{O}$ & -0.14 & $1,2,3,4,5,7,8,11,14$ \\
\hline 85 & $\mathrm{p}$ & $95-50-1$ & 1,2-dichlorobenzene & Clc1c(Cl)ccc1 & 1.00 & $1,3,14$ \\
\hline 86 & $\mathrm{p}$ & $100-52-7$ & benzaldehyde & $\mathrm{C}(=\mathrm{O}) \mathrm{c} 1 \mathrm{ccccc} 1$ & -0.20 & $1,2,10$ \\
\hline 87 & $\mathrm{p}$ & $591-35-5$ & 3,5-dichlorophenol & $\mathrm{Clc1cc}(\mathrm{Cl}) \mathrm{cc}(\mathrm{c} 1) \mathrm{O}$ & 1.56 & $1,2,3,4,5,7,8,11$ \\
\hline 88 & $\mathrm{p}$ & $108-95-2$ & phenol & Oc1ccect 1 & -0.35 & $1,2,3,4,5,7,8,11,14$ \\
\hline 89 & $\mathrm{p}$ & $636-30-6$ & 2,4,5-trichloroaniline & $\mathrm{Clc1c}(\mathrm{Cl}) \operatorname{cc}(\mathrm{c}(\mathrm{c} 1) \mathrm{Cl}) \mathrm{N}$ & 1.30 & 1,2 \\
\hline 90 & $\mathrm{p}$ & $150-19-6$ & 3-methoxyphenol & $\mathrm{O}(\mathrm{C}) \mathrm{c} 1 \mathrm{cc}(\mathrm{O}) \mathrm{ccc} 1$ & -0.33 & $1,2,3,4,5,7,8,11,14$ \\
\hline 91 & $\mathrm{p}$ & $106-37-6$ & 1,4-dibromobenzene & $\operatorname{Brclccc}(\operatorname{cc} 1) \mathrm{Br}$ & 0.68 & 1 \\
\hline 92 & $\mathrm{p}$ & $121-32-4$ & 3-ethoxy-4-hydroxybenzaldehyde & $\mathrm{O}(\mathrm{CC}) \mathrm{c} 1 \mathrm{cc}(\mathrm{C}=\mathrm{O}) \operatorname{ccc} 1 \mathrm{O}$ & 0.02 & $1,3,4,5,7,8,10,11$ \\
\hline 93 & $\mathrm{p}$ & $98-86-2$ & acetophenone & $\mathrm{C}(\mathrm{C})(=\mathrm{O}) \mathrm{c} 1 \mathrm{ccccc} 1$ & -0.05 & 1,2 \\
\hline 94 & $\mathrm{p}$ & $88-75-5$ & 2-nitrophenol & $\mathrm{N}(=\mathrm{O})(=\mathrm{O}) \mathrm{c} 1 \mathrm{c}(\mathrm{O}) \mathrm{cccc} 1$ & 0.67 & $1,2,3,4,5,7,8,1620$ \\
\hline 95 & $\mathrm{p}$ & $106-44-5$ & 4-methylphenol & $\mathrm{Cc} 1 \mathrm{ccc}(\mathrm{cc} 1) \mathrm{O}$ & -0.16 & $1,2,4,8,14$ \\
\hline 96 & $\mathrm{p}$ & $88-74-4$ & 2-nitroaniline & $\mathrm{N}(=\mathrm{O})(=\mathrm{O}) \mathrm{c} 1 \mathrm{c}(\mathrm{N}) \operatorname{ccc} 1$ & 0.08 & $1,2,3$ \\
\hline 97 & $\mathrm{p}$ & $95-48-7$ & 2-methylphenol & $\mathrm{Cc1c}(\mathrm{O}) \mathrm{cccc} 1$ & -0.29 & $1,4,14$ \\
\hline 98 & $\mathrm{p}$ & $121-87-9$ & 2-chloro-4-nitroaniline & $\mathrm{N}(=\mathrm{O})(=\mathrm{O}) \mathrm{c} 1 \mathrm{cc}(\mathrm{Cl}) \mathrm{c}(\mathrm{cc} 1) \mathrm{N}$ & 0.75 & 1,2 \\
\hline 99 & $\mathrm{p}$ & $108-39-4$ & 3-methylphenol & $\mathrm{Cc} 1 \mathrm{cc}(\mathrm{O}) \mathrm{ccc} 1$ & -0.08 & $1,2,3,4,8$ \\
\hline 100 & $\mathrm{p}$ & $104-88-1$ & 4-chlorobenzaldehyde & $\mathrm{C}(=\mathrm{O}) \mathrm{c} 1 \mathrm{ccc}(\mathrm{cc} 1) \mathrm{Cl}$ & 0.40 & $1,2,10$ \\
\hline 101 & $\mathrm{p}$ & $93-55-0$ & propiophenone & $\mathrm{C}(\mathrm{CC})(=\mathrm{O}) \mathrm{c} 1 \mathrm{ccccc} 1$ & 0.05 & 1,2 \\
\hline 102 & $\mathrm{p}$ & $653-37-2$ & pentafluorobenzaldehyde & $\mathrm{C}(=\mathrm{O}) \mathrm{c} 1 \mathrm{c}(\mathrm{F}) \mathrm{c}(\mathrm{F}) \mathrm{c}(\mathrm{c}(\mathrm{c} 1 \mathrm{~F}) \mathrm{F}) \mathrm{F}$ & 0.82 & $1,2,10$ \\
\hline 103 & $\mathrm{p}$ & $105-67-9$ & 2,4-dimethylphenol & $\mathrm{Cc} 1 \mathrm{c}(\mathrm{O}) \operatorname{ccc}(\mathrm{c} 1) \mathrm{C}$ & 0.14 & $1,3,4,5,7,8,11,14$ \\
\hline 104 & $\mathrm{p}$ & $88-06-2$ & 2,4,6-trichlorophenol & $\mathrm{Oc} 1 \mathrm{c}(\mathrm{Cl}) \mathrm{cc}(\mathrm{cc} 1 \mathrm{Cl}) \mathrm{Cl}$ & 1.41 & $1,2,3,4,5,7,8,11,14$ \\
\hline 105 & $\mathrm{p}$ & $90-00-6$ & 2-ethylphenol & $\mathrm{C}(\mathrm{C}) \mathrm{c} 1 \mathrm{c}(\mathrm{O}) \mathrm{cccc} 1$ & 0.16 & $1,3,4,5,7,8,11$ \\
\hline 106 & $\mathrm{p}$ & $95-95-4$ & 2,4,5-trichlorophenol & $\mathrm{Clc1c}(\mathrm{Cl}) \mathrm{cc}(\mathrm{c}(\mathrm{c} 1) \mathrm{Cl}) \mathrm{O}$ & 2.10 & $1,2,3,4,5,7,8,11$ \\
\hline 107 & $\mathrm{p}$ & $123-07-9$ & 4-ethylphenol & $\mathrm{C}(\mathrm{C}) \mathrm{c} 1 \mathrm{ccc}(\mathrm{cc} 1) \mathrm{O}$ & 0.21 & $1,2,3,4,5,7,8,11,14$ \\
\hline 108 & $\mathrm{p}$ & $120-82-1$ & 1,2,4-trichlorobenzene & $\mathrm{Clclc}(\mathrm{Cl}) \operatorname{ccc}(\mathrm{c} 1) \mathrm{Cl}$ & 1.10 & $1,2,14$ \\
\hline 109 & $\mathrm{p}$ & $620-17-7$ & 3-ethylphenol & $\mathrm{C}(\mathrm{C}) \mathrm{c} 1 \mathrm{cc}(\mathrm{O}) \mathrm{ccc} 1$ & 0.29 & $1,2,3,4,11$ \\
\hline
\end{tabular}




\begin{tabular}{|c|c|c|c|c|c|c|}
\hline 110 & $\mathrm{p}$ & $118-79-6$ & 2,4,6-tribromophenol & $\mathrm{Oc} 1 \mathrm{c}(\mathrm{Br}) \mathrm{cc}(\mathrm{cc} 1 \mathrm{Br}) \mathrm{Br}$ & 1.91 & $1,2,3,4,5,7,8,11,14$ \\
\hline 111 & $\mathrm{p}$ & $1745-81-9$ & 2-allylphenol & $\mathrm{C}(\mathrm{C}=\mathrm{C}) \mathrm{c} 1 \mathrm{c}(\mathrm{O}) \operatorname{ccc} 1$ & 0.33 & $1,2,3,4,5,7,8,11,14$ \\
\hline 112 & $\mathrm{p}$ & $108-70-3$ & 1,3,5-trichlorobenzene & $\mathrm{Clc1cc}(\mathrm{Cl}) \operatorname{cc}(\mathrm{c} 1) \mathrm{Cl}$ & 0.87 & 1 \\
\hline 113 & $\mathrm{p}$ & $2416-94-6$ & 2,3,6-trimethylphenol & $\mathrm{Cc} 1 \mathrm{c}(\mathrm{O}) \mathrm{c}(\mathrm{C}) \mathrm{ccc} 1 \mathrm{C}$ & 0.28 & $1,3,4,5,7,11,14$ \\
\hline 114 & $\mathrm{p}$ & $771-60-8$ & pentafluoroaniline & $\mathrm{Fclc}(\mathrm{F}) \mathrm{c}(\mathrm{F}) \mathrm{c}(\mathrm{c}(\mathrm{c} 1 \mathrm{~F}) \mathrm{F}) \mathrm{N}$ & 0.26 & $1,2,14$ \\
\hline 115 & $\mathrm{p}$ & $527-60-6$ & 2,4,6-trimethylphenol & Oc1c(C)cc(cc1C)C & 0.42 & $1,2,3,4,5,7,8,11,14$ \\
\hline 116 & $\mathrm{p}$ & $350-46-9$ & 1-fluoro-4-nitrobenzene & $\mathrm{N}(=\mathrm{O})(=\mathrm{O}) \mathrm{c} 1 \operatorname{ccc}(\operatorname{cc} 1) \mathrm{F}$ & 0.10 & $1,2,14$ \\
\hline 117 & $\mathrm{p}$ & $495-40-9$ & butyrophenone & $\mathrm{C}(\mathrm{CCC})(=\mathrm{O}) \mathrm{c} 1 \operatorname{ccccc} 1$ & 0.30 & 1,2 \\
\hline 118 & $\mathrm{p}$ & $121-73-3$ & 1-chloro-3-nitrobenzene & $\mathrm{N}(=\mathrm{O})(=\mathrm{O}) \mathrm{clcc}(\mathrm{Cl}) \operatorname{ccc} 1$ & 0.73 & $1,2,14$ \\
\hline 119 & $\mathrm{p}$ & $527-54-8$ & 3,4,5-trimethylphenol & $\mathrm{Cc} 1 \mathrm{c}(\mathrm{C}) \mathrm{cc}(\mathrm{cc} 1 \mathrm{C}) \mathrm{O}$ & 0.93 & $1,2,3,4,5,7,8,11$ \\
\hline 120 & $\mathrm{p}$ & $89-59-8$ & 4-chloro-2-nitrotoluene & $\mathrm{N}(=\mathrm{O})(=\mathrm{O}) \mathrm{clc}(\mathrm{C}) \operatorname{ccc}(\mathrm{c} 1) \mathrm{Cl}$ & 0.82 & $1,2,3$ \\
\hline 121 & $\mathrm{p}$ & $697-82-5$ & 2,3,5-trimethylphenol & $\mathrm{Cc} 1 \mathrm{c}(\mathrm{C}) \operatorname{cc}(\mathrm{cc} 1 \mathrm{O}) \mathrm{C}$ & 0.36 & $1,2,3,4,5,7,8,11$ \\
\hline 122 & $\mathrm{p}$ & $83-42-1$ & 2-chloro-6-nitrotoluene & $\mathrm{N}(=\mathrm{O})(=\mathrm{O}) \mathrm{c} 1 \mathrm{c}(\mathrm{C}) \mathrm{c}(\mathrm{Cl}) \mathrm{ccc} 1$ & 0.68 & $1,2,3$ \\
\hline 123 & $\mathrm{p}$ & $122-03-2$ & 4-isopropylbenzaldehyde & $\mathrm{C}(\mathrm{C})(\mathrm{C}) \mathrm{c} 1 \mathrm{ccc}(\mathrm{cc} 1) \mathrm{C}=\mathrm{O}$ & 0.67 & 1,10 \\
\hline 124 & $\mathrm{p}$ & $3481-20-7$ & 2,3,5,6-tetrachloroaniline & $\mathrm{Clclc}(\mathrm{N}) \mathrm{c}(\mathrm{Cl}) \mathrm{c}(\mathrm{cclCl}) \mathrm{Cl}$ & 1.76 & 1,14 \\
\hline 125 & $\mathrm{p}$ & $1009-14-9$ & valerophenone & $\mathrm{C}(\mathrm{CCCC})(=\mathrm{O}) \mathrm{c} 1 \mathrm{ccccc} 1$ & 0.56 & 1,2 \\
\hline 126 & $\mathrm{p}$ & $634-83-3$ & 2,3,4,5-tetrachloroaniline & $\mathrm{Clclc}(\mathrm{Cl}) \mathrm{c}(\mathrm{Cl}) \mathrm{c}(\mathrm{cclCl}) \mathrm{N}$ & 1.96 & 1,2 \\
\hline 127 & $\mathrm{p}$ & $645-56-7$ & 4-propylphenol & $\mathrm{C}(\mathrm{CC}) \mathrm{c} 1 \mathrm{ccc}(\mathrm{cc} 1) \mathrm{O}$ & 0.64 & $1,2,3,4,5,7,8,11,14$ \\
\hline 128 & $\mathrm{p}$ & $95-94-3$ & 1,2,4,5-tetrachlorobenzene & $\mathrm{Clclc}(\mathrm{Cl}) \mathrm{cc}(\mathrm{c}(\mathrm{c} 1) \mathrm{Cl}) \mathrm{Cl}$ & 2.00 & 1 \\
\hline 129 & $\mathrm{p}$ & $591-50-4$ & iodobenzene & Ic1 $1 \mathrm{cccc} 1$ & 0.50 & 1,2 \\
\hline 130 & $\mathrm{p}$ & $6641-64-1$ & 4,5-dichloro-2-nitroaniline & $\mathrm{N}(=\mathrm{O})(=\mathrm{O}) \mathrm{c} 1 \mathrm{c}(\mathrm{N}) \mathrm{cc}(\mathrm{c}(\mathrm{c} 1) \mathrm{Cl}) \mathrm{Cl}$ & 1.66 & 1,2 \\
\hline 131 & $\mathrm{p}$ & $98-54-4$ & 4-tert-butylphenol & $\mathrm{C}(\mathrm{C})(\mathrm{C})(\mathrm{C}) \mathrm{c} 1 \mathrm{ccc}(\mathrm{cc} 1) \mathrm{O}$ & 0.91 & $1,2,3,4,8,14$ \\
\hline 132 & $\mathrm{p}$ & $103-72-0$ & phenyl isothiocyanate & $\mathrm{N}(=\mathrm{C}=\mathrm{S}) \mathrm{c} 1 \operatorname{ccccc} 1$ & 1.41 & 1 \\
\hline 133 & $\mathrm{p}$ & $39905-57-2$ & 4-hexyloxyaniline & $\mathrm{O}(\mathrm{CCCCCC}) \mathrm{c} 1 \mathrm{ccc}(\mathrm{cc} 1) \mathrm{N}$ & 1.38 & $1,2,14$ \\
\hline 134 & $\mathrm{p}$ & $2683-43-4$ & 2,4-dichloro-6-nitroaniline & $\mathrm{N}(=\mathrm{O})(=\mathrm{O}) \mathrm{c} 1 \mathrm{c}(\mathrm{N}) \mathrm{c}(\mathrm{Cl}) \mathrm{cc}(\mathrm{c} 1) \mathrm{Cl}$ & 1.26 & 1,2 \\
\hline 135 & $\mathrm{p}$ & $80-46-6$ & 4-tert-pentylphenol & $\mathrm{C}(\mathrm{CC})(\mathrm{C})(\mathrm{C}) \mathrm{c} 1 \mathrm{ccc}(\mathrm{cc} 1) \mathrm{O}$ & 1.23 & $1,2,3,4,8,14$ \\
\hline 136 & $\mathrm{p}$ & $90-59-5$ & 3,5-dibromosalicylaldehyde & $\mathrm{C}(=\mathrm{O}) \mathrm{c} 1 \mathrm{c}(\mathrm{O}) \mathrm{c}(\mathrm{Br}) \mathrm{cc}(\mathrm{c} 1) \mathrm{Br}$ & 1.65 & $1,3,4,5,7,8,10,11$ \\
\hline 137 & $\mathrm{p}$ & $5736-91-4$ & 4-pentyloxybenzaldehyde & $\mathrm{O}(\mathrm{CCCCC}) \mathrm{c} 1 \operatorname{ccc}(\operatorname{cc} 1) \mathrm{C}=\mathrm{O}$ & 1.18 & 1,10 \\
\hline 138 & $\mathrm{p}$ & $58-90-2$ & 2,3,4,6-tetrachlorophenol & $\mathrm{Clclc}(\mathrm{Cl}) \mathrm{c}(\mathrm{O}) \mathrm{c}(\mathrm{cclCl}) \mathrm{Cl}$ & 2.18 & 1,2 \\
\hline 139 & $\mathrm{p}$ & $1671-75-6$ & heptanophenone & $\mathrm{C}(\mathrm{CCCCCC})(=\mathrm{O}) \mathrm{c} 1 \mathrm{ccccc} 1$ & 1.56 & 1,2 \\
\hline 140 & $\mathrm{p}$ & $4901-51-3$ & 2,3,4,5-tetrachlorophenol & $\mathrm{Clc1c}(\mathrm{Cl}) \mathrm{c}(\mathrm{Cl}) \mathrm{c}(\mathrm{cc} 1 \mathrm{Cl}) \mathrm{O}$ & 2.72 & $1,3,4,5,7,8,11,14$ \\
\hline 141 & $\mathrm{p}$ & $1674-37-9$ & octanophenone & $\mathrm{C}(\mathrm{CCCCCCC})(=\mathrm{O}) \mathrm{c} 1 \mathrm{ccccc} 1$ & 1.89 & 1,2 \\
\hline 142 & $\mathrm{p}$ & $104-40-5$ & nonylphenol & $\mathrm{C}(\mathrm{CCCCCCCC}) \mathrm{c} 1 \mathrm{ccc}(\mathrm{cc} 1) \mathrm{O}$ & 2.47 & $1,2,3,4,5,8,11,14$ \\
\hline 143 & $\mathrm{n}$ & $108-46-3$ & 1,3-dihydroxybenzene & Oc1cc(O)ccc1 & -0.65 & $1,2,4,5,7,8,11,13,14$ \\
\hline 144 & $\mathrm{p}$ & $2227-79-4$ & thiobenzamide & $\mathrm{C}(\mathrm{N})(=\mathrm{S}) \mathrm{c} 1 \mathrm{ccccc} 1$ & 0.09 & 1 \\
\hline 145 & $\mathrm{p}$ & $6361-21-3$ & 2-chloro-5-nitrobenzaldehyde & $\mathrm{C}(=\mathrm{O}) \mathrm{c} 1 \mathrm{cc}(\mathrm{N}(=\mathrm{O})=\mathrm{O}) \operatorname{ccc} 1 \mathrm{Cl}$ & 0.53 & $1,2,10$ \\
\hline 146 & $\mathrm{p}$ & $100-00-5$ & 1-chloro-4-nitrobenzene & $\mathrm{N}(=\mathrm{O})(=\mathrm{O}) \mathrm{c} 1 \mathrm{ccc}(\mathrm{cc} 1) \mathrm{Cl}$ & 0.43 & 1,2 \\
\hline 147 & $\mathrm{p}$ & $3531-19-9$ & 6-chloro-2,4-dinitroaniline & $\begin{array}{l}\mathrm{N}(=\mathrm{O})(=\mathrm{O}) \mathrm{c} 1 \mathrm{cc}(\mathrm{N}(=\mathrm{O})=\mathrm{O}) \operatorname{cc}(\mathrm{c} 1 \mathrm{~N}) \\
\mathrm{Cl}\end{array}$ & 1.12 & 1 \\
\hline
\end{tabular}




\begin{tabular}{|c|c|c|c|c|c|c|}
\hline 148 & $\mathrm{p}$ & $610-78-6$ & 4-chloro-3-nitrophenol & $\mathrm{N}(=\mathrm{O})(=\mathrm{O}) \mathrm{c} 1 \mathrm{c}(\mathrm{Cl}) \operatorname{ccc}(\mathrm{c} 1) \mathrm{O}$ & 1.27 & 1 \\
\hline 149 & $\mathrm{p}$ & $1817-73-8$ & 2-bromo-4,6-dinitroaniline & $\begin{array}{l}\mathrm{N}(=\mathrm{O})(=\mathrm{O}) \mathrm{c} 1 \mathrm{cc}(\mathrm{N}(=\mathrm{O})=\mathrm{O}) \operatorname{cc}(\mathrm{c} 1 \mathrm{~N}) \\
\mathrm{Br}\end{array}$ & 1.24 & 1 \\
\hline 150 & $\mathrm{p}$ & $577-19-5$ & 1-bromo-2-nitrobenzene & $\mathrm{N}(=\mathrm{O})(=\mathrm{O}) \mathrm{clc}(\mathrm{Br}) \operatorname{cccc} 1$ & 0.75 & 1,2 \\
\hline 151 & $\mathrm{p}$ & $89-69-0$ & 1,2,4-trichloro-5-nitrobenzene & $\mathrm{N}(=\mathrm{O})(=\mathrm{O}) \mathrm{c} 1 \mathrm{c}(\mathrm{Cl}) \mathrm{cc}(\mathrm{c}(\mathrm{cl}) \mathrm{Cl}) \mathrm{Cl}$ & 1.53 & 1,2 \\
\hline 152 & $\mathrm{p}$ & $88-73-3$ & 1-chloro-2-nitrobenzene & $\mathrm{N}(=\mathrm{O})(=\mathrm{O}) \mathrm{clc}(\mathrm{Cl}) \operatorname{cccc} 1$ & 0.68 & $1,2,14$ \\
\hline 153 & $\mathrm{p}$ & $17700-09-3$ & 1,2,3-trichloro-4-nitrobenzene & $\mathrm{N}(=\mathrm{O})(=\mathrm{O}) \mathrm{clc}(\mathrm{Cl}) \mathrm{c}(\mathrm{Cl}) \mathrm{c}(\mathrm{cc} 1) \mathrm{Cl}$ & 1.51 & 1 \\
\hline 154 & $\mathrm{p}$ & $585-79-5$ & 1-bromo-3-nitrobenzene & $\mathrm{N}(=\mathrm{O})(=\mathrm{O}) \mathrm{c} 1 \mathrm{cc}(\mathrm{Br}) \mathrm{ccc} 1$ & 1.03 & 1,2 \\
\hline 155 & $\mathrm{p}$ & $18708-70-8$ & 1,3,5-trichloro-2-nitrobenzene & $\mathrm{N}(=\mathrm{O})(=\mathrm{O}) \mathrm{c} 1 \mathrm{c}(\mathrm{Cl}) \mathrm{cc}(\mathrm{cc} 1 \mathrm{Cl}) \mathrm{Cl}$ & 1.43 & 1 \\
\hline 156 & $\mathrm{p}$ & $88-30-2$ & 3-(trifluoromethyl)-4-nitrophenol & $\mathrm{C}(\mathrm{F})(\mathrm{F})(\mathrm{F}) \mathrm{c} 1 \mathrm{c}(\mathrm{N}(=\mathrm{O})=\mathrm{O}) \operatorname{ccc}(\mathrm{c} 1) \mathrm{O}$ & 1.65 & 1,4 \\
\hline 157 & $\mathrm{p}$ & $573-56-8$ & 2,6-dinitrophenol & $\mathrm{N}(=\mathrm{O})(=\mathrm{O}) \mathrm{c} 1 \mathrm{c}(\mathrm{O}) \mathrm{c}(\mathrm{N}(=\mathrm{O})=\mathrm{O}) \mathrm{ccc} 1$ & 0.83 & $1,2,3,4,5,7,8,11,14$ \\
\hline 158 & $\mathrm{p}$ & $3819-88-3$ & 1-fluoro-3-iodo-5-nitrobenzene & $\mathrm{N}(=\mathrm{O})(=\mathrm{O}) \mathrm{c} 1 \mathrm{cc}(\mathrm{F}) \mathrm{cc}(\mathrm{c} 1) \mathrm{I}$ & 1.09 & 1 \\
\hline 159 & $\mathrm{p}$ & $51-28-5$ & 2,4-dinitrophenol & $\mathrm{N}(=\mathrm{O})(=\mathrm{O}) \mathrm{c} 1 \mathrm{cc}(\mathrm{N}(=\mathrm{O})=\mathrm{O}) \operatorname{ccc} 1 \mathrm{O}$ & 1.06 & $1,2,3,4,5,7,8,11,14$ \\
\hline 160 & $\mathrm{p}$ & $7149-70-4$ & 2-bromo-1-methyl-5-nitrobenzene & $\mathrm{N}(=\mathrm{O})(=\mathrm{O}) \mathrm{c} 1 \mathrm{cc}(\mathrm{C}) \mathrm{c}(\mathrm{cc} 1) \mathrm{Br}$ & 1.16 & 1 \\
\hline 161 & $\mathrm{p}$ & $329-71-5$ & 2,5-dinitrophenol & $\mathrm{N}(=\mathrm{O})(=\mathrm{O}) \mathrm{c} 1 \mathrm{cc}(\mathrm{O}) \mathrm{c}(\mathrm{cc} 1) \mathrm{N}(=\mathrm{O})=\mathrm{O}$ & 1.04 & $1,2,3,4,5,7,8,11,14$ \\
\hline 162 & $\mathrm{p}$ & $576-55-6$ & 3,4,5,6-tetrabromo-2-methylphenol & $\mathrm{Brclc}(\mathrm{Br}) \mathrm{c}(\mathrm{Br}) \mathrm{c}(\mathrm{c}(\mathrm{c} 1 \mathrm{Br}) \mathrm{C}) \mathrm{O}$ & 2.57 & $1,3,4,5,7,8,11$ \\
\hline 163 & $\mathrm{p}$ & $534-52-1$ & 4,6-dinitro-2-methylphenol & $\begin{array}{l}\mathrm{N}(=\mathrm{O})(=\mathrm{O}) \mathrm{c} 1 \mathrm{cc}(\mathrm{N}(=\mathrm{O})=\mathrm{O}) \operatorname{cc}(\mathrm{c} 1 \mathrm{O}) \\
\mathrm{C}\end{array}$ & 1.73 & $1,2,4,14$ \\
\hline 164 & $\mathrm{p}$ & $4097-49-8$ & 4-tert-butyl-2,6-dinitrophenol & $\begin{array}{l}\mathrm{N}(=\mathrm{O})(=\mathrm{O}) \mathrm{c} 1 \mathrm{c}(\mathrm{O}) \mathrm{c}(\mathrm{N}(=\mathrm{O})=\mathrm{O}) \mathrm{cc}(\mathrm{c} 1 \\
) \mathrm{C}(\mathrm{C})(\mathrm{C}) \mathrm{C}\end{array}$ & 1.80 & $1,2,14$ \\
\hline 165 & $\mathrm{p}$ & $87-86-5$ & pentachlorophenol & $\mathrm{Clclc}(\mathrm{Cl}) \mathrm{c}(\mathrm{Cl}) \mathrm{c}(\mathrm{c}(\mathrm{clCl}) \mathrm{Cl}) \mathrm{O}$ & 2.07 & $1,2,3,4,5,7,8,11,14$ \\
\hline 166 & $\mathrm{p}$ & $771-69-7$ & 1,2,3-trifluoro-4-nitrobenzene & $\mathrm{N}(=\mathrm{O})(=\mathrm{O}) \mathrm{c} 1 \mathrm{c}(\mathrm{F}) \mathrm{c}(\mathrm{F}) \mathrm{c}(\mathrm{cc} 1) \mathrm{F}$ & 1.89 & 1 \\
\hline 167 & $\mathrm{p}$ & $619-24-9$ & 3-nitrobenzonitrile & $\mathrm{N}(=\mathrm{O})(=\mathrm{O}) \mathrm{c} 1 \mathrm{cc}(\mathrm{CHN}) \mathrm{ccc} 1$ & 0.45 & $1,2,3$ \\
\hline 168 & $\mathrm{p}$ & $879-39-0$ & 2,3,4,5-tetrachloronitrobenzene & $\mathrm{N}(=\mathrm{O})(=\mathrm{O}) \mathrm{clc}(\mathrm{Cl}) \mathrm{c}(\mathrm{Cl}) \mathrm{c}(\mathrm{c}(\mathrm{c} 1) \mathrm{Cl}) \mathrm{Cl}$ & 1.78 & 1,3 \\
\hline 169 & $\mathrm{p}$ & $99-65-0$ & 1,3-dinitrobenzene & $\mathrm{N}(=\mathrm{O})(=\mathrm{O}) \operatorname{clcc}(\mathrm{N}(=\mathrm{O})=\mathrm{O}) \operatorname{ccc} 1$ & 0.76 & $1,2,3$ \\
\hline 170 & $\mathrm{p}$ & $117-18-0$ & 2,3,5,6-tetrachloronitrobenzene & $\mathrm{N}(=\mathrm{O})(=\mathrm{O}) \mathrm{clc}(\mathrm{Cl}) \mathrm{c}(\mathrm{Cl}) \mathrm{cc}(\mathrm{c} 1 \mathrm{Cl}) \mathrm{Cl}$ & 1.82 & 1,3 \\
\hline 171 & $\mathrm{p}$ & $528-29-0$ & 1,2-dinitrobenzene & $\mathrm{N}(=\mathrm{O})(=\mathrm{O}) \mathrm{c} 1 \mathrm{c}(\mathrm{N}(=\mathrm{O})=\mathrm{O}) \operatorname{cccc} 1$ & 1.25 & $1,2,3$ \\
\hline 172 & $\mathrm{p}$ & $327-92-4$ & 1,5-difluoro-2,4-dinitrobenzene & $\mathrm{N}(=\mathrm{O})(=\mathrm{O}) \mathrm{c} 1 \mathrm{cc}(\mathrm{N}(=\mathrm{O})=\mathrm{O}) \mathrm{c}(\mathrm{cc} 1 \mathrm{~F}) \mathrm{F}$ & 2.08 & 1 \\
\hline 173 & $\mathrm{p}$ & $121-14-2$ & 1-methyl-2,4-dinitrobenzene & $\mathrm{N}(=\mathrm{O})(=\mathrm{O}) \operatorname{clcc}(\mathrm{N}(=\mathrm{O})=\mathrm{O}) \operatorname{ccc} 1 \mathrm{C}$ & 0.87 & $1,2,14$ \\
\hline 174 & $\mathrm{p}$ & $314-41-0$ & 2,3,4,6-tetrafluoronitrobenzene & $\mathrm{N}(=\mathrm{O})(=\mathrm{O}) \mathrm{c} 1 \mathrm{c}(\mathrm{F}) \mathrm{c}(\mathrm{F}) \mathrm{c}(\mathrm{cc} 1 \mathrm{~F}) \mathrm{F}$ & 1.87 & 1 \\
\hline 175 & $\mathrm{p}$ & $89-61-2$ & 2,5-dichloro-1-nitrobenzene & $\mathrm{N}(=\mathrm{O})(=\mathrm{O}) \mathrm{clc}(\mathrm{Cl}) \operatorname{ccc}(\mathrm{c} 1) \mathrm{Cl}$ & 1.13 & $1,2,3$ \\
\hline 176 & $\mathrm{p}$ & $709-49-9$ & 1-iodo-2,4-dinitrobenzene & $\mathrm{N}(=\mathrm{O})(=\mathrm{O}) \operatorname{c1cc}(\mathrm{N}(=\mathrm{O})=\mathrm{O}) \operatorname{ccc} 1 \mathrm{I}$ & 2.12 & 1 \\
\hline 177 & $\mathrm{p}$ & $3209-22-1$ & 2,3-dichloro-1-nitrobenzene & $\mathrm{N}(=\mathrm{O})(=\mathrm{O}) \mathrm{clc}(\mathrm{Cl}) \mathrm{c}(\mathrm{Cl}) \mathrm{ccc} 1$ & 1.07 & 1,2 \\
\hline 178 & $\mathrm{p}$ & $70-34-8$ & 1-fluoro-2,4-dinitrobenzene & $\mathrm{N}(=\mathrm{O})(=\mathrm{O}) \operatorname{clcc}(\mathrm{N}(=\mathrm{O})=\mathrm{O}) \operatorname{ccc} 1 \mathrm{~F}$ & 1.71 & 1 \\
\hline 179 & $\mathrm{p}$ & $611-06-3$ & 2,4-dichloro-1-nitrobenzene & $\mathrm{N}(=\mathrm{O})(=\mathrm{O}) \mathrm{clc}(\mathrm{Cl}) \mathrm{cc}(\mathrm{cc} 1) \mathrm{Cl}$ & 0.99 & 1,2 \\
\hline 180 & $\mathrm{p}$ & $880-78-4$ & pentafluoronitrobenzene & $\mathrm{N}(=\mathrm{O})(=\mathrm{O}) \mathrm{c} 1 \mathrm{c}(\mathrm{F}) \mathrm{c}(\mathrm{F}) \mathrm{c}(\mathrm{c}(\mathrm{c} 1 \mathrm{~F}) \mathrm{F}) \mathrm{F}$ & 2.43 & 1 \\
\hline 181 & $\mathrm{p}$ & $618-62-2$ & 3,5-dichloro-1-nitrobenzene & $\mathrm{N}(=\mathrm{O})(=\mathrm{O}) \mathrm{c} 1 \mathrm{cc}(\mathrm{Cl}) \mathrm{cc}(\mathrm{c} 1) \mathrm{Cl}$ & 1.13 & 1 \\
\hline 182 & $\mathrm{p}$ & $584-48-5$ & 1-bromo-2,4-dinitrobenzene & $\mathrm{N}(=\mathrm{O})(=\mathrm{O}) \operatorname{c1} \operatorname{cc}(\mathrm{N}(=\mathrm{O})=\mathrm{O}) \operatorname{ccc} 1 \mathrm{Br}$ & 2.31 & 1 \\
\hline 183 & $\mathrm{p}$ & $99-54-7$ & 3,4-dichloro-1-nitrobenzene & $\mathrm{N}(=\mathrm{O})(=\mathrm{O}) \mathrm{c} 1 \mathrm{cc}(\mathrm{Cl}) \mathrm{c}(\mathrm{cc} 1) \mathrm{Cl}$ & 1.16 & 1 \\
\hline 184 & $\mathrm{p}$ & $6306-39-4$ & 1,2-dichloro-4,5-dinitrobenzene & $\mathrm{N}(=\mathrm{O})(=\mathrm{O}) \mathrm{clc}(\mathrm{N}(=\mathrm{O})=\mathrm{O}) \mathrm{cc}(\mathrm{c}(\mathrm{c} 1) \mathrm{C}$ & 2.21 & 1 \\
\hline
\end{tabular}




\begin{tabular}{|c|c|c|c|c|c|c|}
\hline & & & & 1) $\mathrm{Cl}$ & & \\
\hline 185 & $\mathrm{p}$ & $608-71-9$ & pentabromophenol & $\mathrm{Brc} 1 \mathrm{c}(\mathrm{Br}) \mathrm{c}(\mathrm{Br}) \mathrm{c}(\mathrm{c}(\mathrm{c} 1 \mathrm{Br}) \mathrm{Br}) \mathrm{O}$ & 2.66 & $1,2,520$ \\
\hline 186 & $\mathrm{p}$ & $28689-08-9$ & 1,5-dichloro-2,3-dinitrobenzene & $\begin{array}{l}\mathrm{N}(=\mathrm{O})(=\mathrm{O}) \mathrm{clc}(\mathrm{N}(=\mathrm{O})=\mathrm{O}) \mathrm{cc}(\mathrm{cc} 1 \mathrm{Cl}) \\
\mathrm{Cl}\end{array}$ & 2.42 & $1,2,14$ \\
\hline 187 & $\mathrm{p}$ & $97-02-9$ & 2,4-dinitroaniline & $\mathrm{N}(=\mathrm{O})(=\mathrm{O}) \operatorname{c} 1 \mathrm{cc}(\mathrm{N}(=\mathrm{O})=\mathrm{O}) \operatorname{ccc} 1 \mathrm{~N}$ & 0.72 & 1,2 \\
\hline 188 & $\mathrm{p}$ & $97-00-7$ & 1-chloro-2,4-dinitrobenzene & $\mathrm{N}(=\mathrm{O})(=\mathrm{O}) \operatorname{clcc}(\mathrm{N}(=\mathrm{O})=\mathrm{O}) \operatorname{ccc} 1 \mathrm{Cl}$ & 2.16 & 1,2 \\
\hline 189 & $\mathrm{p}$ & $606-22-4$ & 2,6-dinitroaniline & $\mathrm{N}(=\mathrm{O})(=\mathrm{O}) \mathrm{c} 1 \mathrm{c}(\mathrm{N}) \mathrm{c}(\mathrm{N}(=\mathrm{O})=\mathrm{O}) \mathrm{ccc} 1$ & 0.84 & 1 \\
\hline 190 & $\mathrm{p}$ & $6284-83-9$ & 1,3,5-trichloro-2,4-dinitrobenzene & $\begin{array}{l}\mathrm{Clc1c}(\mathrm{N}(=\mathrm{O})=\mathrm{O}) \mathrm{c}(\mathrm{Cl}) \operatorname{cc}(\mathrm{c} 1 \mathrm{~N}(=\mathrm{O})= \\
\mathrm{O}) \mathrm{Cl}\end{array}$ & 2.19 & 1 \\
\hline 191 & $\mathrm{p}$ & $350-30-1$ & 3-chloro-4-fluoro-1-nitrobenzene & $\mathrm{N}(=\mathrm{O})(=\mathrm{O}) \mathrm{c} 1 \mathrm{cc}(\mathrm{Cl}) \mathrm{c}(\mathrm{cc} 1) \mathrm{F}$ & 0.80 & 1 \\
\hline 192 & $\mathrm{p}$ & $2678-21-9$ & 1,3-dinitro-2,4,5-trichlorobenzene & $\begin{array}{l}\mathrm{N}(=\mathrm{O})(=\mathrm{O}) \mathrm{clc}(\mathrm{Cl}) \mathrm{c}(\mathrm{N}(=\mathrm{O})=\mathrm{O}) \mathrm{cc}(\mathrm{c} \\
1 \mathrm{Cl}) \mathrm{Cl}\end{array}$ & 2.60 & 1 \\
\hline 193 & $\mathrm{p}$ & $79544-31-3$ & 3,4-dinitrobenzyl alcohol & $\begin{array}{l}\mathrm{N}(=\mathrm{O})(=\mathrm{O}) \mathrm{c} 1 \mathrm{c}(\mathrm{N}(=\mathrm{O})=\mathrm{O}) \operatorname{ccc}(\mathrm{c} 1) \mathrm{C} \\
\mathrm{O}\end{array}$ & 1.09 & 1,3 \\
\hline 194 & $\mathrm{p}$ & $1930-72-9$ & 4-chloro-3,5-dinitrobenzonitrile & $\begin{array}{l}\mathrm{N}(=\mathrm{O})(=\mathrm{O}) \mathrm{clc}(\mathrm{Cl}) \mathrm{c}(\mathrm{N}(=\mathrm{O})=\mathrm{O}) \mathrm{cc}(\mathrm{c} \\
1) \mathrm{C \# N}\end{array}$ & 2.66 & 1 \\
\hline 195 & $\mathrm{p}$ & $100-25-4$ & 1,4-dinitrobenzene & $\mathrm{N}(=\mathrm{O})(=\mathrm{O}) \operatorname{clccc}(\operatorname{cc} 1) \mathrm{N}(=\mathrm{O})=\mathrm{O}$ & 1.30 & $1,2,3,14$ \\
\hline 196 & $\mathrm{p}$ & $20098-38-8$ & 1,4-dinitrotetrachlorobenzene & $\begin{array}{l}\mathrm{N}(=\mathrm{O})(=\mathrm{O}) \mathrm{clc}(\mathrm{Cl}) \mathrm{c}(\mathrm{Cl}) \mathrm{c}(\mathrm{c}(\mathrm{clCl}) \mathrm{Cl}) \\
\mathrm{N}(=\mathrm{O})=\mathrm{O}\end{array}$ & 2.82 & 1 \\
\hline 197 & $\mathrm{n}$ & $1877-77-6$ & 3-aminobenzyl alcohol & $\mathrm{C}(\mathrm{O}) \mathrm{c} 1 \mathrm{cc}(\mathrm{N}) \mathrm{ccc} 1$ & -1.28 & 2 \\
\hline 198 & $\mathrm{p}$ & $95-64-7$ & 3,4-dimethylaniline & $\operatorname{Cc} 1 \mathrm{c}(\mathrm{C}) \operatorname{ccc}(\mathrm{c} 1) \mathrm{N}$ & -0.08 & 2 \\
\hline 199 & $\mathrm{p}$ & $99-88-7$ & 4-isopropylaniline & $\mathrm{C}(\mathrm{C})(\mathrm{C}) \mathrm{c} 1 \mathrm{ccc}(\mathrm{cc} 1) \mathrm{N}$ & 0.23 & 2 \\
\hline 200 & $\mathrm{n}$ & $501-94-0$ & 4-hydroxyphenethyl alcohol & $\mathrm{C}(\mathrm{CO}) \mathrm{c} 1 \mathrm{ccc}(\mathrm{cc} 1) \mathrm{O}$ & -0.65 & $2,3,4,5,7,8,11$ \\
\hline 201 & $\mathrm{p}$ & $70-70-2$ & 4-hydroxypropiophenone & $\mathrm{C}(\mathrm{CC})(=\mathrm{O}) \mathrm{c} 1 \mathrm{ccc}(\mathrm{cc} 1) \mathrm{O}$ & 0.11 & $3,4,5,7,8,11$ \\
\hline 202 & $\mathrm{p}$ & $156-41-2$ & 2-(4-chlorophenyl)ethylamine & $\mathrm{C}(\mathrm{CN}) \mathrm{c} 1 \mathrm{ccc}(\mathrm{cc} 1) \mathrm{Cl}$ & 0.19 & 2 \\
\hline 203 & $\mathrm{p}$ & $121-33-5$ & 3-Methoxy-4-hydroxybenzaldehyde & $\mathrm{O}(\mathrm{C}) \mathrm{c} 1 \mathrm{cc}(\mathrm{C}=\mathrm{O}) \operatorname{ccc} 1 \mathrm{O}$ & -0.11 & $3,4,8,10$ \\
\hline 204 & $\mathrm{p}$ & $540-38-5$ & 4-iodophenol & $\mathrm{Ic} 1 \mathrm{ccc}(\mathrm{cc} 1) \mathrm{O}$ & 0.84 & $2,3,4,5,7,8$ \\
\hline 205 & $\mathrm{p}$ & $90-90-4$ & 4-bromobenzophenone & $\mathrm{C}(=\mathrm{O})(\mathrm{c} 1 \mathrm{ccc}(\mathrm{cc} 1) \mathrm{Br}) \mathrm{c} 1 \mathrm{ccccc} 1$ & 1.34 & 2 \\
\hline 206 & $\mathrm{p}$ & $89-62-3$ & 4-methyl-2-nitroaniline & $\mathrm{N}(=\mathrm{O})(=\mathrm{O}) \mathrm{c} 1 \mathrm{c}(\mathrm{N}) \operatorname{ccc}(\mathrm{c} 1) \mathrm{C}$ & 0.40 & 2 \\
\hline 207 & $\mathrm{p}$ & $7530-27-0$ & 4-bromo-6-chloro-2-methylphenol & $\mathrm{Oc} 1 \mathrm{c}(\mathrm{C}) \mathrm{cc}(\mathrm{cc} 1 \mathrm{Cl}) \mathrm{Br}$ & 1.29 & $3,4,5,7,8,11$ \\
\hline 208 & $\mathrm{p}$ & $615-58-7$ & 2,4-dibromophenol & $\mathrm{Brc} 1 \mathrm{c}(\mathrm{O}) \mathrm{ccc}(\mathrm{c} 1) \mathrm{Br}$ & 1.21 & $2,3,4,5,7,8,11$ \\
\hline 209 & $\mathrm{p}$ & $697-86-9$ & 2,4-Dichloro-6-bromoaniline & $\mathrm{Nc} 1 \mathrm{c}(\mathrm{Br}) \mathrm{cc}(\mathrm{cc} 1 \mathrm{Cl}) \mathrm{Cl}$ & 1.44 & 2 \\
\hline 210 & $\mathrm{p}$ & $771-61-9$ & pentafluorophenol & $\mathrm{Fclc}(\mathrm{F}) \mathrm{c}(\mathrm{F}) \mathrm{c}(\mathrm{c}(\mathrm{c} 1 \mathrm{~F}) \mathrm{F}) \mathrm{O}$ & 1.40 & $2,3,4,5,7,8,11,14$ \\
\hline 211 & $\mathrm{p}$ & $100-14-1$ & 4-nitrobenzyl chloride & $\mathrm{N}(=\mathrm{O})(=\mathrm{O}) \mathrm{c} 1 \mathrm{ccc}(\mathrm{cc} 1) \mathrm{CCl}$ & 1.10 & 2,3 \\
\hline 212 & $\mathrm{p}$ & $7147-89-9$ & 4-chloro-6-nitro-3-methylphenol & $\mathrm{N}(=\mathrm{O})(=\mathrm{O}) \mathrm{c} 1 \mathrm{c}(\mathrm{O}) \mathrm{cc}(\mathrm{c}(\mathrm{c} 1) \mathrm{Cl}) \mathrm{C}$ & 1.34 & $3,4,5,7,8,11$ \\
\hline 213 & $\mathrm{p}$ & $305-85-1$ & 2,6-diiodo-4-nitrophenol & $\mathrm{N}(=\mathrm{O})(=\mathrm{O}) \mathrm{c} 1 \mathrm{cc}(\mathrm{I}) \mathrm{c}(\mathrm{c}(\mathrm{c} 1) \mathrm{I}) \mathrm{O}$ & 1.84 & $2,3,4,5,7,8,11$ \\
\hline 214 & $\mathrm{p}$ & $609-89-2$ & 2,4-dichloro-6-nitrophenol & $\mathrm{N}(=\mathrm{O})(=\mathrm{O}) \mathrm{clc}(\mathrm{O}) \mathrm{c}(\mathrm{Cl}) \mathrm{cc}(\mathrm{c} 1) \mathrm{Cl}$ & 1.71 & $2,4,5,7,8,11$ \\
\hline 215 & $\mathrm{n}$ & $552-16-9$ & 2-nitrobenzoic acid & $\mathrm{C}(=\mathrm{O})(\mathrm{O}) \mathrm{c} 1 \mathrm{c}(\mathrm{N}(=\mathrm{O})=\mathrm{O}) \mathrm{cccc} 1$ & -1.64 & 2,3 \\
\hline 216 & $\mathrm{n}$ & $121-92-6$ & 3-nitrobenzoic acid & $\mathrm{C}(=\mathrm{O})(\mathrm{O}) \mathrm{c} 1 \mathrm{cc}(\mathrm{N}(=\mathrm{O})=\mathrm{O}) \operatorname{ccc} 1$ & -1.09 & 3 \\
\hline 217 & $\mathrm{n}$ & $620-24-6$ & 3-hydroxybenzyl alcohol & $\mathrm{C}(\mathrm{O}) \mathrm{c} 1 \mathrm{cc}(\mathrm{O}) \mathrm{ccc} 1$ & -1.04 & $2,3,4,5,7,8,11$ \\
\hline
\end{tabular}




\begin{tabular}{|c|c|c|c|c|c|c|}
\hline 218 & $\mathrm{n}$ & $99-96-7$ & 4-hydroxybenzoic acid & $\mathrm{C}(=\mathrm{O})(\mathrm{O}) \mathrm{c} 1 \mathrm{ccc}(\mathrm{cc} 1) \mathrm{O}$ & -1.02 & 4,8 \\
\hline 219 & $\mathrm{n}$ & $90-01-7$ & 2-hydroxybenzyl alcohol & $\mathrm{C}(\mathrm{O}) \mathrm{c} 1 \mathrm{c}(\mathrm{O}) \mathrm{cccc} 1$ & -0.95 & 4 \\
\hline 220 & $\mathrm{n}$ & $62-23-7$ & 4-nitrobenzoic acid & $\mathrm{C}(=\mathrm{O})(\mathrm{O}) \mathrm{c} 1 \mathrm{ccc}(\mathrm{cc} 1) \mathrm{N}(=\mathrm{O})=\mathrm{O}$ & -0.86 & 3 \\
\hline 221 & $\mathrm{n}$ & $100-48-1$ & 4-cyanopyridine & $\mathrm{C}(\# \mathrm{~N}) \mathrm{c} 1 \mathrm{ccncc} 1$ & -0.82 & 3 \\
\hline 222 & $\mathrm{n}$ & $103-90-2$ & 4-hydroxyacetanilide & $\mathrm{N}(\mathrm{C}(\mathrm{C})=\mathrm{O}) \mathrm{c} 1 \operatorname{ccc}(\mathrm{cc} 1) \mathrm{O}$ & -0.82 & $4,5,7,8,11$ \\
\hline 223 & $\mathrm{n}$ & $99-06-9$ & 3-hydroxybenzoic acid & $\mathrm{C}(=\mathrm{O})(\mathrm{O}) \mathrm{c} 1 \mathrm{cc}(\mathrm{O}) \mathrm{ccc} 1$ & -0.81 & $2,3,4,5,8,11$ \\
\hline 224 & $\mathrm{n}$ & $100-70-9$ & 2-cyanopyridine & $\mathrm{C}(\# \mathrm{~N}) \mathrm{c} 1 \mathrm{cccc} 1$ & -0.79 & 3 \\
\hline 225 & $\mathrm{n}$ & $619-57-8$ & 4-hydroxybenzamide & $\mathrm{C}(\mathrm{N})(=\mathrm{O}) \mathrm{c} 1 \mathrm{ccc}(\mathrm{cc} 1) \mathrm{O}$ & -0.78 & $3,4,5,7,8$ \\
\hline 226 & $\mathrm{n}$ & $100-54-9$ & 3-cyanopyridine & $\mathrm{C}(\# \mathrm{~N}) \mathrm{c} 1 \mathrm{cccnc} 1$ & -0.74 & 3 \\
\hline 227 & $\mathrm{n}$ & $610-15-1$ & 2-nitrobenzamide & $\mathrm{C}(\mathrm{N})(=\mathrm{O}) \mathrm{c} 1 \mathrm{c}(\mathrm{N}(=\mathrm{O})=\mathrm{O}) \operatorname{ccc} 1$ & -0.72 & 2,3 \\
\hline 228 & $\mathrm{n}$ & $769-28-8$ & $\begin{array}{l}\text { 3-cyano-4,6-dimethyl-2-hydroxypyridi } \\
\text { ne }\end{array}$ & $\mathrm{C}(\# \mathrm{~N}) \mathrm{clc}(\mathrm{C}) \mathrm{cc}([\mathrm{nH}] \mathrm{c} 1=\mathrm{O}) \mathrm{C}$ & -0.70 & 3 \\
\hline 229 & $\mathrm{n}$ & $91-10-1$ & 2,6-dimethoxyphenol & $\mathrm{O}(\mathrm{C}) \mathrm{c} 1 \mathrm{c}(\mathrm{O}) \mathrm{c}(\mathrm{OC}) \mathrm{ccc} 1$ & -0.60 & $2,3,4,5,7,8,11$ \\
\hline 230 & $\mathrm{n}$ & $591-27-5$ & 3-aminophenol & $\mathrm{Nc1cc}(\mathrm{O}) \mathrm{ccc} 1$ & -0.52 & $2,3,4,5,7,8,11$ \\
\hline 231 & $\mathrm{n}$ & $100-47-0$ & benzonitrile & $\mathrm{C}(\# \mathrm{~N}) \mathrm{c} 1 \mathrm{ccccc} 1$ & -0.52 & 2,3 \\
\hline 232 & $\mathrm{n}$ & $69-72-7$ & salicylic acid & $\mathrm{C}(=\mathrm{O})(\mathrm{O}) \mathrm{c} 1 \mathrm{c}(\mathrm{O}) \mathrm{cccc} 1$ & -0.51 & $2,3,4,5,7,8,11$ \\
\hline 233 & $\mathrm{n}$ & $90-05-1$ & 2-methoxyphenol & $\mathrm{O}(\mathrm{C}) \mathrm{c} 1 \mathrm{c}(\mathrm{O}) \mathrm{cccc} 1$ & -0.51 & $3,4,5,7,8,11$ \\
\hline 234 & $\mathrm{n}$ & $1885-29-6$ & 2-aminobenzonitrile & $\mathrm{C}(\# \mathrm{~N}) \mathrm{c} 1 \mathrm{c}(\mathrm{N}) \mathrm{cccc} 1$ & -0.50 & 2,3 \\
\hline 235 & $\mathrm{p}$ & $2237-30-1$ & 3-aminobenzonitrile & $\mathrm{C}(\# \mathrm{~N}) \mathrm{c} 1 \mathrm{cc}(\mathrm{N}) \mathrm{ccc} 1$ & -0.47 & 2,3 \\
\hline 236 & $\mathrm{p}$ & $121-71-1$ & 3-hydroxyacetophenone & $\mathrm{C}(\mathrm{C})(=\mathrm{O}) \mathrm{c} 1 \mathrm{cc}(\mathrm{O}) \mathrm{ccc} 1$ & -0.38 & 2,4 \\
\hline 237 & $\mathrm{p}$ & $3034-34-2$ & 4-cyanobenzamide & $\mathrm{C}(\mathrm{N})(=\mathrm{O}) \mathrm{c} 1 \mathrm{ccc}(\mathrm{cc} 1) \mathrm{C \# N}$ & -0.38 & 2,3 \\
\hline 238 & $\mathrm{p}$ & $1443-80-7$ & 4-cyanoacetophenone & $\mathrm{C}(\mathrm{C})(=\mathrm{O}) \mathrm{c} 1 \mathrm{ccc}(\mathrm{cc} 1) \mathrm{C} \# \mathrm{~N}$ & -0.37 & 2 \\
\hline 239 & $\mathrm{p}$ & $94-71-3$ & 2-ethoxyphenol & $\mathrm{O}(\mathrm{CC}) \mathrm{c} 1 \mathrm{c}(\mathrm{O}) \mathrm{cccc} 1$ & -0.36 & $2,3,4,5,7,8,11$ \\
\hline 240 & $\mathrm{p}$ & $91-15-6$ & 1,2-dicyanobenzene & $\mathrm{C}(\# \mathrm{~N}) \mathrm{c} 1 \mathrm{c}(\mathrm{CHN}) \mathrm{cccc} 1$ & -0.34 & 2 \\
\hline 241 & $\mathrm{p}$ & $99-93-4$ & 4-hydroxyacetophenone & $\mathrm{C}(\mathrm{C})(=\mathrm{O}) \mathrm{c} 1 \mathrm{ccc}(\mathrm{cc} 1) \mathrm{O}$ & -0.30 & $2,4,8$ \\
\hline 242 & $\mathrm{p}$ & $1194-02-1$ & 4-fluorobenzonitrile & $\mathrm{C}(\# \mathrm{~N}) \mathrm{c} 1 \mathrm{ccc}(\mathrm{cc} 1) \mathrm{F}$ & -0.26 & 2,3 \\
\hline 243 & $\mathrm{p}$ & $620-22-4$ & 3-tolunitrile & $\mathrm{C}(\# \mathrm{~N}) \mathrm{c} 1 \mathrm{cc}(\mathrm{C}) \mathrm{ccc} 1$ & -0.25 & 2 \\
\hline 244 & $\mathrm{p}$ & $65-45-2$ & salicylamide & $\mathrm{C}(\mathrm{N})(=\mathrm{O}) \mathrm{c} 1 \mathrm{c}(\mathrm{O}) \mathrm{cccc} 1$ & -0.24 & $2,4,8$ \\
\hline 245 & $\mathrm{p}$ & $619-25-0$ & 3-nitrobenzyl alcohol & $\mathrm{N}(=\mathrm{O})(=\mathrm{O}) \mathrm{c} 1 \mathrm{cc}(\mathrm{CO}) \mathrm{ccc} 1$ & -0.22 & 2,3 \\
\hline 246 & $\mathrm{p}$ & $645-09-0$ & 3-nitrobenzamide & $\mathrm{C}(\mathrm{N})(=\mathrm{O}) \mathrm{c} 1 \mathrm{cc}(\mathrm{N}(=\mathrm{O})=\mathrm{O}) \operatorname{ccc} 1$ & -0.19 & 2,3 \\
\hline 247 & $\mathrm{p}$ & $621-42-1$ & 3-acetamidophenol & $\mathrm{N}(\mathrm{C}(\mathrm{C})=\mathrm{O}) \mathrm{c} 1 \mathrm{cc}(\mathrm{O}) \mathrm{ccc} 1$ & -0.16 & $3,4,5,7,8,11$ \\
\hline 248 & $\mathrm{p}$ & $29663-11-4$ & 2,4,6-trinitrophenol & $\begin{array}{l}\mathrm{N}(=\mathrm{O})(=\mathrm{O}) \mathrm{c} 1 \mathrm{c}(\mathrm{O}) \mathrm{c}(\mathrm{N}(=\mathrm{O})=\mathrm{O}) \mathrm{cc}(\mathrm{c} 1 \\
\mathrm{N}(=\mathrm{O})=\mathrm{O}\end{array}$ & -0.16 & $3,4,5,7,8,11$ \\
\hline 249 & $\mathrm{p}$ & $612-25-9$ & 2-nitrobenzyl alcohol & $\mathrm{C}(\mathrm{O}) \mathrm{c} 1 \mathrm{c}(\mathrm{N}(=\mathrm{O})=\mathrm{O}) \operatorname{cccc} 1$ & -0.16 & 2,3 \\
\hline 250 & $\mathrm{p}$ & $621-59-0$ & Isovanillin & $\mathrm{C}(=\mathrm{O}) \mathrm{c} 1 \mathrm{cc}(\mathrm{O}) \mathrm{c}(\mathrm{cc} 1) \mathrm{OC}$ & -0.14 & $4,8,10$ \\
\hline 251 & $\mathrm{p}$ & $104-85-8$ & 4-tolunitrile & $\mathrm{C}(\# \mathrm{~N}) \mathrm{c} 1 \mathrm{ccc}(\mathrm{cc} 1) \mathrm{C}$ & -0.10 & 2,3 \\
\hline 252 & $\mathrm{p}$ & $500-99-2$ & 3,5-dimethoxyphenol & $\mathrm{O}(\mathrm{C}) \mathrm{c} 1 \mathrm{cc}(\mathrm{OC}) \mathrm{cc}(\mathrm{c} 1) \mathrm{O}$ & -0.09 & $2,3,4,5,7,8,11$ \\
\hline 253 & $\mathrm{p}$ & $123-30-8$ & 4-aminophenol & $\mathrm{Nc} 1 \mathrm{ccc}(\mathrm{cc} 1) \mathrm{O}$ & -0.08 & $2,3,4,5,7,8,11$ \\
\hline 254 & $\mathrm{p}$ & $873-62-1$ & 3-cyanophenol & $\mathrm{C}(\# \mathrm{~N}) \mathrm{c} 1 \mathrm{cc}(\mathrm{O}) \mathrm{ccc} 1$ & -0.06 & $2,3,4,5,7,8,11$ \\
\hline
\end{tabular}




\begin{tabular}{|c|c|c|c|c|c|c|}
\hline 255 & $\mathrm{p}$ & $766-84-7$ & 3-chlorobenzonitrile & $\mathrm{C}(\# \mathrm{~N}) \mathrm{c} 1 \mathrm{cc}(\mathrm{Cl}) \mathrm{ccc} 1$ & -0.06 & 2,3 \\
\hline 256 & $\mathrm{p}$ & $1129-35-7$ & methyl-4-cyanobenzoate & $\mathrm{C}(\mathrm{OC})(=\mathrm{O}) \mathrm{c} 1 \mathrm{ccc}(\mathrm{cc} 1) \mathrm{C} \# \mathrm{~N}$ & -0.06 & 2 \\
\hline 257 & $\mathrm{p}$ & $19438-10-9$ & methyl-3-hydroxybenzoate & $\mathrm{C}(\mathrm{OC})(=\mathrm{O}) \mathrm{c} 1 \mathrm{cc}(\mathrm{O}) \mathrm{ccc} 1$ & -0.05 & $2,3,4,5,7,8,11$ \\
\hline 258 & $\mathrm{p}$ & $24964-64-5$ & 3-cyanobenzaldehyde & $\mathrm{C}(\# \mathrm{~N}) \mathrm{c} 1 \mathrm{cc}(\mathrm{C}=\mathrm{O}) \mathrm{ccc} 1$ & -0.02 & $2,3,10$ \\
\hline 259 & $\mathrm{p}$ & $623-03-0$ & 4-chlorobenzonitrile & $\mathrm{C}(\# \mathrm{~N}) \mathrm{c} 1 \mathrm{ccc}(\mathrm{cc} 1) \mathrm{Cl}$ & 0.00 & 3 \\
\hline 260 & $\mathrm{p}$ & $622-62-8$ & 4-ethoxyphenol & $\mathrm{O}(\mathrm{CC}) \mathrm{c} 1 \mathrm{ccc}(\mathrm{cc} 1) \mathrm{O}$ & 0.01 & $2,3,4,5,7,8,11$ \\
\hline 261 & $\mathrm{p}$ & $371-41-5$ & 4-fluorophenol & $\mathrm{Fclccc}(\mathrm{cc} 1) \mathrm{O}$ & 0.02 & $2,3,4,5,7,8,11$ \\
\hline 262 & $\mathrm{p}$ & $611-20-1$ & 2-cyanophenol & $\mathrm{C}(\# \mathrm{~N}) \mathrm{c} 1 \mathrm{c}(\mathrm{O}) \operatorname{cccc} 1$ & 0.03 & $2,3,4,5,8,11$ \\
\hline 263 & $\mathrm{p}$ & $105-07-7$ & 4-cyanobenzaldehyde & $\mathrm{C}(\# \mathrm{~N}) \mathrm{c} 1 \operatorname{ccc}(\operatorname{cc} 1) \mathrm{C}=\mathrm{O}$ & 0.04 & $2,3,10$ \\
\hline 264 & $\mathrm{p}$ & $1527-89-5$ & 3-methoxybenzonitrile & $\mathrm{C}(\# \mathrm{~N}) \mathrm{c} 1 \mathrm{cc}(\mathrm{OC}) \mathrm{ccc} 1$ & 0.05 & 2,3 \\
\hline 265 & $\mathrm{p}$ & $95-87-4$ & 2,5-dimethylphenol & $\mathrm{Oc} 1 \mathrm{c}(\mathrm{C}) \operatorname{ccc}(\mathrm{c} 1) \mathrm{C}$ & 0.08 & $3,4,5,7,8,11$ \\
\hline 266 & $\mathrm{p}$ & $582-24-1$ & 2-hydroxyacetophenone & $\mathrm{C}(\mathrm{CO})(=\mathrm{O}) \mathrm{c} 1 \mathrm{ccccc} 1$ & 0.08 & $3,4,5,7,8,11$ \\
\hline 267 & $\mathrm{p}$ & $99-76-3$ & methyl-4-hydroxybenzoate & $\mathrm{C}(\mathrm{OC})(=\mathrm{O}) \mathrm{c} 1 \mathrm{ccc}(\mathrm{cc} 1) \mathrm{O}$ & 0.08 & $2,3,4,5,7,8,11$ \\
\hline 268 & $\mathrm{p}$ & $100-83-4$ & 3-hydroxybenzaldehyde & $\mathrm{C}(=\mathrm{O}) \mathrm{c} 1 \mathrm{cc}(\mathrm{O}) \mathrm{ccc} 1$ & 0.09 & $2,3,4,5,7,8,10,11$ \\
\hline 269 & $\mathrm{p}$ & $874-90-8$ & 4-methoxybenzonitrile & $\mathrm{C}(\# \mathrm{~N}) \mathrm{c} 1 \mathrm{ccc}(\mathrm{cc} 1) \mathrm{OC}$ & 0.10 & 2,3 \\
\hline 270 & $\mathrm{p}$ & $619-73-8$ & 4-nitrobenzyl alcohol & $\mathrm{N}(=\mathrm{O})(=\mathrm{O}) \mathrm{c} 1 \mathrm{ccc}(\mathrm{cc} 1) \mathrm{CO}$ & 0.10 & 2,3 \\
\hline 271 & $\mathrm{p}$ & $108-68-9$ & 3,5-dimethylphenol & $\mathrm{Cc} 1 \mathrm{cc}(\mathrm{C}) \mathrm{cc}(\mathrm{c} 1) \mathrm{O}$ & 0.11 & $2,3,4,5,7,8,11$ \\
\hline 272 & $\mathrm{p}$ & $95-65-8$ & 3,4-dimethylphenol & $\mathrm{Cc} 1 \mathrm{c}(\mathrm{C}) \operatorname{ccc}(\mathrm{c} 1) \mathrm{O}$ & 0.12 & $2,3,4,5,7,8,11$ \\
\hline 273 & $\mathrm{p}$ & $526-75-0$ & 2,3-dimethylphenol & $\mathrm{Cc} 1 \mathrm{c}(\mathrm{C}) \operatorname{cccc} 1 \mathrm{O}$ & 0.12 & $2,3,4,5,8,11$ \\
\hline 274 & $\mathrm{p}$ & $95-86-3$ & 2,4-diaminophenol & $\operatorname{Nclc}(\mathrm{O}) \operatorname{ccc}(\mathrm{c} 1) \mathrm{N}$ & 0.13 & $3,4,5,7,8,11$ \\
\hline 275 & $\mathrm{p}$ & $555-21-5$ & 4-nitrophenylacetonitrile & $\mathrm{N}(=\mathrm{O})(=\mathrm{O}) \mathrm{c} 1 \operatorname{ccc}(\operatorname{cc} 1) \mathrm{CC} \# \mathrm{~N}$ & 0.13 & 2,3 \\
\hline 276 & $\mathrm{p}$ & $99-61-6$ & 3-nitrobenzaldehyde & $\mathrm{N}(=\mathrm{O})(=\mathrm{O}) \mathrm{c} 1 \mathrm{cc}(\mathrm{C}=\mathrm{O}) \operatorname{ccc} 1$ & 0.14 & $2,3,10$ \\
\hline 277 & $\mathrm{p}$ & $134-96-3$ & syringaldehyde & $\mathrm{O}(\mathrm{C}) \mathrm{c} 1 \mathrm{c}(\mathrm{O}) \mathrm{c}(\mathrm{OC}) \mathrm{cc}(\mathrm{c} 1) \mathrm{C}=\mathrm{O}$ & 0.17 & $3,4,5,7,8,11$ \\
\hline 278 & $\mathrm{p}$ & $552-89-6$ & 2-nitrobenzaldehyde & $\mathrm{C}(=\mathrm{O}) \mathrm{c} 1 \mathrm{c}(\mathrm{N}(=\mathrm{O})=\mathrm{O}) \mathrm{cccc} 1$ & 0.17 & 3,10 \\
\hline 279 & $\mathrm{p}$ & $936-02-7$ & salicylhydrazide & $\mathrm{C}(\mathrm{NN})(=\mathrm{O}) \mathrm{c} 1 \mathrm{c}(\mathrm{O}) \mathrm{cccc} 1$ & 0.18 & $3,4,5,7,8,11$ \\
\hline 280 & $\mathrm{p}$ & $95-57-8$ & 2-chlorophenol & $\mathrm{Clc1c}(\mathrm{O}) \mathrm{cccc} 1$ & 0.18 & $2,3,4,5,7,8,11,14$ \\
\hline 281 & $\mathrm{p}$ & $619-80-7$ & 4-nitrobenzamide & $\mathrm{C}(\mathrm{N})(=\mathrm{O}) \mathrm{c} 1 \operatorname{ccc}(\operatorname{cc} 1) \mathrm{N}(=\mathrm{O})=\mathrm{O}$ & 0.18 & 3 \\
\hline 282 & $\mathrm{p}$ & $367-12-4$ & 2-fluorophenol & $\mathrm{Fclc}(\mathrm{O}) \mathrm{cccc} 1$ & 0.19 & $2,3,4,5,7,8,11$ \\
\hline 283 & $\mathrm{p}$ & $555-16-8$ & 4-nitrobenzaldehyde & $\mathrm{N}(=\mathrm{O})(=\mathrm{O}) \mathrm{c} 1 \operatorname{ccc}(\operatorname{cc} 1) \mathrm{C}=\mathrm{O}$ & 0.20 & $2,3,10$ \\
\hline 284 & $\mathrm{p}$ & $94-67-7$ & salicylaldoxime & $\mathrm{C}(=\mathrm{N} \backslash \mathrm{O}) \backslash \mathrm{c} 1 \mathrm{c}(\mathrm{O}) \operatorname{cccc} 1$ & 0.25 & $2,3,4,5,7,8,11$ \\
\hline 285 & $\mathrm{p}$ & $873-74-5$ & 4-aminobenzonitrile & $\mathrm{C}(\# \mathrm{~N}) \mathrm{c} 1 \mathrm{ccc}(\mathrm{cc} 1) \mathrm{N}$ & 0.25 & 2 \\
\hline 286 & $\mathrm{p}$ & $123-08-0$ & 4-hydroxybenzaldehyde & $\mathrm{C}(=\mathrm{O}) \mathrm{c} 1 \mathrm{ccc}(\mathrm{cc} 1) \mathrm{O}$ & 0.27 & $2,3,4,5,7,8,10,11$ \\
\hline 287 & $\mathrm{p}$ & $577-71-9$ & 3,4-dinitrophenol & $\mathrm{N}(=\mathrm{O})(=\mathrm{O}) \mathrm{c} 1 \mathrm{c}(\mathrm{N}(=\mathrm{O})=\mathrm{O}) \operatorname{cc}(\mathrm{cc} 1) \mathrm{O}$ & 0.27 & $3,4,5,7,8,11$ \\
\hline 288 & $\mathrm{p}$ & $873-32-5$ & 2-chlorobenzonitrile & $\mathrm{C}(\# \mathrm{~N}) \mathrm{c} 1 \mathrm{c}(\mathrm{Cl}) \operatorname{cccc} 1$ & 0.28 & 2,3 \\
\hline 289 & $\mathrm{p}$ & $623-00-7$ & 4-bromobenzonitrile & $\mathrm{C}(\# \mathrm{~N}) \mathrm{c} 1 \mathrm{ccc}(\mathrm{cc} 1) \mathrm{Br}$ & 0.29 & 3 \\
\hline 290 & $\mathrm{p}$ & $81-20-9$ & 1,3-dimethyl-2-nitrobenzene & $\mathrm{N}(=\mathrm{O})(=\mathrm{O}) \mathrm{c} 1 \mathrm{c}(\mathrm{C}) \operatorname{cccc} 1 \mathrm{C}$ & 0.30 & 3 \\
\hline 291 & $\mathrm{p}$ & $121-89-1$ & 3-nitroacetophenone & $\mathrm{C}(\mathrm{C})(=\mathrm{O}) \mathrm{c} 1 \mathrm{cc}(\mathrm{N}(=\mathrm{O})=\mathrm{O}) \operatorname{ccc} 1$ & 0.32 & 2 \\
\hline 292 & $\mathrm{p}$ & $95-56-7$ & 2-bromophenol & $\mathrm{Brc1c}(\mathrm{O}) \mathrm{cccc} 1$ & 0.33 & $3,4,5,7,8,11$ \\
\hline 293 & $\mathrm{p}$ & $42454-06-8$ & 5-hydroxy-2-nitrobenzaldehyde & $\mathrm{C}(=\mathrm{O}) \mathrm{c} 1 \mathrm{c}(\mathrm{N}(=\mathrm{O})=\mathrm{O}) \operatorname{ccc}(\mathrm{c} 1) \mathrm{O}$ & 0.33 & $1,3,4,5,7,8,11,13$ \\
\hline
\end{tabular}




\begin{tabular}{|c|c|c|c|c|c|c|}
\hline 294 & $\mathrm{p}$ & $2316-64-5$ & 5-bromo-2-hydroxybenzyl alcohol & $\mathrm{C}(\mathrm{O}) \mathrm{c} 1 \mathrm{c}(\mathrm{O}) \operatorname{ccc}(\mathrm{c} 1) \mathrm{Br}$ & 0.34 & $3,4,5,7,8,11$ \\
\hline 295 & $\mathrm{p}$ & $1199-46-8$ & 2-amino-4-tert-butylphenol & $\mathrm{C}(\mathrm{C})(\mathrm{C})(\mathrm{C}) \mathrm{c} 1 \mathrm{cc}(\mathrm{N}) \mathrm{c}(\mathrm{cc} 1) \mathrm{O}$ & 0.37 & $3,4,8$ \\
\hline 296 & $\mathrm{p}$ & $7153-22-2$ & ethyl-4-cyanobenzoate & $\mathrm{C}(\mathrm{OCC})(=\mathrm{O}) \mathrm{c} 1 \operatorname{ccc}(\operatorname{cc} 1) \mathrm{C} \# \mathrm{~N}$ & 0.37 & 2 \\
\hline 297 & $\mathrm{p}$ & $148-53-8$ & 3-methoxysalicylaldehyde & $\mathrm{C}(=\mathrm{O}) \mathrm{c} 1 \mathrm{c}(\mathrm{O}) \mathrm{c}(\mathrm{OC}) \mathrm{ccc} 1$ & 0.38 & $3,4,8,10$ \\
\hline 298 & $\mathrm{p}$ & $89-73-6$ & salicylhydroxamic acid & $\mathrm{C}(\mathrm{NO})(=\mathrm{O}) \mathrm{clc}(\mathrm{O}) \mathrm{cccc} 1$ & 0.38 & $3,4,5,7,8,11$ \\
\hline 299 & $\mathrm{p}$ & $372-20-3$ & 3-fluorophenol & Fc1cc(O)ccc1 & 0.38 & $3,4,5,7,8,11$ \\
\hline 300 & $\mathrm{p}$ & $586-78-7$ & 4-bromonitrobenzene & $\mathrm{N}(=\mathrm{O})(=\mathrm{O}) \mathrm{c} 1 \mathrm{ccc}(\mathrm{cc} 1) \mathrm{Br}$ & 0.38 & 2 \\
\hline 301 & $\mathrm{p}$ & $615-74-7$ & 2-chloro-5-methylphenol & $\mathrm{Oc} 1 \mathrm{c}(\mathrm{Cl}) \operatorname{ccc}(\mathrm{c} 1) \mathrm{C}$ & 0.39 & $2,3,4,5,7,8,11$ \\
\hline 302 & $\mathrm{p}$ & $619-50-1$ & methyl-4-nitrobenzoate & $\mathrm{C}(\mathrm{OC})(=\mathrm{O}) \operatorname{clccc}(\operatorname{cc} 1) \mathrm{N}(=\mathrm{O})=\mathrm{O}$ & 0.40 & 2 \\
\hline 303 & $\mathrm{p}$ & $1687-53-2$ & 5-amino-2-methoxyphenol & $\mathrm{O}(\mathrm{C}) \mathrm{c} 1 \mathrm{c}(\mathrm{O}) \mathrm{cc}(\mathrm{cc} 1) \mathrm{N}$ & 0.45 & $3,4,5,7,8,11$ \\
\hline 304 & $\mathrm{p}$ & $66-56-8$ & 2,3-dinitrophenol & $\mathrm{N}(=\mathrm{O})(=\mathrm{O}) \mathrm{c} 1 \mathrm{c}(\mathrm{N}(=\mathrm{O})=\mathrm{O}) \operatorname{ccc} 1 \mathrm{O}$ & 0.46 & $3,4,5,7,8,11$ \\
\hline 305 & $\mathrm{p}$ & $28177-48-2$ & 2,6-difluorophenol & Oc1c(F)ccc1F & 0.47 & $3,4,5,7,8,11$ \\
\hline 306 & $\mathrm{p}$ & $99-89-8$ & 4-isopropylphenol & $\mathrm{C}(\mathrm{C})(\mathrm{C}) \mathrm{c} 1 \mathrm{ccc}(\mathrm{cc} 1) \mathrm{O}$ & 0.47 & $2,3,4,5,7,8,11$ \\
\hline 307 & $\mathrm{p}$ & $7781-98-8$ & ethyl-3-hydroxybenzoate & $\mathrm{C}(\mathrm{OCC})(=\mathrm{O}) \mathrm{c} 1 \mathrm{cc}(\mathrm{O}) \mathrm{ccc} 1$ & 0.48 & $3,4,5,7,8,11$ \\
\hline 308 & $\mathrm{p}$ & $99-57-0$ & 2-amino-4-nitrophenol & $\mathrm{N}(=\mathrm{O})(=\mathrm{O}) \mathrm{c} 1 \mathrm{cc}(\mathrm{N}) \mathrm{c}(\mathrm{cc} 1) \mathrm{O}$ & 0.48 & $3,4,5,7,8,11$ \\
\hline 309 & $\mathrm{p}$ & $554-84-7$ & 3-nitrophenol & $\mathrm{N}(=\mathrm{O})(=\mathrm{O}) \mathrm{c} 1 \mathrm{cc}(\mathrm{O}) \mathrm{ccc} 1$ & 0.51 & $2,3,4,5,7,8,11$ \\
\hline 310 & $\mathrm{p}$ & $767-00-0$ & 4-cyanophenol & $\mathrm{C}(\# \mathrm{~N}) \mathrm{c} 1 \mathrm{ccc}(\mathrm{cc} 1) \mathrm{O}$ & 0.52 & $2,3,4,5,7,8,11$ \\
\hline 311 & $\mathrm{p}$ & $71022-43-0$ & 3,5-dinitrobenzyl alcohol & $\begin{array}{l}\mathrm{N}(=\mathrm{O})(=\mathrm{O}) \mathrm{c} 1 \mathrm{cc}(\mathrm{N}(=\mathrm{O})=\mathrm{O}) \operatorname{cc}(\mathrm{c} 1) \mathrm{C} \\
\mathrm{O}\end{array}$ & 0.53 & 3 \\
\hline 312 & $\mathrm{p}$ & $100-17-4$ & 4-nitroanisole & $\mathrm{N}(=\mathrm{O})(=\mathrm{O}) \mathrm{c} 1 \mathrm{ccc}(\mathrm{cc} 1) \mathrm{OC}$ & 0.54 & 2,3 \\
\hline 313 & $\mathrm{p}$ & $619-72-7$ & 4-nitrobenzonitrile & $\mathrm{N}(=\mathrm{O})(=\mathrm{O}) \mathrm{c} 1 \operatorname{ccc}(\mathrm{cc} 1) \mathrm{CHN}$ & 0.57 & 2 \\
\hline 314 & $\mathrm{p}$ & $120-47-8$ & ethyl-4-hydroxybenzoate & $\mathrm{C}(\mathrm{OCC})(=\mathrm{O}) \mathrm{c} 1 \mathrm{ccc}(\mathrm{cc} 1) \mathrm{O}$ & 0.57 & $2,3,4,5,7,8,11$ \\
\hline 315 & $\mathrm{p}$ & $119-33-5$ & 4-methyl-2-nitrophenol & $\mathrm{N}(=\mathrm{O})(=\mathrm{O}) \mathrm{c} 1 \mathrm{c}(\mathrm{O}) \operatorname{ccc}(\mathrm{c} 1) \mathrm{C}$ & 0.57 & $3,4,5,7,8,11$ \\
\hline 316 & $\mathrm{p}$ & $6627-55-0$ & 2-bromo-4-methylphenol & $\operatorname{Brc1c}(\mathrm{O}) \operatorname{ccc}(\mathrm{c} 1) \mathrm{C}$ & 0.60 & $2,3,4,5,7,8,11$ \\
\hline 317 & $\mathrm{p}$ & $367-27-1$ & 2,4-difluorophenol & $\mathrm{Fclc}(\mathrm{O}) \operatorname{ccc}(\mathrm{c} 1) \mathrm{F}$ & 0.60 & $2,3,4,5,7,8,11$ \\
\hline 318 & $\mathrm{p}$ & $618-45-1$ & 3-isopropylphenol & $\mathrm{C}(\mathrm{C})(\mathrm{C}) \mathrm{c} 1 \mathrm{cc}(\mathrm{O}) \operatorname{ccc} 1$ & 0.61 & $2,3,4,5,7,8,11$ \\
\hline 319 & $\mathrm{p}$ & $402-45-9$ & $\alpha, \alpha, \alpha$-trifluoro-4-cresol & $\mathrm{C}(\mathrm{F})(\mathrm{F})(\mathrm{F}) \mathrm{c} 1 \mathrm{ccc}(\mathrm{cc} 1) \mathrm{O}$ & 0.62 & 2,4 \\
\hline 320 & $\mathrm{p}$ & $618-80-4$ & 2,6-dichloro-4-nitrophenol & $\mathrm{N}(=\mathrm{O})(=\mathrm{O}) \mathrm{clcc}(\mathrm{Cl}) \mathrm{c}(\mathrm{c}(\mathrm{cl}) \mathrm{Cl}) \mathrm{O}$ & 0.63 & $3,4,5,7,8,11$ \\
\hline 321 & $\mathrm{p}$ & $104-91-6$ & 4-nitrosophenol & $\mathrm{N}(=\mathrm{O}) \mathrm{c} 1 \mathrm{ccc}(\mathrm{cc} 1) \mathrm{O}$ & 0.65 & $2,3,4,5,7,8,11$ \\
\hline 322 & $\mathrm{p}$ & $106-41-2$ & 4-bromophenol & $\mathrm{Brc} 1 \mathrm{ccc}(\mathrm{cc} 1) \mathrm{O}$ & 0.68 & $3,4,5,7,8,11$ \\
\hline 323 & $\mathrm{p}$ & $1129-37-9$ & 4-nitrobenzaldoxime & $\mathrm{N}(=\mathrm{O})(=\mathrm{O}) \mathrm{c} 1 \mathrm{ccc}(\operatorname{cc} 1) \backslash \mathrm{C}=\mathrm{N} \backslash \mathrm{O}$ & 0.68 & 2,3 \\
\hline 324 & $\mathrm{p}$ & $1124-04-5$ & 2-chloro-4,5-dimethylphenol & $\mathrm{Cc} 1 \mathrm{c}(\mathrm{C}) \mathrm{cc}(\mathrm{c}(\mathrm{c} 1) \mathrm{Cl}) \mathrm{O}$ & 0.69 & $3,4,5,7,8,11$ \\
\hline 325 & $\mathrm{p}$ & $86-53-3$ & 1-cyanonaphthalene & $\mathrm{C}(\# \mathrm{~N}) \mathrm{c} 1 \mathrm{c} 2 \mathrm{c}(\mathrm{ccc} 1) \operatorname{ccc} 2$ & 0.69 & 2 \\
\hline 326 & $\mathrm{p}$ & $1570-64-5$ & 4-chloro-2-methylphenol & $\mathrm{Cc} 1 \mathrm{c}(\mathrm{O}) \operatorname{ccc}(\mathrm{c} 1) \mathrm{Cl}$ & 0.70 & $3,4,5,7,8,11$ \\
\hline 327 & $\mathrm{p}$ & $122-94-1$ & 4-butoxyphenol & $\mathrm{O}(\mathrm{CCCC}) \mathrm{c} 1 \mathrm{ccc}(\mathrm{cc} 1) \mathrm{O}$ & 0.70 & $3,4,5,7,8,11$ \\
\hline 328 & $\mathrm{p}$ & $99-77-4$ & Ethyl-4-nitrobenzoate & $\mathrm{C}(\mathrm{OCC})(=\mathrm{O}) \operatorname{c} 1 \operatorname{ccc}(\operatorname{cc} 1) \mathrm{N}(=\mathrm{O})=\mathrm{O}$ & 0.71 & 2 \\
\hline 329 & $\mathrm{p}$ & $36436-65-4$ & 2-hydroxy-4,5-dimethylacetophenone & $\mathrm{C}(\mathrm{C})(=\mathrm{O}) \mathrm{c} 1 \mathrm{c}(\mathrm{O}) \mathrm{cc}(\mathrm{c}(\mathrm{c} 1) \mathrm{C}) \mathrm{C}$ & 0.71 & $3,4,5,7,8$ \\
\hline 330 & $\mathrm{p}$ & $585-34-2$ & 3-tert-butylphenol & $\mathrm{C}(\mathrm{C})(\mathrm{C})(\mathrm{C}) \mathrm{c} 1 \mathrm{cc}(\mathrm{O}) \mathrm{ccc} 1$ & 0.73 & $2,3,4,8,10$ \\
\hline 331 & $\mathrm{p}$ & $2042-14-0$ & 4-methyl-3-nitrophenol & $\mathrm{N}(=\mathrm{O})(=\mathrm{O}) \mathrm{c} 1 \mathrm{c}(\mathrm{C}) \operatorname{ccc}(\mathrm{c} 1) \mathrm{O}$ & 0.74 & $3,4,5,7,8,11$ \\
\hline
\end{tabular}




\begin{tabular}{|c|c|c|c|c|c|c|}
\hline 332 & $\mathrm{p}$ & $87-65-0$ & 2,6-dichlorophenol & $\mathrm{Oc} 1 \mathrm{c}(\mathrm{Cl}) \operatorname{cccc} 1 \mathrm{Cl}$ & 0.74 & $3,4,5,7,8,11$ \\
\hline 333 & $\mathrm{p}$ & 2973-19-5 & 2-chloromethyl-4-nitrophenol & $\mathrm{C}(\mathrm{Cl}) \operatorname{c} 1 \mathrm{cc}(\mathrm{N}(=\mathrm{O})=\mathrm{O}) \operatorname{ccc} 1 \mathrm{O}$ & 0.75 & $3,4,7,8,11$ \\
\hline 334 & $\mathrm{p}$ & $95-85-2$ & 2-amino-4-chlorophenol & $\mathrm{Nc} 1 \mathrm{c}(\mathrm{O}) \operatorname{ccc}(\mathrm{c} 1) \mathrm{Cl}$ & 0.78 & $3,4,8$ \\
\hline 335 & $\mathrm{p}$ & $88-69-7$ & 2-isopropylphenol & $\mathrm{C}(\mathrm{C})(\mathrm{C}) \mathrm{c} 1 \mathrm{c}(\mathrm{O}) \mathrm{cccc} 1$ & 0.80 & $2,3,4,5,7,8,11$ \\
\hline 336 & $\mathrm{p}$ & $392-71-2$ & 2,6-dichloro-4-fluorophenol & $\mathrm{Oc} 1 \mathrm{c}(\mathrm{Cl}) \operatorname{cc}(\mathrm{cc} 1 \mathrm{Cl}) \mathrm{F}$ & 0.80 & $2,3,4,5,7,8,11$ \\
\hline 337 & $\mathrm{p}$ & $100-12-9$ & 4-ethylnitrobenene & $\mathrm{N}(=\mathrm{O})(=\mathrm{O}) \mathrm{c} 1 \mathrm{ccc}(\operatorname{cc} 1) \mathrm{CC}$ & 0.80 & 2,3 \\
\hline 338 & $\mathrm{p}$ & $35421-08-0$ & 4-chloro-3-methylphenol & $\mathrm{C}(\mathrm{Cl}) \mathrm{c} 1 \mathrm{ccc}(\mathrm{cc} 1) \mathrm{O}$ & 0.80 & 2 \\
\hline 339 & $\mathrm{p}$ & $119-34-6$ & 4-amino-2-nitrophenol & $\mathrm{N}(=\mathrm{O})(=\mathrm{O}) \mathrm{c} 1 \mathrm{c}(\mathrm{O}) \operatorname{ccc}(\mathrm{c} 1) \mathrm{N}$ & 0.88 & $3,4,5,7,8,11$ \\
\hline 340 & $\mathrm{p}$ & $95-55-6$ & 2-aminophenol & $\mathrm{Nc1c}(\mathrm{O}) \mathrm{cccc} 1$ & 0.94 & $2,3,4,5,7,8,11$ \\
\hline 341 & $\mathrm{p}$ & $89-72-5$ & 2-sec-butylphenol & $\mathrm{C}([\mathrm{C} @ \mathrm{H}](\mathrm{C}) \mathrm{c} 1 \mathrm{c}(\mathrm{O}) \mathrm{cccc} 1) \mathrm{C}$ & 0.98 & 3 \\
\hline 342 & $\mathrm{p}$ & $14143-32-9$ & 4-chloro-3-ethylphenol & $\mathrm{C}(\mathrm{C}) \mathrm{c} 1 \mathrm{c}(\mathrm{Cl}) \operatorname{ccc}(\mathrm{c} 1) \mathrm{O}$ & 1.08 & $3,4,5,7,8,11$ \\
\hline 343 & $\mathrm{p}$ & $612-24-8$ & 2-nitrobenzonitrile & $\mathrm{C}(\# \mathrm{~N}) \mathrm{c} 1 \mathrm{c}(\mathrm{N}(=\mathrm{O})=\mathrm{O}) \mathrm{cccc} 1$ & 1.08 & 2,3 \\
\hline 344 & $\mathrm{p}$ & $626-02-8$ & 3-iodophenol & Ic1cc(O) $\operatorname{ccc} 1$ & 1.12 & $2,3,4,5,7,8,11$ \\
\hline 345 & $\mathrm{p}$ & $446-36-6$ & 5-fluoro-2-nitrophenol & $\mathrm{N}(=\mathrm{O})(=\mathrm{O}) \mathrm{c} 1 \mathrm{c}(\mathrm{O}) \mathrm{cc}(\mathrm{cc} 1) \mathrm{F}$ & 1.13 & $3,4,5,7,8,11$ \\
\hline 346 & $\mathrm{p}$ & $2613-23-2$ & 3-chloro-4-fluorophenol & $\mathrm{Clc1c}(\mathrm{F}) \operatorname{ccc}(\mathrm{c} 1) \mathrm{O}$ & 1.13 & $2,3,4,5,7,8,11$ \\
\hline 347 & $\mathrm{p}$ & $583-78-8$ & 2,5-dichlorophenol & $\mathrm{Oc} 1 \mathrm{c}(\mathrm{Cl}) \operatorname{ccc}(\mathrm{c} 1) \mathrm{Cl}$ & 1.13 & $3,4,5,7,8,11$ \\
\hline 348 & $\mathrm{p}$ & $591-20-8$ & 3-bromophenol & $\operatorname{Brc1cc}(\mathrm{O}) \operatorname{ccc} 1$ & 1.14 & $3,4,5,7,8,11$ \\
\hline 349 & $\mathrm{p}$ & $769-39-1$ & 2,3,5,6-tetrafluorophenol & $\mathrm{Fc1c}(\mathrm{O}) \mathrm{c}(\mathrm{F}) \mathrm{c}(\mathrm{cc} 1 \mathrm{~F}) \mathrm{F}$ & 1.17 & $2,3,4,5,8,11$ \\
\hline 350 & $\mathrm{p}$ & $6358-07-2$ & 2-amino-4-chloro-5-nitrophenol & $\mathrm{N}(=\mathrm{O})(=\mathrm{O}) \mathrm{c} 1 \mathrm{c}(\mathrm{Cl}) \mathrm{cc}(\mathrm{c}(\mathrm{c} 1) \mathrm{O}) \mathrm{N}$ & 1.17 & $3,4,5,7,8,11$ \\
\hline 351 & $\mathrm{p}$ & $609-93-8$ & 2,6-dinitro-4-cresol & $\begin{array}{l}\mathrm{N}(=\mathrm{O})(=\mathrm{O}) \mathrm{c} 1 \mathrm{c}(\mathrm{O}) \mathrm{c}(\mathrm{N}(=\mathrm{O})=\mathrm{O}) \mathrm{cc}(\mathrm{c} 1 \\
) \mathrm{C}\end{array}$ & 1.23 & $2,3,4,5,7,8,11$ \\
\hline 352 & $\mathrm{p}$ & $7463-51-6$ & 4-bromo-3,5-dimethylphenol & $\operatorname{Brclc}(\mathrm{C}) \operatorname{cc}(\operatorname{cc} 1 \mathrm{C}) \mathrm{O}$ & 1.27 & $3,4,5,7,8,11$ \\
\hline 353 & $\mathrm{p}$ & $576-24-9$ & 2,3-dichlorophenol & $\mathrm{Clc1c}(\mathrm{Cl}) \operatorname{cccc} 1 \mathrm{O}$ & 1.28 & $2,3,4,5,7,8,11$ \\
\hline 354 & $\mathrm{p}$ & $1518-83-8$ & 4-cyclopentylphenol & Oc1cce(cc1)C1CCCC1 & 1.29 & $2,3,4,5,7,8,11$ \\
\hline 355 & $\mathrm{p}$ & $2409-55-4$ & 2-tert-butyl-4-methylphenol & $\mathrm{C}(\mathrm{C})(\mathrm{C})(\mathrm{C}) \mathrm{c} 1 \mathrm{c}(\mathrm{O}) \operatorname{ccc}(\mathrm{c} 1) \mathrm{C}$ & 1.30 & $2,3,4,8$ \\
\hline 356 & $\mathrm{p}$ & $88-18-6$ & 2-tert-butylphenol & $\mathrm{C}(\mathrm{C})(\mathrm{C})(\mathrm{C}) \mathrm{c} 1 \mathrm{c}(\mathrm{O}) \mathrm{cccc} 1$ & 1.30 & $3,4,8$ \\
\hline 357 & $\mathrm{p}$ & $86-00-0$ & 2-nitrobiphenyl & $\mathrm{N}(=\mathrm{O})(=\mathrm{O}) \mathrm{c} 1 \mathrm{c}(\operatorname{ccc} 1) \mathrm{c} 1 \mathrm{ccccc} 1$ & 1.30 & 2,3 \\
\hline 358 & $\mathrm{p}$ & $99-28-5$ & 2,6-dibromo-4-nitrophenol & $\mathrm{N}(=\mathrm{O})(=\mathrm{O}) \mathrm{c} 1 \mathrm{cc}(\mathrm{Br}) \mathrm{c}(\mathrm{c}(\mathrm{c} 1) \mathrm{Br}) \mathrm{O}$ & 1.35 & $2,4,5,7,8,11$ \\
\hline 359 & $\mathrm{p}$ & $3460-18-2$ & 2,5-dibromonitrobenzene & $\mathrm{N}(=\mathrm{O})(=\mathrm{O}) \mathrm{c} 1 \mathrm{c}(\mathrm{Br}) \operatorname{ccc}(\mathrm{c} 1) \mathrm{Br}$ & 1.37 & \\
\hline 360 & $\mathrm{p}$ & $100-02-7$ & 4-nitrophenol & $\mathrm{N}(=\mathrm{O})(=\mathrm{O}) \mathrm{c} 1 \mathrm{ccc}(\mathrm{cc} 1) \mathrm{O}$ & 1.42 & $3,4,5,7,8,11,14$ \\
\hline 361 & $\mathrm{p}$ & $2113-58-8$ & 3-nitrobiphenyl & $\mathrm{N}(=\mathrm{O})(=\mathrm{O}) \mathrm{c} 1 \mathrm{cc}(\mathrm{ccc} 1) \mathrm{c} 1 \mathrm{ccccc} 1$ & 1.57 & 2,3 \\
\hline 362 & $\mathrm{p}$ & $619-08-9$ & 2-chloro-4-nitrophenol & $\mathrm{N}(=\mathrm{O})(=\mathrm{O}) \mathrm{c} 1 \mathrm{cc}(\mathrm{Cl}) \mathrm{c}(\mathrm{cc} 1) \mathrm{O}$ & 1.59 & $3,4,5,7,8,11$ \\
\hline 363 & $\mathrm{p}$ & $1138-52-9$ & 3,5-di-tert-butylphenol & $\mathrm{C}(\mathrm{C})(\mathrm{C})(\mathrm{C}) \mathrm{c} 1 \mathrm{cc}(\mathrm{C}(\mathrm{C})(\mathrm{C}) \mathrm{C}) \mathrm{cc}(\mathrm{c} 1) \mathrm{O}$ & 1.64 & $3,4,8$ \\
\hline 364 & $\mathrm{p}$ & $18979-55-0$ & 4-hexyloxyphenol & $\mathrm{O}(\mathrm{CCCCCC}) \mathrm{c} 1 \mathrm{ccc}(\mathrm{cc} 1) \mathrm{O}$ & 1.64 & $2,3,4,5,7,8,11$ \\
\hline 365 & $\mathrm{p}$ & $2581-34-2$ & 3-methyl-4-nitrophenol & $\mathrm{N}(=\mathrm{O})(=\mathrm{O}) \mathrm{c} 1 \mathrm{c}(\mathrm{C}) \mathrm{cc}(\mathrm{cc} 1) \mathrm{O}$ & 1.73 & $3,4,5,7,8,11$ \\
\hline 366 & $\mathrm{p}$ & $95-77-2$ & 3,4-dichlorophenol & $\mathrm{Clc1c}(\mathrm{Cl}) \operatorname{ccc}(\mathrm{c} 1) \mathrm{O}$ & 1.75 & $2,3,4,5,7,8,11$ \\
\hline 367 & $\mathrm{p}$ & $3217-15-0$ & 4-bromo-2,6-dichlorophenol & $\mathrm{Oc} 1 \mathrm{c}(\mathrm{Cl}) \mathrm{cc}(\mathrm{cclCl}) \mathrm{Br}$ & 1.78 & $2,3,4,5,7,8,11$ \\
\hline 368 & $\mathrm{p}$ & $128-37-0$ & 2,6-di-tert-butyl-4-methylphenol & $\begin{array}{l}\mathrm{C}(\mathrm{C})(\mathrm{C})(\mathrm{C}) \mathrm{c} 1 \mathrm{c}(\mathrm{O}) \mathrm{c}(\mathrm{C}(\mathrm{C})(\mathrm{C}) \mathrm{C}) \mathrm{cc}(\mathrm{c} 1 \\
) \mathrm{C}\end{array}$ & 1.80 & $4,5,7,14$ \\
\hline
\end{tabular}




\begin{tabular}{|c|c|c|c|c|c|c|}
\hline 369 & $\mathrm{p}$ & $89-68-9$ & 4-chloro-2-isopropyl-5-methylphenol & $\mathrm{C}(\mathrm{C})(\mathrm{C}) \mathrm{c} 1 \mathrm{c}(\mathrm{O}) \mathrm{cc}(\mathrm{c}(\mathrm{c} 1) \mathrm{Cl}) \mathrm{C}$ & 1.85 & $3,4,5,7,8,11$ \\
\hline 370 & $\mathrm{p}$ & $836-30-6$ & 4-nitrodiphenylamine & $\mathrm{N}(\mathrm{c} 1 \mathrm{ccc}(\operatorname{cc} 1) \mathrm{N}(=\mathrm{O})=\mathrm{O}) \mathrm{c} 1 \operatorname{ccccc} 1$ & 1.89 & 2,3 \\
\hline 371 & $\mathrm{p}$ & $13037-86-0$ & 4-heptyloxyphenol & $\mathrm{O}(\mathrm{CCCCCCC}) \mathrm{c} 1 \mathrm{ccc}(\mathrm{cc} 1) \mathrm{O}$ & 2.03 & $2,3,4,5,7,8,11$ \\
\hline 372 & $\mathrm{p}$ & $89-64-5$ & 4-chloro-2-nitrophenol & $\mathrm{N}(=\mathrm{O})(=\mathrm{O}) \mathrm{clc}(\mathrm{O}) \operatorname{ccc}(\mathrm{c} 1) \mathrm{Cl}$ & 2.05 & $3,5,7,8,11$ \\
\hline 373 & $\mathrm{p}$ & $140-66-9$ & 4-tert-octylphenol & $\mathrm{C}(\mathrm{CC}(\mathrm{C})(\mathrm{C}) \mathrm{C})(\mathrm{C})(\mathrm{C}) \mathrm{c} 1 \mathrm{ccc}(\mathrm{cc} 1) \mathrm{O}$ & 2.10 & $3,4,8$ \\
\hline 374 & $\mathrm{p}$ & $935-95-5$ & 2,3,5,6-tetrachlorophenol & $\mathrm{Clclc}(\mathrm{O}) \mathrm{c}(\mathrm{Cl}) \mathrm{c}(\mathrm{cclCl}) \mathrm{Cl}$ & 2.22 & $2,3,4,5,8,11,14$ \\
\hline 375 & $\mathrm{p}$ & $933-78-8$ & 2,3,5-trichlorophenol & $\mathrm{Clclc}(\mathrm{Cl}) \operatorname{cc}(\operatorname{cc} 1 \mathrm{O}) \mathrm{Cl}$ & 2.37 & $3,4,5,7,8,11$ \\
\hline 376 & $\mathrm{n}$ & $156-38-7$ & 4-Hydroxyphenylacetic acid & $\mathrm{C}(\mathrm{C}(=\mathrm{O}) \mathrm{O}) \mathrm{c} 1 \mathrm{ccc}(\mathrm{cc} 1) \mathrm{O}$ & -1.50 & $4,5,7,8,11$ \\
\hline 377 & $\mathrm{n}$ & $4383-06-6$ & 3-Hydroxy-4-methoxybenzyl alcohol & $\mathrm{C}(\mathrm{O}) \mathrm{c} 1 \mathrm{cc}(\mathrm{O}) \mathrm{c}(\mathrm{cc} 1) \mathrm{OC}$ & -0.99 & $2,4,5,7,8,11$ \\
\hline 378 & $\mathrm{n}$ & $7149-10-2$ & 4-Hydroxy-3-methoxybenzylamine & $\mathrm{O}(\mathrm{C}) \mathrm{c} 1 \mathrm{cc}(\mathrm{CN}) \operatorname{ccc} 1 \mathrm{O}$ & -0.97 & $2,4,5,7,8,11$ \\
\hline 379 & $\mathrm{n}$ & $498-00-0$ & 4-Hydroxy-3-methoxybenzyl alcohol & $\mathrm{O}(\mathrm{C}) \mathrm{c} 1 \mathrm{cc}(\mathrm{CO}) \operatorname{ccc} 1 \mathrm{O}$ & -0.70 & $2,4,5,7,8,11$ \\
\hline 380 & $\mathrm{n}$ & $90-72-2$ & $\begin{array}{l}\text { 2,4,6-Tris(dimethylaminomethyl) } \\
\text { phenol }\end{array}$ & $\begin{array}{l}\mathrm{C}(\mathrm{N}(\mathrm{C}) \mathrm{C}) \mathrm{c} 1 \mathrm{c}(\mathrm{O}) \mathrm{c}(\mathrm{CN}(\mathrm{C}) \mathrm{C}) \mathrm{cc}(\mathrm{c} 1) \mathrm{C} \\
\mathrm{N}(\mathrm{C}) \mathrm{C}\end{array}$ & -0.52 & $2,4,5,7,8,11$ \\
\hline 381 & $\mathrm{p}$ & $504-15-4$ & 5-Methylresorcinol & $\mathrm{Cc} 1 \mathrm{cc}(\mathrm{O}) \operatorname{cc}(\mathrm{c} 1) \mathrm{O}$ & -0.39 & $4,5,7,8,11,13$ \\
\hline 382 & $\mathrm{p}$ & $14191-95-8$ & 4-Methylcyanophenol & $\mathrm{C}(\mathrm{CHN}) \mathrm{c} 1 \mathrm{ccc}(\mathrm{cc} 1) \mathrm{O}$ & -0.38 & $2,4,5,11$ \\
\hline 383 & $\mathrm{p}$ & $65383-57-5$ & 3-Ethoxy-4-methoxyphenol & $\mathrm{O}(\mathrm{CC}) \mathrm{clc}(\mathrm{OC}) \operatorname{ccc}(\mathrm{c} 1) \mathrm{O}$ & -0.30 & $3,4,5,7,8,11$ \\
\hline 384 & $\mathrm{p}$ & $2380-78-1$ & $\begin{array}{l}\text { 4-Hydroxy-3-methoxyphenethyl } \\
\text { alcohol }\end{array}$ & $\mathrm{O}(\mathrm{C}) \mathrm{c} 1 \mathrm{cc}(\mathrm{CCO}) \operatorname{ccc} 1 \mathrm{O}$ & -0.18 & $4,5,7,8,11$ \\
\hline 385 & $\mathrm{p}$ & $498-02-2$ & 4-Hydroxy-3-methoxyacetophenone & $\mathrm{O}(\mathrm{C}) \mathrm{c} 1 \mathrm{cc}(\mathrm{C}(\mathrm{C})=\mathrm{O}) \operatorname{ccc} 1 \mathrm{O}$ & -0.12 & $4,5,7,8,11$ \\
\hline 386 & $\mathrm{p}$ & $87-28-5$ & 2-Hydroxyethylsalicylate & $\mathrm{C}(\mathrm{OCCO})(=\mathrm{O}) \mathrm{c} 1 \mathrm{c}(\mathrm{O}) \mathrm{cccc} 1$ & -0.08 & $2,4,5,7,8,11$ \\
\hline 387 & $\mathrm{p}$ & $4421-08-3$ & 4-Hydroxy-3-methoxybenzonitrile & $\mathrm{O}(\mathrm{C}) \mathrm{c} 1 \mathrm{cc}(\mathrm{CHN}) \operatorname{ccc} 1 \mathrm{O}$ & -0.03 & 4 \\
\hline 388 & $\mathrm{p}$ & $394-32-1$ & 5-Fluoro-2-hydroxyacetophenone & $\mathrm{C}(\mathrm{C})(=\mathrm{O}) \mathrm{c} 1 \mathrm{c}(\mathrm{O}) \operatorname{ccc}(\mathrm{c} 1) \mathrm{F}$ & 0.04 & $2,4,5,7,8,11$ \\
\hline 389 & $\mathrm{p}$ & $875-59-2$ & 4-Hydroxy-2-methylacetophenone & $\mathrm{C}(\mathrm{C})(=\mathrm{O}) \mathrm{c} 1 \mathrm{c}(\mathrm{C}) \mathrm{cc}(\mathrm{cc} 1) \mathrm{O}$ & 0.19 & 2,4 \\
\hline 390 & $\mathrm{p}$ & $1450-72-2$ & 2-Hydroxy-5-methylacetophenone & $\mathrm{C}(\mathrm{C})(=\mathrm{O}) \mathrm{c} 1 \mathrm{c}(\mathrm{O}) \operatorname{ccc}(\mathrm{c} 1) \mathrm{C}$ & 0.31 & 2,4 \\
\hline 391 & $\mathrm{p}$ & $97-53-0$ & 4-Allyl-2-methoxyphenol & $\mathrm{O}(\mathrm{C}) \mathrm{c} 1 \mathrm{cc}(\mathrm{CC}=\mathrm{C}) \operatorname{ccc} 1 \mathrm{O}$ & 0.42 & $2,4,5,7,8,11$ \\
\hline 392 & $\mathrm{p}$ & $18979-50-5$ & 4-Propyloxyphenol & $\mathrm{O}(\mathrm{CCC}) \mathrm{c} 1 \mathrm{ccc}(\mathrm{cc} 1) \mathrm{O}$ & 0.52 & 4,8 \\
\hline 393 & $\mathrm{p}$ & $700-38-9$ & 5-Methyl-2-nitrophenol & $\mathrm{N}(=\mathrm{O})(=\mathrm{O}) \mathrm{c} 1 \mathrm{c}(\mathrm{O}) \mathrm{cc}(\mathrm{cc} 1) \mathrm{C}$ & 0.59 & 4,8 \\
\hline 394 & $\mathrm{p}$ & $97-54-1$ & 2-Methoxy-4-propenylphenol & $\mathrm{O}(\mathrm{C}) \mathrm{c} 1 \mathrm{cc}(\backslash \mathrm{C}=\mathrm{C} \backslash \mathrm{C}) \operatorname{ccc} 1 \mathrm{O}$ & 0.75 & $4,5,7,8,11$ \\
\hline 395 & $\mathrm{p}$ & $1806-29-7$ & 2,2'-Biphenol & Oc1c(cccc1)c1c(O)cecc1 & 0.88 & 4,8 \\
\hline 396 & $\mathrm{p}$ & $131-55-5$ & 2,2',4,4'-Tetrahydroxybenzophenone & $\begin{array}{l}\mathrm{C}(=\mathrm{O})(\mathrm{c} 1 \mathrm{c}(\mathrm{O}) \mathrm{cc}(\mathrm{cc} 1) \mathrm{O}) \mathrm{c} 1 \mathrm{c}(\mathrm{O}) \mathrm{cc}(\mathrm{cc} \\
1) \mathrm{O}\end{array}$ & 0.96 & 4,8 \\
\hline 397 & $\mathrm{p}$ & $99-71-8$ & 4-Sec-butylphenol & $\mathrm{C}([\mathrm{C} @ \mathrm{H}](\mathrm{C}) \mathrm{c} 1 \mathrm{ccc}(\mathrm{cc} 1) \mathrm{O}) \mathrm{C}$ & 0.98 & $2,4,8$ \\
\hline 398 & $\mathrm{p}$ & $101-18-8$ & 3-Hydroxydiphenylamine & $\mathrm{N}(\mathrm{c} 1 \mathrm{cc}(\mathrm{O}) \operatorname{ccc} 1) \mathrm{c} 1 \mathrm{ccccc} 1$ & 1.01 & 4,8 \\
\hline 399 & $\mathrm{p}$ & $1137-42-4$ & 4-Hydroxybenzophenone & $\mathrm{C}(=\mathrm{O})(\mathrm{c} 1 \mathrm{ccc}(\mathrm{cc} 1) \mathrm{O}) \mathrm{c} 1 \mathrm{ccccc} 1$ & 1.02 & $2,4,5,7,8,11$ \\
\hline 400 & $\mathrm{p}$ & $2437-49-2$ & 2,4,6-Tribromoresorcinol & $\mathrm{Oc} 1 \mathrm{c}(\mathrm{Br}) \mathrm{c}(\mathrm{O}) \mathrm{c}(\mathrm{cc} 1 \mathrm{Br}) \mathrm{Br}$ & 1.06 & $4,5,7,8,11,13$ \\
\hline 401 & $\mathrm{p}$ & $2491-32-9$ & Benzyl-4-hydroxyphenyl ketone & $\mathrm{C}(\mathrm{Cc} 1 \operatorname{ccccc} 1)(=\mathrm{O}) \mathrm{c} 1 \operatorname{ccc}(\mathrm{cc} 1) \mathrm{O}$ & 1.07 & 4,8 \\
\hline 402 & $\mathrm{p}$ & $90-43-7$ & 2-Phenylphenol & Oc1c(c2 $\operatorname{ccc} c 2) \operatorname{ccc} 1$ & 1.09 & $2,4,5,7,8,11$ \\
\hline 403 & $\mathrm{p}$ & $1879-09-0$ & 6-Tert-butyl-2,4-dimethylphenol & $\mathrm{C}(\mathrm{C})(\mathrm{C})(\mathrm{C}) \mathrm{c} 1 \mathrm{c}(\mathrm{O}) \mathrm{c}(\mathrm{C}) \mathrm{cc}(\mathrm{c} 1) \mathrm{C}$ & 1.16 & $3,4,8$ \\
\hline 404 & $\mathrm{p}$ & $117-99-7$ & 2-Hydroxybenzophenone & $\mathrm{C}(=\mathrm{O})(\mathrm{c} 1 \mathrm{c}(\mathrm{O}) \mathrm{cccc} 1) \mathrm{c} 1 \mathrm{ccccc} 1$ & 1.23 & 4,8 \\
\hline 405 & $\mathrm{p}$ & $28994-41-4$ & 2-Hydroxydiphenylmethane & $\mathrm{C}(\mathrm{c} 1 \mathrm{c}(\mathrm{O}) \mathrm{cccc} 1) \mathrm{c} 1 \mathrm{ccccc} 1$ & 1.31 & 4,8 \\
\hline
\end{tabular}




\begin{tabular}{|c|c|c|c|c|c|c|}
\hline 406 & $\mathrm{p}$ & $94-26-8$ & Butyl-4-hydroxybenzoate & $\mathrm{C}(\mathrm{OCCCC})(=\mathrm{O}) \mathrm{c} 1 \mathrm{ccc}(\mathrm{cc} 1) \mathrm{O}$ & 1.33 & 4,8 \\
\hline 407 & $\mathrm{p}$ & $580-51-8$ & 3-Phenylphenol & Oc1ce( $(\operatorname{ccc} 1) \operatorname{cocccc} 1$ & 1.35 & $2,4,5,7,8,11$ \\
\hline 408 & $\mathrm{p}$ & $18979-53-8$ & n-Pentyloxyphenol & $\mathrm{O}(\mathrm{CCCCC}) \mathrm{c} 1 \mathrm{ccc}(\mathrm{cc} 1) \mathrm{O}$ & 1.36 & 4,8 \\
\hline 409 & $\mathrm{p}$ & $131-57-7$ & 2-Hydroxy-4-methoxybenzophenone & $\mathrm{C}(=\mathrm{O})(\mathrm{c} 1 \mathrm{c}(\mathrm{O}) \mathrm{cc}(\mathrm{cc} 1) \mathrm{OC}) \mathrm{c} 1 \mathrm{ccccc} 1$ & 1.42 & $4,5,7,8,11$ \\
\hline 410 & $\mathrm{p}$ & $6521-30-8$ & Isoamyl-4-hydroxybenzoate & $\mathrm{C}(\mathrm{OCCC}(\mathrm{C}) \mathrm{C})(=\mathrm{O}) \mathrm{c} 1 \mathrm{ccc}(\mathrm{cc} 1) \mathrm{O}$ & 1.48 & 4,8 \\
\hline 411 & $\mathrm{p}$ & $90-60-8$ & 3,5-Dichlorosalicylaldehyde & $\mathrm{C}(=\mathrm{O}) \mathrm{clc}(\mathrm{O}) \mathrm{c}(\mathrm{Cl}) \mathrm{cc}(\mathrm{cl}) \mathrm{Cl}$ & 1.55 & 4 \\
\hline 412 & $\mathrm{p}$ & $1131-60-8$ & 4-Cyclohexylphenol & Oc1ccc(cc1)C1CCCCC1 & 1.56 & 4 \\
\hline 413 & $\mathrm{p}$ & $29558-77-8$ & 4-(4-Bromophenyl)phenol & Brc1cce(cc1)c1ccc(cc1)O & 2.31 & 4,8 \\
\hline 414 & $\mathrm{p}$ & $2631-77-8$ & 3,5-Diiododsalicylaldehyde & $\mathrm{C}(=\mathrm{O}) \mathrm{c} 1 \mathrm{c}(\mathrm{O}) \mathrm{c}(\mathrm{I}) \mathrm{cc}(\mathrm{c} 1) \mathrm{I}$ & 2.34 & $4,5,7,8,11$ \\
\hline 415 & $\mathrm{p}$ & $38713-56-3$ & Nonyl-4-hydroxybenzoate & $\begin{array}{l}\mathrm{C}(\mathrm{OCCCCCCCCC})(=\mathrm{O}) \mathrm{c} 1 \mathrm{ccc}(\mathrm{cc} 1) \\
\mathrm{O}\end{array}$ & 2.63 & 4,8 \\
\hline 416 & $\mathrm{p}$ & $320-76-3$ & 4-Bromo-2-.uoro-6-nitrophenol & $\mathrm{N}(=\mathrm{O})(=\mathrm{O}) \mathrm{c} 1 \mathrm{c}(\mathrm{O}) \mathrm{c}(\mathrm{F}) \mathrm{cc}(\mathrm{c} 1) \mathrm{Br}$ & 1.62 & 4,8 \\
\hline 417 & $\mathrm{p}$ & $488-17-5$ & 3-Methylcatechol & Oc1c(C)ecce1O & 0.28 & $4,5,7,8,11,13$ \\
\hline 418 & $\mathrm{p}$ & $452-86-8$ & 4-Methylcatechol & $\mathrm{Oc} 1 \mathrm{c}(\mathrm{O}) \operatorname{ccc}(\mathrm{c} 1) \mathrm{C}$ & 0.37 & $4,5,7,8,11,13$ \\
\hline 419 & $\mathrm{p}$ & $533-73-3$ & 1,2,4-Trihydroxybenzene & $\mathrm{Oc} 1 \mathrm{c}(\mathrm{O}) \operatorname{ccc}(\mathrm{c} 1) \mathrm{O}$ & 0.44 & $4,5,7,8,11,13$ \\
\hline 420 & $\mathrm{p}$ & $123-31-9$ & Hydroquinone & $\mathrm{Oc} 1 \mathrm{ccc}(\mathrm{cc} 1) \mathrm{O}$ & 0.47 & $2,4,5,7,8,11,13$ \\
\hline 421 & $\mathrm{p}$ & $120-80-9$ & Catechol & $\mathrm{Oc} 1 \mathrm{c}(\mathrm{O}) \mathrm{cccc} 1$ & 0.75 & $2,4,5,7,8,11,13,14$ \\
\hline 422 & $\mathrm{p}$ & $87-66-1$ & 1,2,3-Trihydroxybenzene & $\mathrm{Oclc}(\mathrm{O}) \operatorname{cccc} 1 \mathrm{O}$ & 0.85 & $4,5,7,8,11,13$ \\
\hline 423 & $\mathrm{p}$ & $2138-22-9$ & 4-Chlorocatechol & $\mathrm{Oc} 1 \mathrm{c}(\mathrm{O}) \mathrm{ccc}(\mathrm{c} 1) \mathrm{Cl}$ & 1.06 & $2,4,5,7,11,13,14$ \\
\hline 424 & $\mathrm{p}$ & $615-67-8$ & Chlorohydroquinone & $\mathrm{Clc} 1 \mathrm{c}(\mathrm{O}) \operatorname{ccc}(\mathrm{c} 1) \mathrm{O}$ & 1.26 & $4,5,7,8,11,13$ \\
\hline 425 & $\mathrm{p}$ & $2835-96-3$ & 4-Amino-2-cresol & $\mathrm{Cc} 1 \mathrm{c}(\mathrm{O}) \operatorname{ccc}(\mathrm{c} 1) \mathrm{N}$ & 1.31 & $3,4,5,7,8,11$ \\
\hline 426 & $\mathrm{p}$ & $608-43-5$ & 2,3-Dimethylhydroquinone & $\mathrm{Cc} 1 \mathrm{c}(\mathrm{C}) \mathrm{c}(\mathrm{O}) \operatorname{ccc} 1 \mathrm{O}$ & 1.41 & $4,5,7,8,11,13$ \\
\hline 427 & $\mathrm{p}$ & $3096-69-3$ & 4-Amino-2,3-dimethylphenol & $\mathrm{C}[\mathrm{C} @ @ \mathrm{H}] 1 \mathrm{CC}(\mathrm{O} 1)=\mathrm{O}$ & 1.44 & $4,5,7,8,11$ \\
\hline 428 & $\mathrm{p}$ & $583-69-7$ & Bromohydroquinone & $\operatorname{Brclc}(\mathrm{O}) \operatorname{ccc}(\mathrm{c} 1) \mathrm{O}$ & 1.68 & $4,5,7,8,11,13$ \\
\hline 429 & $\mathrm{p}$ & $1198-55-6$ & Tetrachlorocatechol & $\mathrm{c} 1(\mathrm{Cl}) \mathrm{c}(\mathrm{Cl}) \mathrm{c}(\mathrm{Cl}) \mathrm{c}(\mathrm{O}) \mathrm{c}(\mathrm{c} 1 \mathrm{Cl}) \mathrm{O}$ & 1.70 & $2,4,7,8,11,13,14$ \\
\hline 430 & $\mathrm{p}$ & $1079-21-6$ & Phenylhydroquinone & $\mathrm{Oc} 1 \mathrm{c}(\mathrm{cc}(\mathrm{cc} 1) \mathrm{O}) \mathrm{c} 1 \mathrm{ccccc} 1$ & 2.00 & $4,5,7,8,11,13$ \\
\hline 431 & $\mathrm{p}$ & $1020-31-1$ & 3,5-Di-tert-butylcatechol & $\begin{array}{l}\mathrm{C}(\mathrm{C})(\mathrm{C})(\mathrm{C}) \mathrm{c} 1 \mathrm{cc}(\mathrm{C}(\mathrm{C})(\mathrm{C}) \mathrm{C}) \mathrm{cc}(\mathrm{c} 1 \mathrm{O}) \\
\mathrm{O}\end{array}$ & 2.11 & 4,8 \\
\hline 432 & $\mathrm{p}$ & $824-46-4$ & Methoxyhydroquinone & $\mathrm{O}(\mathrm{C}) \mathrm{c} 1 \mathrm{c}(\mathrm{O}) \mathrm{ccc}(\mathrm{c} 1) \mathrm{O}$ & 2.20 & $4,5,7,8,11$ \\
\hline 433 & $\mathrm{p}$ & $704-13-2$ & 3-Hydroxy-4-nitrobenzaldehyde & $\mathrm{N}(=\mathrm{O})(=\mathrm{O}) \mathrm{c} 1 \mathrm{c}(\mathrm{O}) \mathrm{cc}(\mathrm{cc} 1) \mathrm{C}=\mathrm{O}$ & 0.27 & $4,5,7,8,10,11$ \\
\hline 434 & $\mathrm{p}$ & $3011-34-5$ & 4-Hydroxy-3-nitrobenzaldehyde & $\mathrm{N}(=\mathrm{O})(=\mathrm{O}) \mathrm{c} 1 \mathrm{cc}(\mathrm{C}=\mathrm{O}) \operatorname{ccc} 1 \mathrm{O}$ & 0.61 & $4,5,7,8,10,11$ \\
\hline 435 & $\mathrm{p}$ & $601-89-8$ & 2-Nitroresorcinol & $\mathrm{N}(=\mathrm{O})(=\mathrm{O}) \mathrm{c} 1 \mathrm{c}(\mathrm{O}) \operatorname{ccc} 1 \mathrm{O}$ & 0.66 & $4,5,7,8,11,13$ \\
\hline 436 & $\mathrm{p}$ & $3947-58-8$ & $\begin{array}{l}\text { 2-Bromo-2'-hydroxy-5'-nitroacetanilid } \\
\text { e }\end{array}$ & $\mathrm{N}(\mathrm{C}(\mathrm{CBr})=\mathrm{O}) \operatorname{clcc}(\mathrm{N}(=\mathrm{O})=\mathrm{O}) \operatorname{ccc} 1 \mathrm{O}$ & 0.87 & 4,8 \\
\hline 437 & $\mathrm{p}$ & $403-19-0$ & 2-Fluoro-4-nitrophenol & $\mathrm{N}(=\mathrm{O})(=\mathrm{O}) \mathrm{c} 1 \mathrm{cc}(\mathrm{F}) \mathrm{c}(\mathrm{cc} 1) \mathrm{O}$ & 1.07 & 4,8 \\
\hline 438 & $\mathrm{p}$ & $394-33-2$ & 4-Fluoro-2-nitrophenol & $\mathrm{N}(=\mathrm{O})(=\mathrm{O}) \mathrm{c} 1 \mathrm{c}(\mathrm{O}) \operatorname{ccc}(\mathrm{c} 1) \mathrm{F}$ & 1.38 & 4,8 \\
\hline 439 & $\mathrm{p}$ & $7693-52-9$ & 4-Bromo-2-nitrophenol & $\mathrm{N}(=\mathrm{O})(=\mathrm{O}) \mathrm{clc}(\mathrm{O}) \mathrm{ccc}(\mathrm{c} 1) \mathrm{Br}$ & 1.87 & 4,8 \\
\hline 440 & $\mathrm{p}$ & $488-47-1$ & Tetrabromocatechol & $\operatorname{Brclc}(\mathrm{Br}) \mathrm{c}(\mathrm{Br}) \mathrm{c}(\mathrm{c}(\mathrm{c} 1 \mathrm{Br}) \mathrm{O}) \mathrm{O}$ & 0.98 & $4,8,13$ \\
\hline 441 & $\mathrm{p}$ & $527-18-4$ & Tetramethylhydroquinone & $\mathrm{Cc} 1 \mathrm{c}(\mathrm{C}) \mathrm{c}(\mathrm{O}) \mathrm{c}(\mathrm{c}(\mathrm{c} 1 \mathrm{O}) \mathrm{C}) \mathrm{C}$ & 1.28 & $4,8,13$ \\
\hline 442 & $\mathrm{p}$ & $87-87-6$ & Tetrachlorohydroquinone & $\mathrm{Clclc}(\mathrm{Cl}) \mathrm{c}(\mathrm{O}) \mathrm{c}(\mathrm{c}(\mathrm{c} 1 \mathrm{O}) \mathrm{Cl}) \mathrm{Cl}$ & 2.11 & $2,4,8,13$ \\
\hline
\end{tabular}




\begin{tabular}{|c|c|c|c|c|c|c|}
\hline 443 & $\mathrm{n}$ & $108-73-6$ & 1,3,5-Trihydroxybenzene & $\mathrm{Oc} 1 \mathrm{cc}(\mathrm{O}) \mathrm{cc}(\mathrm{c} 1) \mathrm{O}$ & -1.26 & $4,5,7,8,11,13$ \\
\hline 444 & $\mathrm{n}$ & $5471-51-2$ & 4-(4-Hydroxyphenyl)-2-butanone & $\mathrm{C}(\mathrm{CC}(\mathrm{C})=\mathrm{O}) \mathrm{c} 1 \mathrm{ccc}(\mathrm{cc} 1) \mathrm{O}$ & -0.50 & 4,8 \\
\hline 445 & $\mathrm{p}$ & $60563-13-5$ & $\begin{array}{l}\text { Ethyl-4-hydroxy-3-methoxyphenylacet } \\
\text { ate }\end{array}$ & $\mathrm{C}(\mathrm{C}(\mathrm{OCC})=\mathrm{O}) \mathrm{c} 1 \mathrm{cc}(\mathrm{OC}) \mathrm{c}(\mathrm{cc} 1) \mathrm{O}$ & -0.23 & $4,5,7,8,11$ \\
\hline 446 & $\mathrm{p}$ & $95-88-5$ & 4-Chlororesorcinol & $\mathrm{Oclc}(\mathrm{Cl}) \operatorname{ccc}(\mathrm{c} 1) \mathrm{O}$ & 0.13 & $4,5,7,8,11,13$ \\
\hline 447 & $\mathrm{p}$ & $4920-77-8$ & 3-Methyl-2-nitrophenol & $\mathrm{N}(=\mathrm{O})(=\mathrm{O}) \mathrm{c} 1 \mathrm{c}(\mathrm{C}) \operatorname{ccc} 1 \mathrm{O}$ & 0.61 & 4,8 \\
\hline 448 & $\mathrm{p}$ & $5460-31-1$ & 2-Methyl-3-nitrophenol & $\mathrm{N}(=\mathrm{O})(=\mathrm{O}) \mathrm{c} 1 \mathrm{c}(\mathrm{C}) \mathrm{c}(\mathrm{O}) \mathrm{ccc} 1$ & 0.78 & 4,8 \\
\hline 449 & $\mathrm{p}$ & $137-19-9$ & 4,6-Dichlororesorcinol & $\mathrm{Clc1c}(\mathrm{O}) \operatorname{cc}(\mathrm{c}(\mathrm{c} 1) \mathrm{Cl}) \mathrm{O}$ & 0.97 & $2,4,5,7,8,11,13$ \\
\hline 450 & $\mathrm{p}$ & $103-16-2$ & 4-Benzyloxyphenol & $\mathrm{O}(\mathrm{Cc} 1 \mathrm{ccccc} 1) \mathrm{c} 1 \mathrm{ccc}(\mathrm{cc} 1) \mathrm{O}$ & 1.04 & $2,4,5,7,8,11$ \\
\hline 451 & $\mathrm{p}$ & $2374-05-2$ & 4-Bromo-2,6-dimethylphenol & & 1.17 & $3,4,5,7,8,11$ \\
\hline 452 & $\mathrm{p}$ & $92-69-3$ & 4-Phenylphenol & Oc1cce $(\operatorname{cc} 1) \operatorname{clcccc} 1$ & 1.39 & $2,4,5,7,8,11,14$ \\
\hline 453 & $\mathrm{p}$ & $94-18-8$ & Benzyl-4-hydroxybenzoate & $\mathrm{C}(\mathrm{OCc} 1 \operatorname{ccccc} 1)(=\mathrm{O}) \operatorname{clccc}(\operatorname{cc} 1) \mathrm{O}$ & 1.55 & 4,8 \\
\hline 454 & $\mathrm{p}$ & $5153-25-3$ & 2-Ethylhexyl-4'-hydroxybenzoate & $\begin{array}{l}\mathrm{C}(\mathrm{OC}[\mathrm{C} @ \mathrm{H}](\mathrm{CCCC}) \mathrm{CC})(=\mathrm{O}) \mathrm{c} 1 \mathrm{ccc} \\
(\mathrm{cc} 1) \mathrm{O}\end{array}$ & 2.51 & 4,8 \\
\hline 455 & $\mathrm{p}$ & $41458-65-5$ & 6-Amino-2,4-dimethylphenol & $\operatorname{Oc} 1 \mathrm{c}(\mathrm{C}) \operatorname{cc}(\operatorname{cc} 1 \mathrm{~N}) \mathrm{C}$ & 0.89 & $3,4,5,7,8,11$ \\
\hline 456 & $\mathrm{p}$ & $700-13-0$ & Trimethylhydroquinone & $\mathrm{Cc} 1 \mathrm{c}(\mathrm{C}) \mathrm{c}(\mathrm{O}) \mathrm{c}(\mathrm{cc} 1 \mathrm{O}) \mathrm{C}$ & 1.34 & $4,5,7,8,11,13$ \\
\hline 457 & $\mathrm{p}$ & $95-71-6$ & Methylhydroquinone & $\mathrm{Cc} 1 \mathrm{c}(\mathrm{O}) \operatorname{ccc}(\mathrm{c} 1) \mathrm{O}$ & 1.86 & $4,5,7,8,1618$ \\
\hline 458 & $\mathrm{p}$ & $394-41-2$ & 3-Fluoro-4-nitrophenol & $\mathrm{N}(=\mathrm{O})(=\mathrm{O}) \mathrm{c} 1 \mathrm{c}(\mathrm{F}) \mathrm{cc}(\mathrm{cc} 1) \mathrm{O}$ & 0.94 & $4,5,7,8,11$ \\
\hline 459 & $\mathrm{p}$ & $771-63-1$ & Tetra.uorohydroquinone & $\mathrm{Fclc}(\mathrm{F}) \mathrm{c}(\mathrm{O}) \mathrm{c}(\mathrm{c}(\mathrm{c} 1 \mathrm{O}) \mathrm{F}) \mathrm{F}$ & 1.84 & $4,8,13$ \\
\hline 460 & $\mathrm{p}$ & $4214-76-0$ & 2-amino-5-nitropyridine & $\mathrm{N}(=\mathrm{O})(=\mathrm{O}) \mathrm{c} 1 \mathrm{ccc}(\mathrm{nc} 1) \mathrm{N}$ & 0.22 & 2 \\
\hline 461 & $\mathrm{p}$ & $446-10-6$ & 4-fluoro-2-nitrotoluene & $\mathrm{N}(=\mathrm{O})(=\mathrm{O}) \mathrm{clc}(\mathrm{C}) \operatorname{ccc}(\mathrm{c} 1) \mathrm{F}$ & 0.25 & 2 \\
\hline 462 & $\mathrm{p}$ & $607-34-1$ & 5-nitroquinoline & $\mathrm{N}(=\mathrm{O})(=\mathrm{O}) \mathrm{c} 1 \mathrm{c} 2 \mathrm{c}(\mathrm{ccc} 1) \mathrm{nccc} 2$ & 0.39 & 2 \\
\hline 463 & $\mathrm{p}$ & $5428-54-6$ & 2-methyl-5-nitrophenol & $\mathrm{N}(=\mathrm{O})(=\mathrm{O}) \mathrm{c} 1 \mathrm{cc}(\mathrm{O}) \mathrm{c}(\mathrm{cc} 1) \mathrm{C}$ & 0.66 & 2 \\
\hline 464 & $\mathrm{p}$ & $618-87-1$ & 3,5-dinitroaniline & $\mathrm{N}(=\mathrm{O})(=\mathrm{O}) \mathrm{c} 1 \mathrm{cc}(\mathrm{N}(=\mathrm{O})=\mathrm{O}) \operatorname{cc}(\mathrm{c} 1) \mathrm{N}$ & 0.94 & 2 \\
\hline 465 & $\mathrm{p}$ & $607-24-9$ & 2-nitro-1-naphthol & $\mathrm{Oc} 1 \mathrm{c} 2 \mathrm{c}(\operatorname{ccc} 1 \mathrm{~N}(=\mathrm{O})=\mathrm{O}) \operatorname{ccc} 2$ & 1.36 & 2 \\
\hline 466 & $\mathrm{p}$ & $2578-45-2$ & 2-chloro-3,5-dinitropyridine & $\mathrm{N}(=\mathrm{O})(=\mathrm{O}) \mathrm{c} 1 \mathrm{cc}(\mathrm{N}(=\mathrm{O})=\mathrm{O}) \mathrm{cnc} 1 \mathrm{Cl}$ & 2.64 & $3,4,5,7,8,11$ \\
\hline 467 & $\mathrm{n}$ & $67-56-1$ & Methyl alcohol & $\mathrm{CO}$ & -2.666 & 6,12 \\
\hline 468 & $\mathrm{n}$ & $110-63-4$ & 1,4-Butanediol & $\mathrm{C}(\mathrm{CCO}) \mathrm{CO}$ & -2.237 & 6,12 \\
\hline 469 & $\mathrm{n}$ & $6117-80-2$ & cis-2-Butene-1,4-diol & $\mathrm{C}(=\mathrm{C} / \mathrm{CO}) \backslash \mathrm{CO}$ & -2.150 & 6,12 \\
\hline 470 & $\mathrm{n}$ & $64-17-5$ & Ethyl Alcohol & $\mathrm{C}(\mathrm{C}) \mathrm{O}$ & -1.991 & 6,12 \\
\hline 471 & $\mathrm{n}$ & $107-12-0$ & Propionitrile & $\mathrm{C}(\mathrm{C} \# \mathrm{~N}) \mathrm{C}$ & -1.972 & 6 \\
\hline 472 & $\mathrm{n}$ & $107-41-5$ & 2-Methyl-2,4-pentanediol & $\mathrm{C}(\mathrm{C}(\mathrm{C})(\mathrm{C}) \mathrm{O})[\mathrm{C} @ \mathrm{H}](\mathrm{C}) \mathrm{O}$ & -1.953 & 6,12 \\
\hline 473 & $\mathrm{n}$ & $124-68-5$ & 2-Amino-2-methyl-1-propanol & $\mathrm{C}(\mathrm{CO})(\mathrm{C})(\mathrm{C}) \mathrm{N}$ & -1.932 & 6 \\
\hline 474 & $\mathrm{n}$ & $110-65-6$ & butynediol & $\mathrm{C}(\mathrm{\# CCO}) \mathrm{CO}$ & -1.884 & 6,14 \\
\hline 475 & $\mathrm{n}$ & $927-74-2$ & 3-Butyn-1-ol & $\mathrm{C}(\mathrm{C} \# \mathrm{C}) \mathrm{CO}$ & -1.839 & 6,14 \\
\hline 476 & $\mathrm{n}$ & $109-83-1$ & 2-Methylaminoethanol & $\mathrm{C}(\mathrm{CO}) \mathrm{NC}$ & -1.820 & 6,12 \\
\hline 477 & $\mathrm{n}$ & $109-85-3$ & 2-Methoxyethylamine & $\mathrm{C}(\mathrm{CN}) \mathrm{OC}$ & -1.790 & 6,12 \\
\hline 478 & $\mathrm{n}$ & $102-71-6$ & Triethanolamine & $\mathrm{N}(\mathrm{CCO})(\mathrm{CCO}) \mathrm{CCO}$ & -1.749 & 6,12 \\
\hline 479 & $\mathrm{n}$ & $78-93-3$ & 2-Butanone & $\mathrm{C}(\mathrm{CC})(\mathrm{C})=\mathrm{O}$ & -1.746 & 6,12 \\
\hline
\end{tabular}




\begin{tabular}{|c|c|c|c|c|c|c|}
\hline 480 & $\mathrm{n}$ & $6291-85-6$ & 3-Ethoxypropylamine & $\mathrm{C}(\mathrm{CCN}) \mathrm{OCC}$ & -1.703 & 6,12 \\
\hline 481 & $\mathrm{n}$ & $16369-21-4$ & 2-(Propylamino)ethanol & $\mathrm{N}(\mathrm{CCC}) \mathrm{CCO}$ & -1.684 & 6 \\
\hline 482 & $\mathrm{n}$ & $637-88-7$ & 1,4-Cyclohexanedione & $\mathrm{O}=\mathrm{C} 1 \mathrm{CCC}(\mathrm{CC} 1)=\mathrm{O}$ & -1.668 & 6 \\
\hline 483 & $\mathrm{n}$ & $150-90-3$ & Succinic acid, disodium salt & $\mathrm{C}(\mathrm{CC}(=\mathrm{O}) \mathrm{O}) \mathrm{C}(=\mathrm{O}) \mathrm{O}$ & -1.651 & 6 \\
\hline 484 & $\mathrm{n}$ & $110-73-6$ & 2-Ethylaminoethanol & $\mathrm{C}(\mathrm{NCC}) \mathrm{CO}$ & -1.649 & 6,12 \\
\hline 485 & $\mathrm{n}$ & $2117-11-5$ & (+/-)-4-Pentyn-2-ol & $\mathrm{C}([\mathrm{C} @ \mathrm{H}](\mathrm{C}) \mathrm{O}) \mathrm{C} \# \mathrm{C}$ & -1.632 & 6,14 \\
\hline 486 & $\mathrm{n}$ & $78-82-0$ & Isobutyronitrile & $\mathrm{C}(\mathrm{CHN})(\mathrm{C}) \mathrm{C}$ & -1.619 & 6 \\
\hline 487 & $\mathrm{n}$ & $554-12-1$ & methyl propionate & $\mathrm{C}(\mathrm{CC})(\mathrm{OC})=\mathrm{O}$ & -1.609 & 6 \\
\hline 488 & $\mathrm{n}$ & $108-21-4$ & Isopropyl Acetate & $\mathrm{O}(\mathrm{C}(\mathrm{C}) \mathrm{C}) \mathrm{C}(\mathrm{C})=\mathrm{O}$ & -1.590 & 6,12 \\
\hline 489 & $\mathrm{n}$ & $26522-85-0$ & Disodium malonate monohydrate & $\mathrm{C}(\mathrm{C}(=\mathrm{O}) \mathrm{O}) \mathrm{C}(=\mathrm{O}) \mathrm{O}$ & -1.578 & 6 \\
\hline 490 & $\mathrm{n}$ & $15892-23-6$ & $( \pm)$-2-Butanol & $\mathrm{C}([\mathrm{C} @ \mathrm{H}](\mathrm{C}) \mathrm{O}) \mathrm{C}$ & -1.542 & 6,12 \\
\hline 491 & $\mathrm{n}$ & $625-31-0$ & 4-Penten-2-ol & $\mathrm{C}([\mathrm{C} @ \mathrm{H}](\mathrm{C}) \mathrm{O}) \mathrm{C}=\mathrm{C}$ & -1.517 & 6 \\
\hline 492 & $\mathrm{n}$ & $100-37-8$ & Diethylaminoethanol & $\mathrm{N}(\mathrm{CCO})(\mathrm{CC}) \mathrm{CC}$ & -1.496 & 6,12 \\
\hline 493 & $\mathrm{n}$ & $127-00-4$ & 1-chloro-2-propanol & $\mathrm{C}([\mathrm{C} @ \mathrm{H}](\mathrm{C}) \mathrm{O}) \mathrm{Cl}$ & -1.492 & 6,12 \\
\hline 494 & $\mathrm{n}$ & $6117-91-5$ & 2-Buten-1-ol & $\mathrm{C}(=\mathrm{C} \backslash \mathrm{C}) \backslash \mathrm{CO}$ & -1.472 & 6,12 \\
\hline 495 & $\mathrm{n}$ & $109-74-0$ & butyronitrile & $\mathrm{C}(\mathrm{CC}) \mathrm{C \# N}$ & -1.441 & 6 \\
\hline 496 & $\mathrm{n}$ & $5390-04-5$ & 4-Pentyn-1-ol & $\mathrm{C}(\mathrm{CCO}) \mathrm{C} \# \mathrm{C}$ & -1.420 & 6,12 \\
\hline 497 & $\mathrm{n}$ & $19780-84-8$ & 5-Hexyn-3-ol & $\mathrm{C}([\mathrm{C} @ @ \mathrm{H}](\mathrm{CC}) \mathrm{O}) \mathrm{C} \# \mathrm{C}$ & -1.404 & 6,12 \\
\hline 498 & $\mathrm{n}$ & $1569-50-2$ & 3-Penten-2-ol & $\mathrm{C}(\backslash[\mathrm{C} @ \mathrm{H}](\mathrm{C}) \mathrm{O})=\mathrm{C} \backslash \mathrm{C}$ & -1.401 & 6,12 \\
\hline 499 & $\mathrm{n}$ & $115-18-4$ & 2-Methyl-3-buten-2-ol & $\mathrm{C}(\mathrm{C}=\mathrm{C})(\mathrm{C})(\mathrm{C}) \mathrm{O}$ & -1.389 & 6,12 \\
\hline 500 & $\mathrm{n}$ & $78-83-1$ & 2-Methyl-1-propanol & $\mathrm{C}(\mathrm{CO})(\mathrm{C}) \mathrm{C}$ & -1.372 & 6,12 \\
\hline 501 & $\mathrm{n}$ & $762-75-4$ & Tert-butyl formate & $\mathrm{O}(\mathrm{C}(\mathrm{C})(\mathrm{C}) \mathrm{C}) \mathrm{C}=\mathrm{O}$ & -1.372 & 6,12 \\
\hline 502 & $\mathrm{n}$ & $591-78-6$ & 2-Hexanone & $\mathrm{C}(\mathrm{C}(\mathrm{C})=\mathrm{O}) \mathrm{CCC}$ & -1.344 & 6 \\
\hline 503 & $\mathrm{n}$ & $77-75-8$ & 3-Methyl-1-pentyn-3-ol & $\mathrm{C}([\mathrm{C} @](\mathrm{C} \# \mathrm{C})(\mathrm{C}) \mathrm{O}) \mathrm{C}$ & -1.323 & 6,12 \\
\hline 504 & $\mathrm{n}$ & $542-55-2$ & Isobutyl formate & $\mathrm{C}(\mathrm{C}(\mathrm{C}) \mathrm{C}) \mathrm{OC}=\mathrm{O}$ & -1.308 & 6 \\
\hline 505 & $\mathrm{n}$ & $928-90-5$ & 5-Hexyn-1-ol & $\mathrm{C}(\mathrm{CCHC}) \mathrm{CCO}$ & -1.295 & 6,12 \\
\hline 506 & $\mathrm{n}$ & $97-62-1$ & ethyl isobutyrate & $\mathrm{C}(\mathrm{OCC})(\mathrm{C}(\mathrm{C}) \mathrm{C})=\mathrm{O}$ & -1.271 & 6,12 \\
\hline 507 & $\mathrm{n}$ & $502-44-3$ & epsilon-Caprolactone & $\mathrm{O}=\mathrm{C} 1 \mathrm{CCCCCO} 1$ & -1.261 & 6 \\
\hline 508 & $\mathrm{n}$ & $623-42-7$ & Methyl butyrate & $\mathrm{C}(\mathrm{CCC})(\mathrm{OC})=\mathrm{O}$ & -1.246 & 6,12 \\
\hline 509 & $\mathrm{n}$ & $695-06-7$ & Gamma-caprolactone & $\mathrm{C}(\mathrm{C})[\mathrm{C} @ \mathrm{H}] 1 \mathrm{OC}(\mathrm{CC} 1)=\mathrm{O}$ & -1.240 & 6 \\
\hline 510 & $\mathrm{n}$ & $109-60-4$ & n-Propyl acetate & $\mathrm{O}(\mathrm{C}(\mathrm{C})=\mathrm{O}) \mathrm{CCC}$ & -1.238 & 6,12 \\
\hline 511 & $\mathrm{n}$ & $107-87-9$ & 2-Pentanone & $\mathrm{C}(\mathrm{C}(\mathrm{C})=\mathrm{O}) \mathrm{CC}$ & -1.222 & 6,12 \\
\hline 512 & $\mathrm{n}$ & $821-09-0$ & 4-Penten-1-ol & $\mathrm{C}(\mathrm{CCO}) \mathrm{C}=\mathrm{C}$ & -1.215 & 6 \\
\hline 513 & $\mathrm{n}$ & $1068-90-2$ & Diethyl acetamidomalonate & $\begin{array}{l}\mathrm{C}(\mathrm{C}(\mathrm{OCC})=\mathrm{O})(\mathrm{C}(\mathrm{OCC})=\mathrm{O}) \mathrm{NC}(\mathrm{C})= \\
\mathrm{O}\end{array}$ & -1.204 & 6 \\
\hline 514 & $\mathrm{n}$ & $19686-73-8$ & 1-bromo-2-propanol & $\mathrm{C}([\mathrm{C} @ \mathrm{H}](\mathrm{C}) \mathrm{O}) \mathrm{Br}$ & -1.189 & 6 \\
\hline 515 & $\mathrm{n}$ & $4187-86-4$ & 1-Pentyn-3-ol & $\mathrm{C}([\mathrm{C} @ \mathrm{H}](\mathrm{C} \# \mathrm{C}) \mathrm{O}) \mathrm{C}$ & -1.178 & 6,12 \\
\hline 516 & $\mathrm{n}$ & $75-85-4$ & t-Amyl Alcohol & $\mathrm{C}(\mathrm{CC})(\mathrm{C})(\mathrm{C}) \mathrm{O}$ & -1.173 & 6,12 \\
\hline 517 & $\mathrm{n}$ & $1119-40-0$ & Dimethyl Glutarate & $\mathrm{C}(\mathrm{CCC}(\mathrm{OC})=\mathrm{O}) \mathrm{C}(\mathrm{OC})=\mathrm{O}$ & -1.168 & 6 \\
\hline
\end{tabular}




\begin{tabular}{|c|c|c|c|c|c|c|}
\hline 518 & $\mathrm{n}$ & $53955-81-0$ & $\begin{array}{l}\text { Butanoic acid, 2-methyl-, methyl ester } \\
( \pm)\end{array}$ & $\mathrm{C}([\mathrm{C} @ \mathrm{H}](\mathrm{CC}) \mathrm{C})(\mathrm{OC})=\mathrm{O}$ & -1.165 & 6 \\
\hline 519 & $\mathrm{n}$ & $7223-38-3$ & 1-Dimethylamino-2-propyne & $\mathrm{C}(\mathrm{N}(\mathrm{C}) \mathrm{C}) \mathrm{C} \# \mathrm{C}$ & -1.145 & 6,12 \\
\hline 520 & $\mathrm{n}$ & $1576-95-0$ & cis-2-Penten-1-ol & $\mathrm{C}(=\mathrm{C} / \mathrm{CO}) \backslash \mathrm{CC}$ & -1.105 & 6,12 \\
\hline 521 & $\mathrm{n}$ & $96-29-7$ & Ethyl methyl ketone oxime & $\mathrm{C}(\backslash \mathrm{CC})(=\mathrm{N} / \mathrm{O}) \mathrm{C}$ & -1.070 & 6 \\
\hline 522 & $\mathrm{n}$ & $598-32-3$ & 3-Buten-2-ol & $\mathrm{C}([\mathrm{C} @ \mathrm{H}](\mathrm{C}) \mathrm{O})=\mathrm{C}$ & -1.053 & 6,12 \\
\hline 523 & $\mathrm{n}$ & $71-41-0$ & n-Pentanol & $\mathrm{C}(\mathrm{CCO}) \mathrm{CC}$ & -1.030 & 6 \\
\hline 524 & $\mathrm{n}$ & $110-74-7$ & propyl formate & $\mathrm{C}(\mathrm{OC}=\mathrm{O}) \mathrm{CC}$ & -1.022 & 6 \\
\hline 525 & $\mathrm{n}$ & $542-76-7$ & 3-chloropropanenitrile & $\mathrm{C}(\mathrm{CCl}) \mathrm{C} \# \mathrm{~N}$ & -0.999 & 6 \\
\hline 526 & $\mathrm{n}$ & $598-75-4$ & 3-Methyl-2-butanol & $\mathrm{C}([\mathrm{C} @ \mathrm{H}](\mathrm{C}) \mathrm{O})(\mathrm{C}) \mathrm{C}$ & -0.996 & 6,12 \\
\hline 527 & $\mathrm{n}$ & $35161-71-8$ & 2-Propyn-1-amine, N-methyl- & $\mathrm{C}(\mathrm{C} \# \mathrm{C}) \mathrm{NC}$ & -0.982 & 6,12 \\
\hline 528 & $\mathrm{n}$ & $592-35-8$ & O-Butyl carbamate & $\mathrm{O}(\mathrm{CCCC}) \mathrm{C}(\mathrm{N})=\mathrm{O}$ & -0.966 & 6 \\
\hline 529 & $\mathrm{n}$ & $137-32-6$ & 2-Methyl-1-butanol & $\mathrm{C}([\mathrm{C} @ \mathrm{H}](\mathrm{CO}) \mathrm{C}) \mathrm{C}$ & -0.953 & 6,12 \\
\hline 530 & $\mathrm{n}$ & $105-37-3$ & Ethyl Propionate & $\mathrm{C}(\mathrm{OCC})(\mathrm{CC})=\mathrm{O}$ & -0.945 & 6,12 \\
\hline 531 & $\mathrm{n}$ & $592-84-7$ & butyl formate & $\mathrm{C}(\mathrm{CCC}) \mathrm{OC}=\mathrm{O}$ & -0.934 & 6 \\
\hline 532 & $\mathrm{n}$ & $628-20-6$ & 4-Chlorobutyronitrile & $\mathrm{C}(\mathrm{CC} \# \mathrm{~N}) \mathrm{CCl}$ & -0.930 & 6 \\
\hline 533 & $\mathrm{n}$ & $598-56-1$ & N,N-Dimethylethylamine & $\mathrm{N}(\mathrm{CC})(\mathrm{C}) \mathrm{C}$ & -0.908 & 6,12 \\
\hline 534 & $\mathrm{n}$ & $7452-79-1$ & ethyl 2-methylbutyrate & $\mathrm{CC}[\mathrm{CH}](\mathrm{C}(\mathrm{OCC})=\mathrm{O}) \mathrm{C}$ & -0.889 & 6 \\
\hline 535 & $\mathrm{n}$ & $2408-20-0$ & Allyl propionate & $\mathrm{C}(\mathrm{OCC}=\mathrm{C})(\mathrm{CC})=\mathrm{O}$ & -0.879 & 6 \\
\hline 536 & $\mathrm{n}$ & $3240-09-3$ & 5-methyl-5-Hexen-2-one & $\mathrm{C}(\mathrm{CC}(\mathrm{C})=\mathrm{O}) \mathrm{C}(\mathrm{C})=\mathrm{C}$ & -0.875 & 6 \\
\hline 537 & $\mathrm{n}$ & $764-01-2$ & 2-Butyn-1-ol & $\mathrm{C}(\# \mathrm{CC}) \mathrm{CO}$ & -0.868 & 6,14 \\
\hline 538 & $\mathrm{n}$ & $1617-17-0$ & 2-Chloropropanenitrile & $\mathrm{C}([\mathrm{C} @ \mathrm{H}](\mathrm{C}) \mathrm{Cl}) \# \mathrm{~N}$ & -0.863 & 6 \\
\hline 539 & $\mathrm{n}$ & $123-25-1$ & Diethyl Succinate & $\mathrm{C}(\mathrm{CCC}(\mathrm{OCC})=\mathrm{O})(\mathrm{OCC})=\mathrm{O}$ & -0.851 & 6,12 \\
\hline 540 & $\mathrm{n}$ & $624-24-8$ & Methyl pentanoate & $\mathrm{C}(\mathrm{CCCC})(\mathrm{OC})=\mathrm{O}$ & -0.845 & 6,12 \\
\hline 541 & $\mathrm{n}$ & $37143-54-7$ & 1-methoxy-2-propanamine & $\mathrm{C}([\mathrm{C} @ \mathrm{H}](\mathrm{C}) \mathrm{N}) \mathrm{OC}$ & -0.833 & 6 \\
\hline 542 & $\mathrm{n}$ & $106-36-5$ & Propyl propionate & $\mathrm{C}(\mathrm{OCCC})(\mathrm{CC})=\mathrm{O}$ & -0.815 & 6,12 \\
\hline 543 & $\mathrm{n}$ & $4798-44-1$ & 1-Hexen-3-ol & $\mathrm{C}([\mathrm{C} @ @ \mathrm{H}](\mathrm{C}=\mathrm{C}) \mathrm{O}) \mathrm{CC}$ & -0.811 & $6,12,14$ \\
\hline 544 & $\mathrm{n}$ & $627-35-0$ & N-Methylpropylamine & $\mathrm{C}(\mathrm{CC}) \mathrm{NC}$ & -0.809 & 6,12 \\
\hline 545 & $\mathrm{n}$ & $96-23-1$ & 1,3-dichloroisopropanol & $\mathrm{C}(\mathrm{CCl})(\mathrm{CCl}) \mathrm{O}$ & -0.793 & 6 \\
\hline 546 & $\mathrm{n}$ & $638-49-3$ & n-Amyl Formate & $\mathrm{C}(\mathrm{COC}=\mathrm{O}) \mathrm{CCC}$ & -0.783 & 6 \\
\hline 547 & $\mathrm{n}$ & $928-97-2$ & trans-3-Hexen-1-ol & $\mathrm{C}(=\mathrm{C} \backslash \mathrm{CC}) \backslash \mathrm{CCO}$ & -0.777 & 6,12 \\
\hline 548 & $\mathrm{n}$ & $2623-87-2$ & 4-Bromobutyric acid & $\mathrm{C}(\mathrm{C}(=\mathrm{O}) \mathrm{O}) \mathrm{CCBr}$ & -0.771 & 6,12 \\
\hline 549 & $\mathrm{n}$ & $928-51-8$ & 4-chloro-1-butanol & $\mathrm{C}(\mathrm{CCO}) \mathrm{CCl}$ & -0.759 & 6,12 \\
\hline 550 & $\mathrm{n}$ & $616-39-7$ & N-Ethyl-N-methylethanamine & $\mathrm{N}(\mathrm{CC})(\mathrm{CC}) \mathrm{C}$ & -0.756 & 6,12 \\
\hline 551 & $\mathrm{n}$ & $624-95-3$ & 3,3-Dimethyl-1-butanol & $\mathrm{C}(\mathrm{C}(\mathrm{C})(\mathrm{C}) \mathrm{C}) \mathrm{CO}$ & -0.737 & 6,12 \\
\hline 552 & $\mathrm{n}$ & $112245-13-3$ & 2-Amino-3,3-dimethyl-1-butanol & $\mathrm{C}([\mathrm{C} @ @ \mathrm{H}](\mathrm{CO}) \mathrm{N})(\mathrm{C})(\mathrm{C}) \mathrm{C}$ & -0.718 & 6,12 \\
\hline 553 & $\mathrm{n}$ & $141-82-2$ & Malonic acid & $\mathrm{C}(\mathrm{C}(=\mathrm{O}) \mathrm{O}) \mathrm{C}(=\mathrm{O}) \mathrm{O}$ & -0.709 & 6 \\
\hline 554 & $\mathrm{n}$ & $600-36-2$ & 2,4-Dimethyl-3-pentanol & $\mathrm{C}(\mathrm{C}(\mathrm{C}) \mathrm{C})(\mathrm{C}(\mathrm{C}) \mathrm{C}) \mathrm{O}$ & -0.705 & 6,12 \\
\hline 555 & $\mathrm{n}$ & $64-67-5$ & Diethyl Sulfate & $\mathrm{S}(\mathrm{OCC})(\mathrm{OCC})(=\mathrm{O})=\mathrm{O}$ & -0.698 & 6 \\
\hline
\end{tabular}




\begin{tabular}{|c|c|c|c|c|c|c|}
\hline 556 & $\mathrm{n}$ & $2067-33-6$ & 5-Bromovaleric acid & $\mathrm{C}(\mathrm{CCCBr}) \mathrm{C}(=\mathrm{O}) \mathrm{O}$ & -0.693 & 6,12 \\
\hline 557 & $\mathrm{n}$ & $10051-44-2$ & Sodium Caproate & $\mathrm{C}(\mathrm{CCCC}) \mathrm{C}(=\mathrm{O}) \mathrm{O}$ & -0.680 & 6 \\
\hline 558 & $\mathrm{n}$ & $110-68-9$ & N-methylbutylamine & $\mathrm{C}(\mathrm{CNC}) \mathrm{CC}$ & -0.678 & 6,12 \\
\hline 559 & $\mathrm{n}$ & $4146-04-7$ & 2-Amino-1-pentanol & $\mathrm{C}([\mathrm{CH}](\mathrm{N}) \mathrm{CO}) \mathrm{CC}$ & -0.672 & 6,12 \\
\hline 560 & $\mathrm{n}$ & $123-19-3$ & Dipropyl Ketone & $\mathrm{C}(\mathrm{CCC})(\mathrm{CCC})=\mathrm{O}$ & -0.669 & 6,12 \\
\hline 561 & $\mathrm{n}$ & $24629-25-2$ & $\mathrm{~L}(+)$-Isoleucinol & $\mathrm{C}([\mathrm{C} @ @ \mathrm{H}]([\mathrm{C} @ @ \mathrm{H}](\mathrm{CO}) \mathrm{N}) \mathrm{C}) \mathrm{C}$ & -0.659 & 6,12 \\
\hline 562 & $\mathrm{n}$ & $105-38-4$ & Vinyl propionate & $\mathrm{C}(\mathrm{OC}=\mathrm{C})(\mathrm{CC})=\mathrm{O}$ & -0.653 & 6 \\
\hline 563 & $\mathrm{n}$ & $625-38-7$ & Vinylacetic acid & $\mathrm{C}(\mathrm{C}(=\mathrm{O}) \mathrm{O}) \mathrm{C}=\mathrm{C}$ & -0.642 & 6 \\
\hline 564 & $\mathrm{n}$ & $6317-49-3$ & Diethyl 4-oxoheptanedioate & $\begin{array}{l}\mathrm{C}(\mathrm{CCC}(\mathrm{OCC})=\mathrm{O})(\mathrm{CCC}(\mathrm{OCC})=\mathrm{O})= \\
\mathrm{O}\end{array}$ & -0.638 & 6 \\
\hline 565 & $\mathrm{n}$ & $2051-78-7$ & Allyl butyrate & $\mathrm{C}(\mathrm{OCC}=\mathrm{C})(\mathrm{CCC})=\mathrm{O}$ & -0.636 & 6 \\
\hline 566 & $\mathrm{n}$ & $7533-40-6$ & L(+)-Leucinol & $\mathrm{CC}(\mathrm{C}) \mathrm{C}[\mathrm{CH}](\mathrm{N}) \mathrm{CO}$ & -0.619 & 6,12 \\
\hline 567 & $\mathrm{n}$ & $124-04-9$ & Adipic acid & $\mathrm{C}(\mathrm{CCC}(=\mathrm{O}) \mathrm{O}) \mathrm{CC}(=\mathrm{O}) \mathrm{O}$ & -0.606 & 6,12 \\
\hline 568 & $\mathrm{n}$ & $16369-05-4$ & DL-2-Amino-3-methyl-1-butanol & $\mathrm{C}([\mathrm{C} @ \mathrm{H}](\mathrm{CO}) \mathrm{N})(\mathrm{C}) \mathrm{C}$ & -0.585 & 6,12 \\
\hline 569 & $\mathrm{n}$ & $2052-01-9$ & alpha-Bromoisobutyric acid & $\mathrm{C}(\mathrm{C}(=\mathrm{O}) \mathrm{O})(\mathrm{Br})(\mathrm{C}) \mathrm{C}$ & -0.585 & 6,12 \\
\hline 570 & $\mathrm{n}$ & $107-85-7$ & Isoamylamine & $\mathrm{C}(\mathrm{C}(\mathrm{C}) \mathrm{C}) \mathrm{CN}$ & -0.577 & 6,12 \\
\hline 571 & $\mathrm{n}$ & $6261-22-9$ & 2-Pentyn-1-ol & $\mathrm{C}(\mathrm{\# CCO}) \mathrm{CC}$ & -0.572 & 6,12 \\
\hline 572 & $\mathrm{n}$ & $106-70-7$ & methyl caproate & $\mathrm{C}(\mathrm{CCCCC})(\mathrm{OC})=\mathrm{O}$ & -0.561 & 6,12 \\
\hline 573 & $\mathrm{n}$ & $107-54-0$ & 3,5-Dimethyl-1-hexyn-3-ol & $\mathrm{C}([\mathrm{C} @](\mathrm{C} \# \mathrm{C})(\mathrm{C}) \mathrm{O}) \mathrm{C}(\mathrm{C}) \mathrm{C}$ & -0.553 & 6 \\
\hline 574 & $\mathrm{n}$ & $107-93-7$ & trans-Crotonic acid & $\mathrm{C}(\backslash \mathrm{C}(=\mathrm{O}) \mathrm{O})=\mathrm{C} \backslash \mathrm{C}$ & -0.545 & 6,12 \\
\hline 575 & $\mathrm{n}$ & $505-48-6$ & Suberic acid & $\mathrm{C}(\mathrm{CC}(=\mathrm{O}) \mathrm{O}) \mathrm{CCCCC}(=\mathrm{O}) \mathrm{O}$ & -0.512 & 6,12 \\
\hline 576 & $\mathrm{p}$ & $623-71-2$ & Ethyl 3-chloropropiona & $\mathrm{C}(\mathrm{CCCl})(\mathrm{OCC})=\mathrm{O}$ & -0.498 & 6 \\
\hline 577 & $\mathrm{p}$ & $108-22-5$ & Isopropenyl aceta & $\mathrm{O}(\mathrm{C}(\mathrm{C})=\mathrm{C}) \mathrm{C}(\mathrm{C})=\mathrm{O}$ & -0.489 & 6 \\
\hline 578 & $\mathrm{p}$ & $123-86-4$ & Butyl acetate & $\mathrm{O}(\mathrm{CCCC}) \mathrm{C}(\mathrm{C})=\mathrm{O}$ & -0.486 & 6,12 \\
\hline 579 & $\mathrm{p}$ & $123-38-6$ & Propionaldehyde & $\mathrm{C}(\mathrm{C}=\mathrm{O}) \mathrm{C}$ & -0.486 & 6,12 \\
\hline 580 & $\mathrm{p}$ & $\begin{array}{l}\text { 4-Methyl-2-he } \\
\text { ptyn-4-ol }\end{array}$ & 4-Methyl-2-heptyn-4-ol & $\mathrm{C}(\mathrm{C}) \# \mathrm{C}[\mathrm{C} @](\mathrm{C})(\mathrm{O}) \mathrm{CCC}$ & -0.482 & 6 \\
\hline 581 & $\mathrm{p}$ & $123-15-9$ & methyl valeraldehyde & $\mathrm{C}([\mathrm{C} @ \mathrm{H}](\mathrm{C}=\mathrm{O}) \mathrm{C}) \mathrm{CC}$ & -0.475 & 6,12 \\
\hline 582 & $\mathrm{p}$ & $5332-06-9$ & 4-Bromobutyronitrile & $\mathrm{C}(\mathrm{CC} \# \mathrm{~N}) \mathrm{CBr}$ & -0.466 & 6 \\
\hline 583 & $\mathrm{p}$ & $3031-66-1$ & 3-Hexyn-2,5-diol & $\mathrm{C}(\# \mathrm{C}[\mathrm{C} @ \mathrm{H}](\mathrm{C}) \mathrm{O})[\mathrm{C} @ @ \mathrm{H}](\mathrm{C}) \mathrm{O}$ & -0.460 & 6 \\
\hline 584 & $\mathrm{p}$ & $110-93-0$ & 6-Methyl-5-hepten-2-one & $\mathrm{C}(\backslash \mathrm{C}=\mathrm{C}(\backslash \mathrm{C}) \mathrm{C}) \mathrm{CC}(\mathrm{C})=\mathrm{O}$ & -0.452 & 6 \\
\hline 585 & $\mathrm{p}$ & $535-13-7$ & Ethyl 2-chloropropionate & $\mathrm{C}(\mathrm{OCC})([\mathrm{C} @ \mathrm{H}](\mathrm{C}) \mathrm{Cl})=\mathrm{O}$ & -0.431 & 6 \\
\hline 586 & $\mathrm{p}$ & $20487-40-5$ & tert-butyl propionate & $\mathrm{O}(\mathrm{C}(\mathrm{C})(\mathrm{C}) \mathrm{C}) \mathrm{C}(\mathrm{CC})=\mathrm{O}$ & -0.410 & 6,12 \\
\hline 587 & $\mathrm{p}$ & $764-60-3$ & 2-Hexyn-1-ol & $\mathrm{C}(\mathrm{CCC}) \# \mathrm{CCO}$ & -0.384 & 6 \\
\hline 588 & $\mathrm{p}$ & $629-33-4$ & n-Hexyl formate & $\mathrm{C}(\mathrm{CCCC}) \mathrm{COC}=\mathrm{O}$ & -0.382 & 6 \\
\hline 589 & $\mathrm{p}$ & $123-72-8$ & Butyraldehyde & $\mathrm{C}(\mathrm{CC}) \mathrm{C}=\mathrm{O}$ & -0.381 & 6,12 \\
\hline 590 & $\mathrm{p}$ & $111-27-3$ & n-Hexanol & $\mathrm{C}(\mathrm{CCC}) \mathrm{CCO}$ & -0.379 & 6,12 \\
\hline 591 & $\mathrm{p}$ & $53723-18-5$ & 5-Octyn-3-ol & $\mathrm{C}([\mathrm{C} @ \mathrm{H}](\mathrm{CC}) \mathrm{O}) \mathrm{C} \# \mathrm{CCC}$ & -0.376 & 6 \\
\hline 592 & $\mathrm{p}$ & $2987-16-8$ & 3,3-Dimethylbutyraldehyde & $\mathrm{C}(\mathrm{C}(\mathrm{C})(\mathrm{C}) \mathrm{C}) \mathrm{C}=\mathrm{O}$ & -0.374 & 6,12 \\
\hline
\end{tabular}




\begin{tabular}{|c|c|c|c|c|c|c|}
\hline 593 & $\mathrm{p}$ & $638-29-9$ & valeryl chloride & $\mathrm{C}(\mathrm{C}(\mathrm{Cl})=\mathrm{O}) \mathrm{CCC}$ & -0.345 & 6 \\
\hline 594 & $\mathrm{p}$ & $79-31-2$ & isobutyric acid & $\mathrm{C}(\mathrm{C}(=\mathrm{O}) \mathrm{O})(\mathrm{C}) \mathrm{C}$ & -0.333 & 6,12 \\
\hline 595 & $\mathrm{p}$ & 14916-79-1 & 3-Heptyn-1-ol & $\mathrm{C}(\# \mathrm{CCCO}) \mathrm{CCC}$ & -0.323 & 6,12 \\
\hline 596 & $\mathrm{p}$ & $471-25-0$ & Propiolic Acid & $\mathrm{C}(\mathrm{C} \# \mathrm{C})(=\mathrm{O}) \mathrm{O}$ & -0.294 & 6 \\
\hline 597 & $\mathrm{p}$ & $3153-36-4$ & Ethyl 4-chlorobutyrate & $\mathrm{C}(\mathrm{CCCCl})(\mathrm{OCC})=\mathrm{O}$ & -0.281 & 6 \\
\hline 598 & $\mathrm{p}$ & $20515-19-9$ & methyl (E)-3-pentenoate & $\mathrm{C}(\mathrm{C} \backslash \mathrm{C}=\mathrm{C} \backslash \mathrm{C})(\mathrm{OC})=\mathrm{O}$ & -0.276 & 6 \\
\hline 599 & $\mathrm{p}$ & 646-07-1 & Isocaproic Acid & $\mathrm{C}(\mathrm{CC}(=\mathrm{O}) \mathrm{O}) \mathrm{C}(\mathrm{C}) \mathrm{C}$ & -0.272 & 6,12 \\
\hline 600 & $\mathrm{p}$ & $111-20-6$ & Sebacic Acid & $\mathrm{C}(\mathrm{CC}(=\mathrm{O}) \mathrm{O}) \mathrm{CCCCCCC}(=\mathrm{O}) \mathrm{O}$ & -0.268 & 6,12 \\
\hline 601 & $\mathrm{p}$ & $7383-19-9$ & 1-Heptyn-3-ol & $\mathrm{C}([\mathrm{C} @ \mathrm{H}](\mathrm{C} \# \mathrm{C}) \mathrm{O}) \mathrm{CCC}$ & -0.265 & 6,12 \\
\hline 602 & $\mathrm{p}$ & $2168-93-6$ & n-Butyl sulfoxide & $\mathrm{S}(\mathrm{CCCC})(\mathrm{CCCC})=\mathrm{O}$ & -0.257 & 6 \\
\hline 603 & $\mathrm{p}$ & $75-98-9$ & Pivalic Acid & $\mathrm{C}(\mathrm{C}(=\mathrm{O}) \mathrm{O})(\mathrm{C})(\mathrm{C}) \mathrm{C}$ & -0.254 & 6,12 \\
\hline 604 & $\mathrm{p}$ & $629-08-3$ & heptanenitrile & $\mathrm{C}(\mathrm{CCC} \# \mathrm{~N}) \mathrm{CCC}$ & -0.237 & 6 \\
\hline 605 & $\mathrm{p}$ & $1577-18-0$ & trans-3-Hexenoic acid & $\mathrm{C}(\backslash \mathrm{C}=\mathrm{C} \backslash \mathrm{CC}) \mathrm{C}(=\mathrm{O}) \mathrm{O}$ & -0.222 & 6 \\
\hline 606 & $\mathrm{p}$ & $5414-21-1$ & 5-Bromovaleronitrile & $\mathrm{C}(\mathrm{CCBr}) \mathrm{CC} \# \mathrm{~N}$ & -0.215 & 6 \\
\hline 607 & $\mathrm{p}$ & $20739-59-7$ & 4-Hexyn-3-ol & $\mathrm{C}([\mathrm{C} @ \mathrm{H}](\mathrm{CC}) \mathrm{O}) \# \mathrm{CC}$ & -0.195 & 6 \\
\hline 608 & $\mathrm{p}$ & $109-65-9$ & 1-Bromobutane & $\mathrm{C}(\mathrm{CBr}) \mathrm{CC}$ & -0.180 & 6 \\
\hline 609 & $\mathrm{p}$ & $66793-96-2$ & 3-Ethyl-2,2-dimethyl-3-pentanol & $\mathrm{C}(\mathrm{C}(\mathrm{C})(\mathrm{C}) \mathrm{C})(\mathrm{CC})(\mathrm{CC}) \mathrm{O}$ & -0.169 & 6,12 \\
\hline 610 & $\mathrm{p}$ & $111-13-7$ & 2-Octanone & $\mathrm{C}(\mathrm{CC}(\mathrm{C})=\mathrm{O}) \mathrm{CCCC}$ & -0.146 & 6,12 \\
\hline 611 & $\mathrm{p}$ & $13419-69-7$ & trans-2-Hexenoic acid & $\mathrm{C}(=\mathrm{C} \backslash \mathrm{CCC}) \backslash \mathrm{C}(=\mathrm{O}) \mathrm{O}$ & -0.128 & 6,12 \\
\hline 612 & $\mathrm{p}$ & $10031-87-5$ & 2-Ethylbutyl acetate & $\mathrm{C}(\mathrm{COC}(\mathrm{C})=\mathrm{O})(\mathrm{CC}) \mathrm{CC}$ & -0.120 & 6 \\
\hline 613 & $\mathrm{p}$ & $693-23-2$ & Dodecanedioic acid & $\mathrm{C}(\mathrm{CC}(=\mathrm{O}) \mathrm{O}) \mathrm{CCCCCCCCC}(=\mathrm{O}) \mathrm{O}$ & -0.086 & 6,12 \\
\hline 614 & $\mathrm{p}$ & 1984-06-1 & Octanoic acid, sodium salt & $\mathrm{C}(\mathrm{CCCCC}) \mathrm{CC}(=\mathrm{O}) \mathrm{O}$ & -0.080 & 6 \\
\hline 615 & $\mathrm{p}$ & $821-55-6$ & 2-Nonanone & $\mathrm{C}(\mathrm{CCCCC}) \mathrm{CC}(\mathrm{C})=\mathrm{O}$ & 0.660 & 6,12 \\
\hline 616 & $\mathrm{p}$ & $2050-23-9$ & Diethyl suberate & $\mathrm{C}(\mathrm{CCCCCCC}(\mathrm{OCC})=\mathrm{O})(\mathrm{OCC})=\mathrm{O}$ & 0.702 & 6,12 \\
\hline 617 & $\mathrm{p}$ & $142-19-8$ & Allyl heptylate & $\mathrm{C}(\mathrm{C}(\mathrm{OCC}=\mathrm{C})=\mathrm{O}) \mathrm{CCCCC}$ & 0.728 & 6 \\
\hline 618 & $\mathrm{p}$ & $31502-14-4$ & trans-2-Nonen-1-ol & $\mathrm{C}(\mathrm{O}) \backslash \mathrm{C}=\mathrm{C} \backslash \mathrm{CCCCCC}$ & 0.752 & 6 \\
\hline 619 & $\mathrm{p}$ & $533-68-6$ & DL-Ethyl 2-bromobutyrate & $\mathrm{C}([\mathrm{C} @ @ \mathrm{H}](\mathrm{CC}) \mathrm{Br})(\mathrm{OCC})=\mathrm{O}$ & 0.764 & 6 \\
\hline 620 & $\mathrm{p}$ & $105-99-7$ & Dibutyl Adipate & $\begin{array}{l}\mathrm{C}(\mathrm{CCCCC}(\mathrm{OCCCC})=\mathrm{O})(\mathrm{OCCCC})= \\
\mathrm{O}\end{array}$ & 0.792 & 6,12 \\
\hline 621 & $\mathrm{p}$ & $107-14-2$ & chloroacetonitrile & $\mathrm{C}(\mathrm{C} \# \mathrm{~N}) \mathrm{Cl}$ & 0.845 & 6 \\
\hline 622 & $\mathrm{p}$ & $143-08-8$ & Nonyl Alcohol & $\mathrm{C}(\mathrm{CCCCO}) \mathrm{CCCC}$ & 0.855 & 6,12 \\
\hline 623 & $\mathrm{p}$ & $112-37-8$ & n-Hendecanoic Acid & $\mathrm{C}(\mathrm{CCCCCC}) \mathrm{CCCC}(=\mathrm{O}) \mathrm{O}$ & 0.898 & 6,12 \\
\hline 624 & $\mathrm{p}$ & $21661-97-2$ & Cis-7-decen-1-al & $\mathrm{C}(\mathrm{C} \backslash \mathrm{C}=\mathrm{C} / \mathrm{CC}) \mathrm{CCCC}=\mathrm{O}$ & 0.949 & 6,12 \\
\hline 625 & $\mathrm{p}$ & $4117-14-0$ & 2-Decyn-1-ol & $\mathrm{C}(\mathrm{CCCCC}) \mathrm{CC} \# \mathrm{CCO}$ & 0.986 & $6,12,14$ \\
\hline 626 & $\mathrm{p}$ & $106-79-6$ & decanedioic acid dimethyl ester & $\mathrm{C}(\mathrm{CCCCCCCCC}(\mathrm{OC})=\mathrm{O})(\mathrm{OC})=\mathrm{O}$ & 1.011 & 6,12 \\
\hline 627 & $\mathrm{p}$ & $3196-15-4$ & Methyl 2-bromobutyrate & $\mathrm{C}([\mathrm{C} @ \mathrm{H}](\mathrm{CC}) \mathrm{Br})(\mathrm{OC})=\mathrm{O}$ & 1.024 & 6 \\
\hline 628 & $\mathrm{p}$ & $1731-84-6$ & Methyl nonanoate & $\mathrm{C}(\mathrm{CCC}(\mathrm{OC})=\mathrm{O}) \mathrm{CCCCC}$ & 1.042 & 6,12 \\
\hline 629 & $\mathrm{p}$ & $629-03-8$ & 1,6-Dibromohexane & $\mathrm{C}(\mathrm{CCCBr}) \mathrm{CCBr}$ & 1.046 & 6 \\
\hline 630 & $\mathrm{p}$ & 112-14-1 & n-Octyl Acetate & $\mathrm{C}(\mathrm{CCCCCC}) \mathrm{COC}(\mathrm{C})=\mathrm{O}$ & 1.057 & 6,12 \\
\hline
\end{tabular}




\begin{tabular}{|c|c|c|c|c|c|c|}
\hline 631 & $\mathrm{p}$ & $51721-39-2$ & 3-Decyn-1-ol & $\mathrm{C}(\mathrm{CCCCC}) \mathrm{C} \# \mathrm{CCCO}$ & 1.127 & 6 \\
\hline 632 & $\mathrm{p}$ & $624-20-4$ & 1,2-Dibromohexane & $\mathrm{C}([\mathrm{C} @ \mathrm{H}](\mathrm{CBr}) \mathrm{Br}) \mathrm{CCC}$ & 1.146 & 6 \\
\hline 633 & $\mathrm{p}$ & $65405-70-1$ & trans-4-Decen-1-al & $\mathrm{C}(\backslash \mathrm{CCCCC})=\mathrm{C} \backslash \mathrm{CCC}=\mathrm{O}$ & 1.208 & 6,12 \\
\hline 634 & $\mathrm{p}$ & $110-40-7$ & Diethyl Sebacate & $\begin{array}{l}\mathrm{C}(\mathrm{CCCCCCCC}(\mathrm{OCC})=\mathrm{O}) \mathrm{C}(\mathrm{OCC})= \\
\mathrm{O}\end{array}$ & 1.354 & 6,12 \\
\hline 635 & $\mathrm{p}$ & $1731-86-8$ & Undecanoic acid, methyl ester & $\mathrm{C}(\mathrm{CCC}(\mathrm{OC})=\mathrm{O}) \mathrm{CCCCCCC}$ & 1.425 & 6,12 \\
\hline 636 & $\mathrm{p}$ & $1694-29-7$ & 3-Chloro-2,4-pentanedione & $\mathrm{C}(\mathrm{C}(\mathrm{C})=\mathrm{O})(\mathrm{C}(\mathrm{C})=\mathrm{O}) \mathrm{Cl}$ & 1.444 & 6 \\
\hline 637 & $\mathrm{p}$ & $2244-07-7$ & Undecanenitrile & $\mathrm{C}(\mathrm{CCCCC \# N}) \mathrm{CCCCC}$ & 1.650 & 6 \\
\hline 638 & $\mathrm{p}$ & $16090-77-0$ & Octanedioic acid, dibutyl ester & $\begin{array}{l}\mathrm{C}(\mathrm{C}(\mathrm{OCCCC})=\mathrm{O}) \mathrm{CCCCCC}(\mathrm{OCCC} \\
\mathrm{C})=\mathrm{O}\end{array}$ & 1.656 & 6,12 \\
\hline 639 & $\mathrm{p}$ & $112-44-7$ & Undecanal & $\mathrm{C}(\mathrm{CCCCC}=\mathrm{O}) \mathrm{CCCCC}$ & 1.690 & 6 \\
\hline 640 & $\mathrm{p}$ & $112-20-9$ & n-Nonylamine & $\mathrm{C}(\mathrm{CCCCN}) \mathrm{CCCC}$ & 1.701 & 6 \\
\hline 641 & $\mathrm{p}$ & $592-82-5$ & 1-isothiocyanato-butane & $\mathrm{C}(\mathrm{CCC}) \mathrm{N}=\mathrm{C}=\mathrm{S}$ & 1.721 & 6 \\
\hline 642 & $\mathrm{p}$ & $2437-25-4$ & Dodecanenitrile & $\mathrm{C}(\mathrm{CCCCCC}) \mathrm{CCCCC \# N}$ & 1.903 & 6 \\
\hline 643 & $\mathrm{p}$ & $112-42-5$ & n-Undecyl Alcohol & $\mathrm{C}(\mathrm{CCCCCO}) \mathrm{CCCCC}$ & 1.955 & 6,12 \\
\hline 644 & $\mathrm{p}$ & $2016-57-1$ & n-Decylamine & $\mathrm{C}(\mathrm{CCCCC}) \mathrm{CCCCN}$ & 2.056 & 6 \\
\hline 645 & $\mathrm{p}$ & $22104-81-0$ & trans-2-Dodecen-1-ol & $\mathrm{C}(\mathrm{CCCCCC}) \mathrm{CC} \backslash \mathrm{C}=\mathrm{C} \backslash \mathrm{CO}$ & 2.092 & 6 \\
\hline 646 & $\mathrm{p}$ & $3674-13-3$ & Ethyl 2,3-dibromopropionate & $\mathrm{C}([\mathrm{C} @ @ \mathrm{H}](\mathrm{CBr}) \mathrm{Br})(\mathrm{OCC})=\mathrm{O}$ & 2.208 & 6 \\
\hline 647 & $\mathrm{n}$ & $75-05-8$ & Acetonitrile & $\mathrm{C}(\mathrm{C}) \# \mathrm{~N}$ & -2.277 & 6 \\
\hline 648 & $\mathrm{n}$ & $67-64-1$ & Acetone & $\mathrm{C}(\mathrm{C})(\mathrm{C})=\mathrm{O}$ & -2.204 & 6,12 \\
\hline 649 & $\mathrm{n}$ & $26171-83-5$ & 1,2-Butanediol & $\mathrm{C}([\mathrm{C} @ @ \mathrm{H}](\mathrm{CO}) \mathrm{O}) \mathrm{C}$ & -2.048 & 6,12 \\
\hline 650 & $\mathrm{n}$ & $598-55-0$ & Methyl carbamate & $\mathrm{C}(\mathrm{OC})(\mathrm{N})=\mathrm{O}$ & -1.975 & 6 \\
\hline 651 & $\mathrm{n}$ & $2935-44-6$ & 2,5-Hexanediol & $\mathrm{C}(\mathrm{C}[\mathrm{C} @ \mathrm{H}](\mathrm{C}) \mathrm{O})[\mathrm{C} @ @ \mathrm{H}](\mathrm{C}) \mathrm{O}$ & -1.960 & 6 \\
\hline 652 & $\mathrm{n}$ & $111-29-5$ & 1,5-Pentanediol & $\mathrm{C}(\mathrm{CCO}) \mathrm{CCO}$ & -1.934 & 6,12 \\
\hline 653 & $\mathrm{n}$ & $107-18-6$ & Allyl alcohol & $\mathrm{C}(\mathrm{CO})=\mathrm{C}$ & -1.918 & 6,12 \\
\hline 654 & $\mathrm{n}$ & $67-63-0$ & 2-Propanol & $\mathrm{C}(\mathrm{C})(\mathrm{C}) \mathrm{O}$ & -1.882 & 6,12 \\
\hline 655 & $\mathrm{n}$ & $110-61-2$ & Succinonitrile & $\mathrm{C}(\mathrm{CC} \# \mathrm{~N}) \mathrm{CHN}$ & -1.824 & 6 \\
\hline 656 & $\mathrm{n}$ & $75-65-0$ & t-Butyl Alcohol & $\mathrm{C}(\mathrm{C})(\mathrm{C})(\mathrm{C}) \mathrm{O}$ & -1.791 & 6,12 \\
\hline 657 & $\mathrm{n}$ & $2508-29-4$ & 5-Amino-1-pentanol & $\mathrm{C}(\mathrm{CCN}) \mathrm{CCO}$ & -1.765 & 6 \\
\hline 658 & $\mathrm{n}$ & $71-23-8$ & n-Propanol & $\mathrm{C}(\mathrm{CO}) \mathrm{C}$ & -1.746 & 6,12 \\
\hline 659 & $\mathrm{n}$ & $96-48-0$ & gamma-Butyrolactone & $\mathrm{O}=\mathrm{C} 1 \mathrm{CCCO} 1$ & -1.717 & 6 \\
\hline 660 & $\mathrm{n}$ & $627-27-0$ & 3-Buten-1-ol & $\mathrm{C}(\mathrm{C}=\mathrm{C}) \mathrm{CO}$ & -1.695 & 6 \\
\hline 661 & $\mathrm{n}$ & $4620-70-6$ & 2-(1,1-dimethylethyl)amino-ethanol & $\mathrm{N}(\mathrm{C}(\mathrm{C})(\mathrm{C}) \mathrm{C}) \mathrm{CCO}$ & -1.673 & 6,12 \\
\hline 662 & $\mathrm{n}$ & $513-42-8$ & methallyl alcohol & $\mathrm{C}(\mathrm{CO})(\mathrm{C})=\mathrm{C}$ & -1.663 & 6 \\
\hline 663 & $\mathrm{n}$ & $51-79-6$ & urethane & $\mathrm{O}(\mathrm{C}(\mathrm{N})=\mathrm{O}) \mathrm{CC}$ & -1.650 & 6 \\
\hline 664 & $\mathrm{n}$ & $544-13-8$ & Pentanedinitrile & $\mathrm{C}(\mathrm{CC} \# \mathrm{~N}) \mathrm{CC} \# \mathrm{~N}$ & -1.638 & 6 \\
\hline 665 & $\mathrm{n}$ & $5343-92-0$ & 1,2-Pentanediol & $\mathrm{C}([\mathrm{C} @ @ \mathrm{H}](\mathrm{CO}) \mathrm{O}) \mathrm{CC}$ & -1.627 & 6,12 \\
\hline 666 & $\mathrm{n}$ & $107-29-9$ & acetaldehyde oxime & $\mathrm{C}(=\mathrm{N} \backslash \mathrm{O}) \backslash \mathrm{C}$ & -1.610 & 6 \\
\hline 667 & $\mathrm{n}$ & $79-20-9$ & Methyl Acetate & $\mathrm{C}(\mathrm{OC})(\mathrm{C})=\mathrm{O}$ & -1.595 & 6 \\
\hline
\end{tabular}




\begin{tabular}{|c|c|c|c|c|c|c|}
\hline 668 & $\mathrm{n}$ & $142-30-3$ & 2,5-Dimethyl-3-hexyne-2,5-diol & $\mathrm{C}(\# \mathrm{CC}(\mathrm{C})(\mathrm{C}) \mathrm{O}) \mathrm{C}(\mathrm{C})(\mathrm{C}) \mathrm{O}$ & -1.585 & 6 \\
\hline 669 & $\mathrm{n}$ & $108-29-2$ & gamma-Valerolactone & $\mathrm{C}[\mathrm{C} @ \mathrm{H}] 1 \mathrm{OC}(\mathrm{CC} 1)=\mathrm{O}$ & -1.569 & 6 \\
\hline 670 & $\mathrm{n}$ & $111-69-3$ & Adiponitrile & $\mathrm{C}(\mathrm{CCC} \# \mathrm{~N}) \mathrm{CC} \# \mathrm{~N}$ & -1.538 & 6 \\
\hline 671 & $\mathrm{n}$ & $107-31-3$ & Methyl Formate & $\mathrm{C}(\mathrm{OC})=\mathrm{O}$ & -1.498 & 6,12 \\
\hline 672 & $\mathrm{n}$ & $629-11-8$ & 1,6-Hexanediol & $\mathrm{C}(\mathrm{CCCO}) \mathrm{CCO}$ & -1.495 & 6,12 \\
\hline 673 & $\mathrm{n}$ & $109-75-1$ & Allyl Cyanide & $\mathrm{C}(\mathrm{C}=\mathrm{C}) \mathrm{C} \# \mathrm{~N}$ & -1.479 & 6 \\
\hline 674 & $\mathrm{n}$ & $96-22-0$ & Diethyl Ketone & $\mathrm{C}(\mathrm{CC})(\mathrm{CC})=\mathrm{O}$ & -1.456 & 6,12 \\
\hline 675 & $\mathrm{n}$ & $75-97-8$ & Pinacolin & $\mathrm{C}(\mathrm{C}(\mathrm{C})=\mathrm{O})(\mathrm{C})(\mathrm{C}) \mathrm{C}$ & -1.442 & 6 \\
\hline 676 & $\mathrm{n}$ & $71-36-3$ & n-Butanol & $\mathrm{C}(\mathrm{CC}) \mathrm{CO}$ & -1.431 & 6,12 \\
\hline 677 & $\mathrm{n}$ & $107-07-3$ & ethylene chlorohydrin & $\mathrm{C}(\mathrm{CO}) \mathrm{Cl}$ & -1.417 & 6,12 \\
\hline 678 & $\mathrm{n}$ & $110-13-4$ & 2,5-Hexanedione & $\mathrm{C}(\mathrm{CC}(\mathrm{C})=\mathrm{O}) \mathrm{C}(\mathrm{C})=\mathrm{O}$ & -1.403 & 6 \\
\hline 679 & $\mathrm{n}$ & $627-30-5$ & 3-chloro-1-propanol & $\mathrm{C}(\mathrm{CCl}) \mathrm{CO}$ & -1.399 & 6,12 \\
\hline 680 & $\mathrm{n}$ & $111-76-2$ & 2-Butoxy ethanol & $\mathrm{C}(\mathrm{OCCO}) \mathrm{CCC}$ & -1.374 & 6 \\
\hline 681 & $\mathrm{n}$ & $156-54-7$ & Butyric acid, sodium salt & $\mathrm{C}(\mathrm{C}(=\mathrm{O}) \mathrm{O}) \mathrm{CC}$ & -1.372 & 6 \\
\hline 682 & $\mathrm{n}$ & $616-25-1$ & 1-Penten-3-ol & $\mathrm{C}([\mathrm{C} @ @ \mathrm{H}](\mathrm{C}=\mathrm{C}) \mathrm{O}) \mathrm{C}$ & -1.348 & 6 \\
\hline 683 & $\mathrm{n}$ & $630-18-2$ & 2,2-dimethyl-propanenitrile & $\mathrm{C}(\mathrm{C} \# \mathrm{~N})(\mathrm{C})(\mathrm{C}) \mathrm{C}$ & -1.326 & 6 \\
\hline 684 & $\mathrm{n}$ & $115-19-5$ & 3-Methyl butynol & $\mathrm{C}(\mathrm{C} \# \mathrm{C})(\mathrm{C})(\mathrm{C}) \mathrm{O}$ & -1.311 & $6,12,14$ \\
\hline 685 & $\mathrm{n}$ & $141-78-6$ & ethyl acetate & $\mathrm{O}(\mathrm{C}(\mathrm{C})=\mathrm{O}) \mathrm{CC}$ & -1.297 & 6,12 \\
\hline 686 & $\mathrm{n}$ & $108-59-8$ & Dimethyl Malonate & $\mathrm{C}(\mathrm{C}(\mathrm{OC})=\mathrm{O}) \mathrm{C}(\mathrm{OC})=\mathrm{O}$ & -1.287 & 6,12 \\
\hline 687 & $\mathrm{n}$ & $6920-22-5$ & DL-1,2-Hexanediol & $\mathrm{C}([\mathrm{C} @ @ \mathrm{H}](\mathrm{CO}) \mathrm{O}) \mathrm{CCC}$ & -1.267 & 6,12 \\
\hline 688 & $\mathrm{n}$ & $127-06-0$ & Acetoxime & $\mathrm{C}(=\mathrm{N} / \mathrm{O})(\backslash \mathrm{C}) \mathrm{C}$ & -1.249 & 6 \\
\hline 689 & $\mathrm{n}$ & $584-02-1$ & 3-Pentanol & $\mathrm{C}(\mathrm{CC})(\mathrm{CC}) \mathrm{O}$ & -1.244 & 6,12 \\
\hline 690 & $\mathrm{n}$ & $556-82-1$ & 3-methyl-2-buten-1-ol & $\mathrm{C}(=\mathrm{C}(\backslash \mathrm{C}) \mathrm{C}) \backslash \mathrm{CO}$ & -1.239 & 6 \\
\hline 691 & $\mathrm{n}$ & $108-94-1$ & Cyclohexanone & $\mathrm{O}=\mathrm{C} 1 \mathrm{CCCCC} 1$ & -1.233 & 6 \\
\hline 692 & $\mathrm{n}$ & $4253-91-2$ & Dipropyl sulfoxide & $\mathrm{S}(\mathrm{CCC})(\mathrm{CCC})=\mathrm{O}$ & -1.221 & 6 \\
\hline 693 & $\mathrm{n}$ & $108-10-1$ & methyl isobutyl ketone & $\mathrm{C}(\mathrm{C}(\mathrm{C}) \mathrm{C}) \mathrm{C}(\mathrm{C})=\mathrm{O}$ & -1.209 & 6,12 \\
\hline 694 & $\mathrm{n}$ & $1679-47-6$ & Dihydro-3-methyl-2(3H)-furanone & $\mathrm{C}[\mathrm{C} @ \mathrm{H}] 1 \mathrm{C}(=\mathrm{O}) \mathrm{OCC} 1$ & -1.190 & 6 \\
\hline 695 & $\mathrm{n}$ & $10229-10-4$ & 3-Pentyn-1-ol & $\mathrm{C}(\mathrm{CCO}) \# \mathrm{CC}$ & -1.175 & 6 \\
\hline 696 & $\mathrm{n}$ & $563-80-4$ & Methyl Isopropyl Ketone & $\mathrm{C}(\mathrm{C}(\mathrm{C})=\mathrm{O})(\mathrm{C}) \mathrm{C}$ & -1.169 & 6 \\
\hline 697 & $\mathrm{n}$ & $627-09-8$ & Propargyl acetate & $\mathrm{O}(\mathrm{C}(\mathrm{C})=\mathrm{O}) \mathrm{CC} \# \mathrm{C}$ & -1.166 & 6 \\
\hline 698 & $\mathrm{n}$ & $109-49-9$ & 5-Hexen-2-one & $\mathrm{C}(\mathrm{C}(\mathrm{C})=\mathrm{O}) \mathrm{CC}=\mathrm{C}$ & -1.141 & 6 \\
\hline 699 & $\mathrm{n}$ & $107-19-7$ & Propargyl Alcohol & $\mathrm{C}(\mathrm{CO}) \# \mathrm{C}$ & -1.074 & 6,14 \\
\hline 700 & $\mathrm{n}$ & $106-65-0$ & Dimethyl Succinate & $\mathrm{C}(\mathrm{CCC}(\mathrm{OC})=\mathrm{O})(\mathrm{OC})=\mathrm{O}$ & -1.057 & 6,12 \\
\hline 701 & $\mathrm{n}$ & $123-51-3$ & Isoamyl Alcohol & $\mathrm{C}(\mathrm{C}(\mathrm{C}) \mathrm{C}) \mathrm{CO}$ & -1.036 & 6,12 \\
\hline 702 & $\mathrm{n}$ & $1002-28-4$ & 3-Hexyn-1-ol & $\mathrm{C}(\# \mathrm{CCC}) \mathrm{CCO}$ & -1.024 & 6 \\
\hline 703 & $\mathrm{n}$ & $110-59-8$ & n-Valeronitrile & $\mathrm{C}(\mathrm{CC} \# \mathrm{~N}) \mathrm{CC}$ & -1.007 & 6 \\
\hline 704 & $\mathrm{n}$ & $105-53-3$ & Diethyl malonate & $\mathrm{C}(\mathrm{CC}(\mathrm{OCC})=\mathrm{O})(\mathrm{OCC})=\mathrm{O}$ & -0.998 & 6,12 \\
\hline 705 & $\mathrm{n}$ & $598-38-9$ & 2,2-dichloroethanol & $\mathrm{C}(\mathrm{CO})(\mathrm{Cl}) \mathrm{Cl}$ & -0.990 & 6 \\
\hline 706 & $\mathrm{n}$ & $3538-65-6$ & Butryic acid hydrazide & $\mathrm{C}(\mathrm{CCC})(\mathrm{NN})=\mathrm{O}$ & -0.972 & 6 \\
\hline
\end{tabular}




\begin{tabular}{|c|c|c|c|c|c|c|}
\hline 707 & $\mathrm{n}$ & $4048-33-3$ & 6-Amino-1-hexanol & $\mathrm{C}(\mathrm{CCCO}) \mathrm{CCN}$ & -0.958 & 6,12 \\
\hline 708 & $\mathrm{n}$ & $16529-66-1$ & (E)-3-Pentenenitrile & $\mathrm{C}(\backslash \mathrm{CC} \# \mathrm{~N})=\mathrm{C} \backslash \mathrm{C}$ & -0.953 & 6 \\
\hline 709 & $\mathrm{n}$ & $110-15-6$ & Succinic acid & $\mathrm{C}(\mathrm{CC}(=\mathrm{O}) \mathrm{O}) \mathrm{C}(=\mathrm{O}) \mathrm{O}$ & -0.940 & 6,12 \\
\hline 710 & $\mathrm{n}$ & $627-18-9$ & 3-Bromo-1-propanol & $\mathrm{C}(\mathrm{CBr}) \mathrm{CO}$ & -0.933 & 6 \\
\hline 711 & $\mathrm{n}$ & $504-02-9$ & 1,3-Cyclohexanedione & $\mathrm{O}=\mathrm{C} 1 \mathrm{CC}(=\mathrm{O}) \mathrm{CCC} 1$ & -0.929 & 6 \\
\hline 712 & $\mathrm{n}$ & $2978-58-7$ & 1,1-Dimethylpropargylamine & $\mathrm{C}(\mathrm{C} \# \mathrm{C})(\mathrm{C})(\mathrm{C}) \mathrm{N}$ & -0.910 & 6,12 \\
\hline 713 & $\mathrm{n}$ & $75-64-9$ & 2-Amino-2-methylpropane & $\mathrm{C}(\mathrm{C})(\mathrm{C})(\mathrm{C}) \mathrm{N}$ & -0.897 & 6,12 \\
\hline 714 & $\mathrm{n}$ & $625-28-5$ & 3-methyl-butanenitrile & $\mathrm{C}(\mathrm{C}(\mathrm{C}) \mathrm{C}) \mathrm{C} \# \mathrm{~N}$ & -0.876 & 6 \\
\hline 715 & $\mathrm{n}$ & $75-84-3$ & Neopentyl Alcohol & $\mathrm{C}(\mathrm{CO})(\mathrm{C})(\mathrm{C}) \mathrm{C}$ & -0.870 & 6,12 \\
\hline 716 & $\mathrm{n}$ & $75-31-0$ & Isopropylamine & $\mathrm{C}(\mathrm{C})(\mathrm{C}) \mathrm{N}$ & -0.864 & 6,12 \\
\hline 717 & $\mathrm{n}$ & $108-05-4$ & Vinyl acetate & $\mathrm{O}(\mathrm{C}(\mathrm{C})=\mathrm{O}) \mathrm{C}=\mathrm{C}$ & -0.860 & 6 \\
\hline 718 & $\mathrm{n}$ & $540-51-2$ & Ethylene Bromohydrin & $\mathrm{C}(\mathrm{CO}) \mathrm{Br}$ & -0.846 & 6,12 \\
\hline 719 & $\mathrm{n}$ & $821-41-0$ & 5-Hexen-1-ol & $\mathrm{C}(\mathrm{CC}=\mathrm{C}) \mathrm{CCO}$ & -0.841 & 6,12 \\
\hline 720 & $\mathrm{n}$ & $2450-71-7$ & 2-Propyn-1-amine & $\mathrm{C}(\mathrm{CN}) \# \mathrm{C}$ & -0.826 & 6,12 \\
\hline 721 & $\mathrm{n}$ & $616-24-0$ & 3-Pentylamine & $\mathrm{C}(\mathrm{CC})(\mathrm{CC}) \mathrm{N}$ & -0.813 & 6,12 \\
\hline 722 & $\mathrm{n}$ & $928-96-1$ & cis-3-Hexen-1-ol & $\mathrm{C}(=\mathrm{C} / \mathrm{CC}) \backslash \mathrm{CCO}$ & -0.809 & $6,12,14$ \\
\hline 723 & $\mathrm{n}$ & $100-64-1$ & Cyclohexanone oxime & $\mathrm{N}(\backslash \mathrm{O})=\mathrm{C} 1 \backslash \mathrm{CCCCC} 1$ & -0.796 & 6 \\
\hline 724 & $\mathrm{n}$ & $542-54-1$ & Isocapronitrile & $\mathrm{C}(\mathrm{C}(\mathrm{C}) \mathrm{C}) \mathrm{CC} \# \mathrm{~N}$ & -0.786 & 6 \\
\hline 725 & $\mathrm{n}$ & $13401-56-4$ & 3-Chloro-2,2-dimethyl-1-propanol & $\mathrm{C}(\mathrm{CCl})(\mathrm{CO})(\mathrm{C}) \mathrm{C}$ & -0.782 & 6,12 \\
\hline 726 & $\mathrm{n}$ & $928-94-9$ & cis-2-Hexen-1-ol & $\mathrm{C}(\backslash \mathrm{CCC})=\mathrm{C} / \mathrm{CO}$ & -0.777 & 6,12 \\
\hline 727 & $\mathrm{n}$ & $108-93-0$ & Cyclohexanol & OC1CCCCC1 & -0.766 & 6 \\
\hline 728 & $\mathrm{n}$ & $36536-46-6$ & DL-3-butyrolactone & $\mathrm{C}[\mathrm{C} @ @ \mathrm{H}] 1 \mathrm{CC}(\mathrm{O} 1)=\mathrm{O}$ & -0.759 & 6 \\
\hline 729 & $\mathrm{n}$ & $928-92-7$ & 4-Hexen-1-ol & $\mathrm{C}(\backslash \mathrm{C}=\mathrm{C} \backslash \mathrm{C}) \mathrm{CCO}$ & -0.754 & 6,12 \\
\hline 730 & $\mathrm{n}$ & $108-64-5$ & ethyl isovalerate & $\mathrm{C}(\mathrm{C}(\mathrm{OCC})=\mathrm{O}) \mathrm{C}(\mathrm{C}) \mathrm{C}$ & -0.723 & 6,12 \\
\hline 731 & $\mathrm{n}$ & $598-74-3$ & 1,2-dimethylpropylamine & $\mathrm{C}([\mathrm{C} @ \mathrm{H}](\mathrm{C}) \mathrm{N})(\mathrm{C}) \mathrm{C}$ & -0.710 & 6,12 \\
\hline 732 & $\mathrm{n}$ & $107-10-8$ & n-Propyl Amine & $\mathrm{C}(\mathrm{CN}) \mathrm{C}$ & -0.708 & 6,12 \\
\hline 733 & $\mathrm{n}$ & $594-39-8$ & Tert-amylamine & $\mathrm{C}(\mathrm{CC})(\mathrm{C})(\mathrm{C}) \mathrm{N}$ & -0.698 & 6,12 \\
\hline 734 & $\mathrm{n}$ & $540-42-1$ & Isobutyl propionate & $\mathrm{C}(\mathrm{OCC}(\mathrm{C}) \mathrm{C})(\mathrm{CC})=\mathrm{O}$ & -0.694 & 6,12 \\
\hline 735 & $\mathrm{n}$ & $625-30-9$ & Sec-amylamine & $\mathrm{C}([\mathrm{C} @ \mathrm{H}](\mathrm{C}) \mathrm{N}) \mathrm{CC}$ & -0.685 & 6,12 \\
\hline 736 & $\mathrm{n}$ & $105-46-4$ & sec-Butyl Acetate & $\mathrm{O}([\mathrm{C} @ \mathrm{H}](\mathrm{CC}) \mathrm{C}) \mathrm{C}(\mathrm{C})=\mathrm{O}$ & -0.679 & 6 \\
\hline 737 & $\mathrm{n}$ & $627-00-9$ & Butanoic acid, 4-chloro- & $\mathrm{C}(\mathrm{C}(=\mathrm{O}) \mathrm{O}) \mathrm{CCCl}$ & -0.677 & 6,12 \\
\hline 738 & $\mathrm{n}$ & $33966-50-6$ & 2-Butanamine, (+-)- & $\mathrm{C}([\mathrm{C} @ \mathrm{H}](\mathrm{C}) \mathrm{N}) \mathrm{C}$ & -0.671 & 6,12 \\
\hline 739 & $\mathrm{n}$ & $4839-46-7$ & 3,3-Dimethylglutaric acid & $\mathrm{C}(\mathrm{C}(\mathrm{CC}(=\mathrm{O}) \mathrm{O})(\mathrm{C}) \mathrm{C}) \mathrm{C}(=\mathrm{O}) \mathrm{O}$ & -0.664 & 6,12 \\
\hline 740 & $\mathrm{n}$ & $1932-92-9$ & Propargyl propionate & $\mathrm{C}(\mathrm{OCC} \# \mathrm{C})(\mathrm{CC})=\mathrm{O}$ & -0.655 & 6 \\
\hline 741 & $\mathrm{n}$ & $110-12-3$ & Methyl Isoamyl Ketone & $\mathrm{C}(\mathrm{CC}(\mathrm{C})=\mathrm{O}) \mathrm{C}(\mathrm{C}) \mathrm{C}$ & -0.646 & 6,12 \\
\hline 742 & $\mathrm{n}$ & $110-94-1$ & Glutaric Acid & $\mathrm{C}(\mathrm{CC}(=\mathrm{O}) \mathrm{O}) \mathrm{CC}(=\mathrm{O}) \mathrm{O}$ & -0.639 & 6,12 \\
\hline 743 & $\mathrm{n}$ & $626-89-1$ & 4-methyl-1-pentanol & $\mathrm{C}(\mathrm{C}(\mathrm{C}) \mathrm{C}) \mathrm{CCO}$ & -0.637 & 6,12 \\
\hline 744 & $\mathrm{n}$ & $6280-87-1$ & 5-Chlorovaleronitrile & $\mathrm{C}(\mathrm{CCCl}) \mathrm{CC} \# \mathrm{~N}$ & -0.635 & 6 \\
\hline 745 & $\mathrm{n}$ & $19781-81-8$ & 4-Heptyn-2-ol & $\mathrm{C}(\mathrm{C} \# \mathrm{CCC})[\mathrm{C} @ \mathrm{H}](\mathrm{C}) \mathrm{O}$ & -0.616 & 6,12 \\
\hline
\end{tabular}




\begin{tabular}{|c|c|c|c|c|c|c|}
\hline 746 & $\mathrm{n}$ & $97-85-8$ & Isobutyl Isobutyrate & $\mathrm{C}(\mathrm{OCC}(\mathrm{C}) \mathrm{C})(\mathrm{C}(\mathrm{C}) \mathrm{C})=\mathrm{O}$ & -0.591 & 6 \\
\hline 747 & $\mathrm{n}$ & $111-16-0$ & Pimelic Acid & $\mathrm{C}(\mathrm{CC}(=\mathrm{O}) \mathrm{O}) \mathrm{CCCC}(=\mathrm{O}) \mathrm{O}$ & -0.585 & 6,12 \\
\hline 748 & $\mathrm{n}$ & $109-73-9$ & n-Butylamine & $\mathrm{C}(\mathrm{CC}) \mathrm{CN}$ & -0.574 & 6,12 \\
\hline 749 & $\mathrm{n}$ & $107-92-6$ & n-Butyric Acid & $\mathrm{C}(\mathrm{C}(=\mathrm{O}) \mathrm{O}) \mathrm{CC}$ & -0.572 & 6,12 \\
\hline 750 & $\mathrm{n}$ & $5557-88-0$ & 2-Penten-4-yn-1-ol & $\mathrm{C}(=\mathrm{C} \backslash \mathrm{CO}) \backslash \mathrm{C} \# \mathrm{C}$ & -0.555 & 6,12 \\
\hline 751 & $\mathrm{n}$ & $565-74-2$ & 2-Bromo-3-methylbutyric acid & $\mathrm{C}([\mathrm{C} @ \mathrm{H}](\mathrm{C}(=\mathrm{O}) \mathrm{O}) \mathrm{Br})(\mathrm{C}) \mathrm{C}$ & -0.549 & 6,12 \\
\hline 752 & $\mathrm{n}$ & $79-09-4$ & Propionic Acid & $\mathrm{C}(\mathrm{CC})(=\mathrm{O}) \mathrm{O}$ & -0.512 & 6 \\
\hline 753 & $\mathrm{n}$ & $609-08-5$ & Diethyl methylmalonate & $\mathrm{C}(\mathrm{C}(\mathrm{OCC})=\mathrm{O})(\mathrm{C}(\mathrm{OCC})=\mathrm{O}) \mathrm{C}$ & -0.511 & 6,12 \\
\hline 754 & $\mathrm{p}$ & $105-54-4$ & Ethyl butyrate & $\mathrm{C}(\mathrm{CCC})(\mathrm{OCC})=\mathrm{O}$ & -0.490 & 6,12 \\
\hline 755 & $\mathrm{p}$ & $110-43-0$ & Methyl n-amyl ketone & $\mathrm{C}(\mathrm{CCCC}) \mathrm{C}(\mathrm{C})=\mathrm{O}$ & -0.487 & 6,12 \\
\hline 756 & $\mathrm{p}$ & $96-13-9$ & 2,3-Dibromo-1-Propanol & $\mathrm{C}([\mathrm{C} @ \mathrm{H}](\mathrm{CO}) \mathrm{Br}) \mathrm{Br}$ & -0.486 & 6,12 \\
\hline 757 & $\mathrm{p}$ & $110-58-7$ & amylamine & $\mathrm{C}(\mathrm{CCN}) \mathrm{CC}$ & -0.485 & 6,12 \\
\hline 758 & $\mathrm{p}$ & $96-15-1$ & 2-Methylbutylamine & $\mathrm{C}([\mathrm{C} @ \mathrm{H}](\mathrm{CN}) \mathrm{C}) \mathrm{C}$ & -0.477 & 6,12 \\
\hline 759 & $\mathrm{p}$ & $928-95-0$ & trans-2-Hexen-1-ol & $\mathrm{C}(\backslash \mathrm{CCC})=\mathrm{C} \backslash \mathrm{CO}$ & -0.472 & 6,12 \\
\hline 760 & $\mathrm{p}$ & $115-20-8$ & Trichloroethanol & $\mathrm{C}(\mathrm{CO})(\mathrm{Cl})(\mathrm{Cl}) \mathrm{Cl}$ & -0.465 & 6 \\
\hline 761 & $\mathrm{p}$ & $40894-00-6$ & 3-Bromo-2,2-dimethyl-1-propanol & $\mathrm{C}(\mathrm{CBr})(\mathrm{CO})(\mathrm{C}) \mathrm{C}$ & -0.460 & 6 \\
\hline 762 & $\mathrm{p}$ & $78-84-2$ & Isobutyraldehyde & $\mathrm{C}(\mathrm{C}=\mathrm{O})(\mathrm{C}) \mathrm{C}$ & -0.433 & 6,12 \\
\hline 763 & $\mathrm{p}$ & $105-66-8$ & n-propyl butyrate & $\mathrm{C}(\mathrm{OCCC})(\mathrm{CCC})=\mathrm{O}$ & -0.414 & 6 \\
\hline 764 & $\mathrm{p}$ & $65337-13-5$ & DL-3-butyn-2-ol & $\mathrm{C} \# \mathrm{C}[\mathrm{CH}](\mathrm{O}) \mathrm{C}$ & -0.402 & $6,12,14$ \\
\hline 765 & $\mathrm{p}$ & $123-20-6$ & Vinyl butyrate & $\mathrm{C}(\mathrm{CCC})(\mathrm{OC}=\mathrm{C})=\mathrm{O}$ & -0.383 & 6 \\
\hline 766 & $\mathrm{p}$ & $7424-54-6$ & 3,5-heptadione & $\mathrm{C}(\mathrm{C}(\mathrm{CC})=\mathrm{O}) \mathrm{C}(=\mathrm{O}) \mathrm{CC}$ & -0.382 & 6 \\
\hline 767 & $\mathrm{p}$ & $104-50-7$ & gamma-Octanoic lactone & $\mathrm{C}(\mathrm{CCC})[\mathrm{C} @ \mathrm{H}] 1 \mathrm{OC}(\mathrm{CC} 1)=\mathrm{O}$ & -0.380 & 6 \\
\hline 768 & $\mathrm{p}$ & $40420-22-2$ & Diethyl 2,2-dimethyl-3-oxopimelate & $\begin{array}{l}\mathrm{C}(\mathrm{CCCC}(\mathrm{OCC})=\mathrm{O})(\mathrm{CC}(\mathrm{OCC})=\mathrm{O})= \\
\mathrm{O}\end{array}$ & -0.378 & 6 \\
\hline 769 & $\mathrm{p}$ & $628-73-9$ & n-Capronitrile & $\mathrm{C}(\mathrm{CCC}) \mathrm{CC} \# \mathrm{~N}$ & -0.376 & 6 \\
\hline 770 & $\mathrm{p}$ & $539-82-2$ & Ethyl n-Valerate & $\mathrm{C}(\mathrm{CCCC})(\mathrm{OCC})=\mathrm{O}$ & -0.358 & 6,12 \\
\hline 771 & $\mathrm{p}$ & $503-74-2$ & 3-Methylbutanoic acid & $\mathrm{C}(\mathrm{C}(\mathrm{C}) \mathrm{C}) \mathrm{C}(=\mathrm{O}) \mathrm{O}$ & -0.342 & 6,12 \\
\hline 772 & $\mathrm{p}$ & $107-94-8$ & 3-chloropropionic acid & $\mathrm{C}(\mathrm{C}(=\mathrm{O}) \mathrm{O}) \mathrm{CCl}$ & -0.332 & 6,12 \\
\hline 773 & $\mathrm{p}$ & $96-17-3$ & 2-Methylbutyraldehyde & $\mathrm{C}([\mathrm{C} @ \mathrm{H}](\mathrm{C}=\mathrm{O}) \mathrm{C}) \mathrm{C}$ & -0.311 & 6 \\
\hline 774 & $\mathrm{p}$ & $1119-46-6$ & 5-Chlorovaleric acid & $\mathrm{C}(\mathrm{CCCCl}) \mathrm{C}(=\mathrm{O}) \mathrm{O}$ & -0.286 & 6,12 \\
\hline 775 & $\mathrm{p}$ & $13991-37-2$ & trans-2-Pentenoic acid & $\mathrm{C}(\backslash \mathrm{C}(=\mathrm{O}) \mathrm{O})=\mathrm{C} \backslash \mathrm{CC}$ & -0.277 & 6 \\
\hline 776 & $\mathrm{p}$ & $2009-83-8$ & Hexamethylene Chlorohydrin & $\mathrm{C}(\mathrm{CCCO}) \mathrm{CCCl}$ & -0.273 & 6,12 \\
\hline 777 & $\mathrm{p}$ & $123-54-6$ & 2,4-Pentanedione & $\mathrm{C}(\mathrm{C}(\mathrm{C})=\mathrm{O}) \mathrm{C}(\mathrm{C})=\mathrm{O}$ & -0.272 & 6 \\
\hline 778 & $\mathrm{p}$ & $109-52-4$ & Valeric Acid & $\mathrm{C}(\mathrm{C}(=\mathrm{O}) \mathrm{O}) \mathrm{CCC}$ & -0.267 & 6,12 \\
\hline 779 & $\mathrm{p}$ & $78-81-9$ & Isobutylamine & $\mathrm{C}(\mathrm{CN})(\mathrm{C}) \mathrm{C}$ & -0.262 & 6,12 \\
\hline 780 & $\mathrm{p}$ & $598-04-9$ & n-Butyl sulfone & $\mathrm{S}(\mathrm{CCCC})(\mathrm{CCCC})(=\mathrm{O})=\mathrm{O}$ & -0.256 & 6 \\
\hline 781 & $\mathrm{p}$ & 133-13-1 & diethyl ethylmalonate & $\mathrm{C}(\mathrm{C}(\mathrm{OCC})=\mathrm{O})(\mathrm{C}(\mathrm{OCC})=\mathrm{O}) \mathrm{CC}$ & -0.242 & 6,12 \\
\hline 782 & $\mathrm{p}$ & $105-43-1$ & DL-3-Methylvaleric acid & $\mathrm{C}([\mathrm{C} @ \mathrm{H}](\mathrm{CC}) \mathrm{C}) \mathrm{C}(=\mathrm{O}) \mathrm{O}$ & -0.233 & 6,12 \\
\hline 783 & $\mathrm{p}$ & $111-26-2$ & Hexylamine & $\mathrm{C}(\mathrm{CCC}) \mathrm{CCN}$ & -0.220 & 6,12 \\
\hline
\end{tabular}




\begin{tabular}{|c|c|c|c|c|c|c|}
\hline 784 & $\mathrm{p}$ & $142-62-1$ & n-Hexanoic Acid & $\mathrm{C}(\mathrm{CCCC}) \mathrm{C}(=\mathrm{O}) \mathrm{O}$ & -0.208 & 6,12 \\
\hline 785 & $\mathrm{p}$ & $1002-36-4$ & 2-Heptyn-1-ol & $\mathrm{C}(\mathrm{C} \# \mathrm{CCO}) \mathrm{CCC}$ & -0.190 & 6 \\
\hline 786 & $\mathrm{p}$ & $66-25-1$ & hexaldehyde & $\mathrm{C}(\mathrm{CCC}) \mathrm{CC}=\mathrm{O}$ & -0.173 & 6,12 \\
\hline 787 & $\mathrm{p}$ & $88-09-5$ & 2-Ethylbutyric acid & $\mathrm{C}(\mathrm{C}(=\mathrm{O}) \mathrm{O})(\mathrm{CC}) \mathrm{CC}$ & -0.152 & 6,12 \\
\hline 788 & $\mathrm{p}$ & $20395-28-2$ & 5-Chloroamyl acetate & $\mathrm{C}(\mathrm{CCCCCl}) \mathrm{OC}(\mathrm{C})=\mathrm{O}$ & -0.131 & 6 \\
\hline 789 & $\mathrm{p}$ & $141-28-6$ & Diethyl Adipate & $\mathrm{C}(\mathrm{CCCCC}(\mathrm{OCC})=\mathrm{O})(\mathrm{OCC})=\mathrm{O}$ & -0.127 & 6,12 \\
\hline 790 & $\mathrm{p}$ & $111-14-8$ & Enanthoic Acid & $\mathrm{C}(\mathrm{CC}(=\mathrm{O}) \mathrm{O}) \mathrm{CCCC}$ & -0.113 & 6,12 \\
\hline 791 & $\mathrm{p}$ & $5454-83-1$ & Methyl 5-bromovalerate & $\mathrm{C}(\mathrm{CCCCBr})(\mathrm{OC})=\mathrm{O}$ & -0.080 & 6 \\
\hline 792 & $\mathrm{p}$ & $705-86-2$ & delta-decalactone & $\mathrm{C}(\mathrm{CCCC})[\mathrm{C} @ \mathrm{H}] 1 \mathrm{OC}(=\mathrm{O}) \mathrm{CCC} 1$ & -0.077 & 6 \\
\hline 793 & $\mathrm{p}$ & $5663-96-7$ & 2-Octynoic acid & $\mathrm{C}(\mathrm{CCCCC}) \# \mathrm{CC}(=\mathrm{O}) \mathrm{O}$ & -0.057 & 6 \\
\hline 794 & $\mathrm{p}$ & $97-96-1$ & ethyl butyraldehyde & $\mathrm{C}(\mathrm{CC})(\mathrm{CC}) \mathrm{C}=\mathrm{O}$ & -0.054 & 6,12 \\
\hline 795 & $\mathrm{p}$ & $624-54-4$ & Pentyl propionate & $\mathrm{C}(\mathrm{OCCCCC})(\mathrm{CC})=\mathrm{O}$ & -0.043 & 6,12 \\
\hline 796 & $\mathrm{p}$ & $584-93-0$ & 2-Bromovaleric acid & $\mathrm{C}([\mathrm{C} @ \mathrm{H}](\mathrm{C}(=\mathrm{O}) \mathrm{O}) \mathrm{Br}) \mathrm{CC}$ & -0.042 & 6,12 \\
\hline 797 & $\mathrm{p}$ & $2969-81-5$ & Ethyl 4-bromobutyrate & $\mathrm{C}(\mathrm{CCCBr})(\mathrm{OCC})=\mathrm{O}$ & -0.029 & 6 \\
\hline 798 & $\mathrm{p}$ & $110-62-3$ & n-Valeraldehyde & $\mathrm{C}(\mathrm{CC}=\mathrm{O}) \mathrm{CC}$ & -0.022 & 6,12 \\
\hline 799 & $\mathrm{p}$ & $1569-69-3$ & cyclohexylmercaptan & SC1CCCCC1 & -0.004 & 6 \\
\hline 800 & $\mathrm{p}$ & $111-71-7$ & n-Heptyl Aldehyde & $\mathrm{C}(\mathrm{CCC}=\mathrm{O}) \mathrm{CCC}$ & -0.002 & 6,12 \\
\hline 801 & $\mathrm{p}$ & $4286-55-9$ & 6-Bromo-1-hexanol & $\mathrm{C}(\mathrm{CCCO}) \mathrm{CCBr}$ & 0.007 & 6,12 \\
\hline 802 & $\mathrm{p}$ & $104-61-0$ & gamma-Nonanolactone & $\mathrm{C}(\mathrm{CCCC})[\mathrm{C} @ \mathrm{H}] 1 \mathrm{OC}(\mathrm{CC} 1)=\mathrm{O}$ & 0.014 & 6 \\
\hline 803 & $\mathrm{p}$ & $609-12-1$ & Ethyl 2-bromo-3-methylbutyrate & $\mathrm{C}([\mathrm{C} @ @ \mathrm{H}](\mathrm{C}(\mathrm{C}) \mathrm{C}) \mathrm{Br})(\mathrm{OCC})=\mathrm{O}$ & 0.021 & 6 \\
\hline 804 & $\mathrm{p}$ & $4224-70-8$ & 6-Bromohexanoic acid & $\mathrm{C}(\mathrm{CC}(=\mathrm{O}) \mathrm{O}) \mathrm{CCCBr}$ & 0.031 & 6 \\
\hline 805 & $\mathrm{p}$ & $33467-76-4$ & trans-2-Hepten-1-ol & $\mathrm{C}(\backslash \mathrm{C}=\mathrm{C} \backslash \mathrm{CO}) \mathrm{CCC}$ & 0.045 & 6 \\
\hline 806 & $\mathrm{p}$ & $502-56-7$ & 5-Nonanone & $\mathrm{C}(\mathrm{CCCC})(\mathrm{CCCC})=\mathrm{O}$ & 0.074 & 6 \\
\hline 807 & $\mathrm{p}$ & $124-07-2$ & Octanoic Acid & $\mathrm{C}(\mathrm{CCCCC}) \mathrm{CC}(=\mathrm{O}) \mathrm{O}$ & 0.081 & 6,12 \\
\hline 808 & $\mathrm{p}$ & $111-70-6$ & n-Heptyl Alcohol & $\mathrm{C}(\mathrm{CCCO}) \mathrm{CCC}$ & 0.105 & 6 \\
\hline 809 & $\mathrm{p}$ & $80-58-0$ & alpha-Bromobutyric acid & $\mathrm{C}([\mathrm{C} @ \mathrm{H}](\mathrm{CC}) \mathrm{Br})(=\mathrm{O}) \mathrm{O}$ & 0.122 & 6,12 \\
\hline 810 & $\mathrm{p}$ & $14090-87-0$ & 2,4-Octanedione & $\mathrm{C}(\mathrm{C}(\mathrm{CCCC})=\mathrm{O}) \mathrm{C}(\mathrm{C})=\mathrm{O}$ & 0.130 & 6 \\
\hline 811 & $\mathrm{p}$ & $58175-57-8$ & 2-Propyl-1-pentanol & $\mathrm{C}(\mathrm{CCC})(\mathrm{CCC}) \mathrm{CO}$ & 0.134 & 6 \\
\hline 812 & $\mathrm{p}$ & $123-05-7$ & 2-Ethylhexyl Aldehyde & $\mathrm{C}([\mathrm{C} @ \mathrm{H}](\mathrm{CC}) \mathrm{C}=\mathrm{O}) \mathrm{CCC}$ & 0.161 & 6,12 \\
\hline 813 & $\mathrm{p}$ & $104-76-7$ & 2-Ethyl-1-hexanol & $\mathrm{C}([\mathrm{C} @ @ \mathrm{H}](\mathrm{CC}) \mathrm{CO}) \mathrm{CCC}$ & 0.167 & 6 \\
\hline 814 & $\mathrm{p}$ & $20739-58-6$ & 2-Octyn-1-ol & $\mathrm{C}(\mathrm{CCCC}) \mathrm{C} \# \mathrm{CCO}$ & 0.194 & 6,12 \\
\hline 815 & $\mathrm{p}$ & $111-68-2$ & n-Heptylamine & $\mathrm{C}(\mathrm{CCCN}) \mathrm{CCC}$ & 0.211 & 6 \\
\hline 816 & $\mathrm{p}$ & $14660-52-7$ & Ethyl 5-bromovalerate & $\mathrm{C}(\mathrm{C}(\mathrm{OCC})=\mathrm{O}) \mathrm{CCCBr}$ & 0.218 & 6 \\
\hline 817 & $\mathrm{p}$ & $4091-39-8$ & 3-Chloro-2-butanone & $\mathrm{C}([\mathrm{C} @ @ \mathrm{H}](\mathrm{C}) \mathrm{Cl})(\mathrm{C})=\mathrm{O}$ & 0.269 & 6 \\
\hline 818 & $\mathrm{p}$ & $1732-09-8$ & Dimethyl suberate & $\mathrm{C}(\mathrm{CCCCCCC}(\mathrm{OC})=\mathrm{O})(\mathrm{OC})=\mathrm{O}$ & 0.296 & 6,12 \\
\hline 819 & $\mathrm{p}$ & $31333-13-8$ & 3-Nonyn-1-ol & $\mathrm{C}(\mathrm{C} \# \mathrm{CCCO}) \mathrm{CCCC}$ & 0.340 & 6,12 \\
\hline 820 & $\mathrm{p}$ & $111-86-4$ & 1-Aminooctane & $\mathrm{C}(\mathrm{CCCC}) \mathrm{CCCN}$ & 0.351 & 6 \\
\hline 821 & $\mathrm{p}$ & $597-97-7$ & tert-Amyl isothiocyanate & $\mathrm{C}(\mathrm{N}=\mathrm{C}=\mathrm{S})(\mathrm{CC})(\mathrm{C}) \mathrm{C}$ & 0.353 & 6 \\
\hline 822 & $\mathrm{p}$ & $4753-59-7$ & 1-Butanol, 4-bromo-, acetate & $\mathrm{C}(\mathrm{OC}(\mathrm{C})=\mathrm{O}) \mathrm{CCCBr}$ & 0.392 & 6 \\
\hline
\end{tabular}




\begin{tabular}{|c|c|c|c|c|c|c|}
\hline 823 & $\mathrm{p}$ & $2050-20-6$ & Diethyl pimelate & $\mathrm{C}(\mathrm{CCCCCC}(\mathrm{OCC})=\mathrm{O})(\mathrm{OCC})=\mathrm{O}$ & 0.407 & 6,12 \\
\hline 824 & $\mathrm{p}$ & $124-13-0$ & n-Octyl Aldehyde & $\mathrm{C}(\mathrm{CCCC}) \mathrm{CCC}=\mathrm{O}$ & 0.483 & 6 \\
\hline 825 & $\mathrm{p}$ & $706-14-9$ & gamma-Decalactone & $\mathrm{C}(\mathrm{CCCCC})[\mathrm{C} @ \mathrm{H}] 1 \mathrm{OC}(\mathrm{CC} 1)=\mathrm{O}$ & 0.493 & 6 \\
\hline 826 & $\mathrm{p}$ & $334-48-5$ & n-Decanoic Acid & $\mathrm{C}(\mathrm{CCCCCC}) \mathrm{CCC}(=\mathrm{O}) \mathrm{O}$ & 0.506 & 6,12 \\
\hline 827 & $\mathrm{p}$ & $111-11-5$ & methyl octanoate & $\mathrm{C}(\mathrm{CC}(\mathrm{OC})=\mathrm{O}) \mathrm{CCCCC}$ & 0.536 & 6,12 \\
\hline 828 & $\mathrm{p}$ & $693-54-9$ & 2-decanone & $\mathrm{C}(\mathrm{CCCCC}) \mathrm{CCC}(\mathrm{C})=\mathrm{O}$ & 0.582 & 6,12 \\
\hline 829 & $\mathrm{p}$ & $25542-62-5$ & Ethyl 6-bromohexanoate & $\mathrm{C}(\mathrm{CCCCCBr})(\mathrm{OCC})=\mathrm{O}$ & 0.594 & 6 \\
\hline 830 & $\mathrm{p}$ & $61996-79-0$ & 5-Methyl-1-hexyn-3-ol & $\mathrm{C}([\mathrm{C} @ \mathrm{H}](\mathrm{C} \# \mathrm{C}) \mathrm{O}) \mathrm{C}(\mathrm{C}) \mathrm{C}$ & 0.617 & 6 \\
\hline 831 & $\mathrm{p}$ & $625-22-9$ & Di-n-butyl sulfate & $\mathrm{O}(\mathrm{S}(\mathrm{OCCCC})(=\mathrm{O})=\mathrm{O}) \mathrm{CCCC}$ & 0.621 & 6 \\
\hline 832 & $\mathrm{p}$ & $19481-82-4$ & Propanenitrile, 2-bromo- & $\mathrm{C}([\mathrm{C} @ \mathrm{H}](\mathrm{Br}) \mathrm{C}) \# \mathrm{~N}$ & 0.626 & 6 \\
\hline 833 & $\mathrm{p}$ & $928-80-3$ & 3-Decanone & $\mathrm{C}(\mathrm{CC}(\mathrm{CC})=\mathrm{O}) \mathrm{CCCCC}$ & 0.627 & 6,12 \\
\hline 834 & $\mathrm{p}$ & $513-88-2$ & 1,1-dichloroacetone & $\mathrm{C}(\mathrm{C}(\mathrm{Cl}) \mathrm{Cl})(\mathrm{C})=\mathrm{O}$ & 0.641 & 6 \\
\hline 835 & $\mathrm{p}$ & $105-31-7$ & 1-Hexyn-3-ol & $\mathrm{C}([\mathrm{C} @ @ \mathrm{H}](\mathrm{C} \# \mathrm{C}) \mathrm{O}) \mathrm{CC}$ & 0.657 & 6,12 \\
\hline 836 & $\mathrm{p}$ & $615-83-8$ & DL-Ethyl 2-bromovalerate & $\mathrm{C}([\mathrm{C} @ \mathrm{H}](\mathrm{CCC}) \mathrm{Br})(\mathrm{OCC})=\mathrm{O}$ & 0.699 & 6 \\
\hline 837 & $\mathrm{p}$ & $1846-70-4$ & 2-Nonynoic acid & $\mathrm{C}(\mathrm{CCCCC}) \mathrm{C} \# \mathrm{CC}(=\mathrm{O}) \mathrm{O}$ & 0.714 & 6 \\
\hline 838 & $\mathrm{p}$ & $1119-86-4$ & 1,2-Decanediol & $\mathrm{C}(\mathrm{CC}[\mathrm{C} @ @ \mathrm{H}](\mathrm{CO}) \mathrm{O}) \mathrm{CCCCC}$ & 0.764 & 6,12 \\
\hline 839 & $\mathrm{p}$ & $22104-79-6$ & cis-2-Nonen-1-ol & $\mathrm{C}(\mathrm{C} \backslash \mathrm{C}=\mathrm{C} \backslash \mathrm{CO}) \mathrm{CCCC}$ & 0.770 & 6 \\
\hline 840 & $\mathrm{p}$ & $124-19-6$ & n-Nonyl Aldehyde & $\mathrm{C}(\mathrm{CCCC}=\mathrm{O}) \mathrm{CCCC}$ & 0.808 & 6 \\
\hline 841 & $\mathrm{p}$ & $2051-31-2$ & 4-Decanol & $\mathrm{C}([\mathrm{C} @ \mathrm{H}](\mathrm{CCC}) \mathrm{O}) \mathrm{CCCCC}$ & 0.850 & 6,12 \\
\hline 842 & $\mathrm{p}$ & $615-96-3$ & Hexanoic acid, 2-bromo-, ethyl ester & $\mathrm{C}([\mathrm{C} @ \mathrm{H}](\mathrm{CCCC}) \mathrm{Br})(\mathrm{OCC})=\mathrm{O}$ & 0.860 & 6 \\
\hline 843 & $\mathrm{p}$ & $111-25-1$ & n-Hexyl Bromide & $\mathrm{C}(\mathrm{CCBr}) \mathrm{CCC}$ & 0.937 & 6 \\
\hline 844 & $\mathrm{p}$ & $3018-12-0$ & Dichloroacetonitrile & $\mathrm{C}(\mathrm{C} \# \mathrm{~N})(\mathrm{Cl}) \mathrm{Cl}$ & 0.973 & 6 \\
\hline 845 & $\mathrm{p}$ & $1639-09-4$ & heptylmercaptan & $\mathrm{C}(\mathrm{CCCS}) \mathrm{CCC}$ & 1.020 & 6,12 \\
\hline 846 & $\mathrm{p}$ & $544-40-1$ & Butyl sulfide & $\mathrm{S}(\mathrm{CCCC}) \mathrm{CCCC}$ & 1.040 & 6 \\
\hline 847 & $\mathrm{p}$ & $50816-19-8$ & 8-Bromo-1-octanol & $\mathrm{C}(\mathrm{CCCCO}) \mathrm{CCCBr}$ & 1.042 & 6,12 \\
\hline 848 & $\mathrm{p}$ & 94-04-2 & Vinyl 2-ethylhexanoate & $\mathrm{C}([\mathrm{C} @ @ \mathrm{H}](\mathrm{CCCC}) \mathrm{CC})(\mathrm{OC}=\mathrm{C})=\mathrm{O}$ & 1.046 & 6 \\
\hline 849 & $\mathrm{p}$ & $535-11-5$ & 2-bromopropanoic acid ethyl ester & $\mathrm{C}(\mathrm{OCC})([\mathrm{C} @ \mathrm{H}](\mathrm{Br}) \mathrm{C})=\mathrm{O}$ & 1.057 & 6 \\
\hline 850 & $\mathrm{p}$ & $22104-80-9$ & trans-2-Decen-1-ol & $\mathrm{C}(\mathrm{CCCCC}) \mathrm{C} \backslash \mathrm{C}=\mathrm{C} \backslash \mathrm{CO}$ & 1.128 & 6 \\
\hline 851 & $\mathrm{p}$ & $1191-62-4$ & 1,8-Octanedithiol & $\mathrm{C}(\mathrm{CCCCS}) \mathrm{CCCS}$ & 1.191 & 6 \\
\hline 852 & $\mathrm{p}$ & $112-31-2$ & n-Decylaldehyde & $\mathrm{C}(\mathrm{CCCCC}) \mathrm{CCCC}=\mathrm{O}$ & 1.282 & 6 \\
\hline 853 & $\mathrm{p}$ & $112-30-1$ & 1-Decanol & $\mathrm{C}(\mathrm{CCCCC}) \mathrm{CCCCO}$ & 1.335 & 6,12 \\
\hline 854 & $\mathrm{p}$ & $110-42-9$ & methyl decanoate & $\mathrm{C}(\mathrm{CCCCCCCC}) \mathrm{C}(\mathrm{OC})=\mathrm{O}$ & 1.378 & 6,12 \\
\hline 855 & $\mathrm{p}$ & $609-11-0$ & Ethyl 2,3-dibromobutyrate & $\begin{array}{l}\mathrm{C}([\mathrm{C} @ @ \mathrm{H}]([\mathrm{C} @ @ \mathrm{H}](\mathrm{Br}) \mathrm{C}) \mathrm{Br})(\mathrm{O} \\
\mathrm{CC})=\mathrm{O}\end{array}$ & 1.438 & 6 \\
\hline 856 & $\mathrm{p}$ & $629-04-9$ & 1-Bromoheptane & $\mathrm{C}(\mathrm{CCCC}) \mathrm{CCBr}$ & 1.487 & 6 \\
\hline 857 & $\mathrm{p}$ & $112-12-9$ & 2-Undecanone & $\mathrm{C}(\mathrm{CCCCCC}) \mathrm{CCC}(\mathrm{C})=\mathrm{O}$ & 1.535 & 6,12 \\
\hline 858 & $\mathrm{p}$ & $542-85-8$ & Ethylisothiocyanate & $\mathrm{N}(\mathrm{CC})=\mathrm{C}=\mathrm{S}$ & 1.627 & 6 \\
\hline 859 & $\mathrm{p}$ & $1472-87-3$ & Tridecanedioic acid, dimethyl ester & $\begin{array}{l}\mathrm{C}(\mathrm{CCCCCCCCCCCC}(\mathrm{OC})=\mathrm{O})(\mathrm{OC}) \\
=\mathrm{O}\end{array}$ & 1.654 & 6,12 \\
\hline
\end{tabular}




\begin{tabular}{|c|c|c|c|c|c|c|}
\hline 860 & $\mathrm{p}$ & $6175-49-1$ & 2-dodecanone & $\mathrm{C}(\mathrm{CCCCCC}) \mathrm{CCCC}(\mathrm{C})=\mathrm{O}$ & 1.670 & 6,12 \\
\hline 861 & $\mathrm{p}$ & $628-30-8$ & n-Propyl isothiocyanate & $\mathrm{C}(\mathrm{N}=\mathrm{C}=\mathrm{S}) \mathrm{CC}$ & 1.693 & 6 \\
\hline 862 & $\mathrm{p}$ & $629-12-9$ & n-Amyl isothiocyanate & $\mathrm{C}(\mathrm{CN}=\mathrm{C}=\mathrm{S}) \mathrm{CCC}$ & 1.706 & 6 \\
\hline 863 & $\mathrm{p}$ & $4404-45-9$ & n-Hexyl isothiocyanate & $\mathrm{C}(\mathrm{CCCC}) \mathrm{CN}=\mathrm{C}=\mathrm{S}$ & 1.750 & 6 \\
\hline 864 & $\mathrm{p}$ & $111-83-1$ & 1-Bromooctane & $\mathrm{C}(\mathrm{CCCBr}) \mathrm{CCCC}$ & 1.870 & 6 \\
\hline 865 & $\mathrm{p}$ & $545-06-2$ & Trichloroacetonitrile & $\mathrm{C}(\mathrm{C} \# \mathrm{~N})(\mathrm{Cl})(\mathrm{Cl}) \mathrm{Cl}$ & 1.883 & 6 \\
\hline 866 & $\mathrm{p}$ & $821-06-7$ & (E)-1,4-dibromo-2-butene & $\mathrm{C}(=\mathrm{C} \backslash \mathrm{CBr}) \backslash \mathrm{CBr}$ & 1.925 & 6 \\
\hline 867 & $\mathrm{p}$ & $534-07-6$ & 1,3-dichloropropanone & $\mathrm{C}(\mathrm{CCl})(\mathrm{CCl})=\mathrm{O}$ & 2.046 & 6 \\
\hline 868 & $\mathrm{p}$ & $57-06-7$ & allyl isothiocyanate & $\mathrm{C}(\mathrm{N}=\mathrm{C}=\mathrm{S}) \mathrm{C}=\mathrm{C}$ & 2.061 & 6 \\
\hline 869 & $\mathrm{p}$ & $590-17-0$ & bromoacetonitrile & $\mathrm{C}(\mathrm{C} \# \mathrm{~N}) \mathrm{Br}$ & 2.229 & 6 \\
\hline 870 & $\mathrm{p}$ & $7307-55-3$ & n-Undecylamine & $\mathrm{C}(\mathrm{CCCCCN}) \mathrm{CCCCC}$ & 2.328 & 6 \\
\hline 871 & $\mathrm{p}$ & $5469-26-1$ & 1-Bromo-3,3-dimethyl-2-butanone & $\mathrm{C}(\mathrm{C}(\mathrm{C})(\mathrm{C}) \mathrm{C})(\mathrm{CBr})=\mathrm{O}$ & 2.377 & 6 \\
\hline 872 & $\mathrm{p}$ & $3252-43-5$ & Dibromoacetonitrile & $\mathrm{C}(\mathrm{CHN})(\mathrm{Br}) \mathrm{Br}$ & 2.398 & 6 \\
\hline 873 & $\mathrm{p}$ & $6802-75-1$ & Diethyl isopropylidenemalonate & $\begin{array}{l}\mathrm{C}(\backslash \mathrm{C}(\mathrm{OCC})=\mathrm{O})(\mathrm{C}(\mathrm{OCC})=\mathrm{O})=\mathrm{C}(/ \mathrm{C}) \\
\mathrm{C}\end{array}$ & -0.073 & 6 \\
\hline 874 & $\mathrm{p}$ & $2049-80-1$ & Diethyl allylmalonate & $\mathrm{C}(\mathrm{C}(\mathrm{OCC})=\mathrm{O})(\mathrm{C}(\mathrm{OCC})=\mathrm{O}) \mathrm{CC}=\mathrm{C}$ & -0.056 & 6 \\
\hline 875 & $\mathrm{p}$ & $590-92-1$ & 3-Bromopropionic acid & $\mathrm{C}(\mathrm{C}(=\mathrm{O}) \mathrm{O}) \mathrm{CBr}$ & -0.044 & \\
\hline 876 & $\mathrm{p}$ & $53723-19-6$ & 5-Nonyn-3-ol & $\mathrm{C}([\mathrm{C} @ @ \mathrm{H}](\mathrm{CC}) \mathrm{O}) \mathrm{C} \# \mathrm{CCCC}$ & -0.043 & 6 \\
\hline 877 & $\mathrm{p}$ & $32398-69-9$ & 4-Heptyn-3-ol & $\mathrm{C}([\mathrm{C} @ \mathrm{H}](\mathrm{CC}) \mathrm{O}) \# \mathrm{CCC}$ & -0.034 & 6,12 \\
\hline 878 & $\mathrm{p}$ & $565-68-4$ & 4-Methyl-1-pentyn-3-ol & $\mathrm{C}([\mathrm{C} @ \mathrm{H}](\mathrm{C} \# \mathrm{C}) \mathrm{O})(\mathrm{C}) \mathrm{C}$ & -0.027 & 6,12 \\
\hline 879 & $\mathrm{p}$ & $142-92-7$ & n-Hexyl Acetate & $\mathrm{C}(\mathrm{CCCCC}) \mathrm{OC}(\mathrm{C})=\mathrm{O}$ & -0.009 & 6,12 \\
\hline 880 & $\mathrm{p}$ & $111-47-7$ & Dipropyl sulfide & $\mathrm{S}(\mathrm{CCC}) \mathrm{CCC}$ & -0.003 & 6 \\
\hline 881 & $\mathrm{p}$ & $4128-31-8$ & $(+/-)-2-$ Octanol & $\mathrm{C}(\mathrm{C}[\mathrm{C} @ \mathrm{H}](\mathrm{C}) \mathrm{O}) \mathrm{CCCC}$ & 0.001 & 6,12 \\
\hline 882 & $\mathrm{p}$ & $141-06-0$ & n-Propyl pentanoate & $\mathrm{C}(\mathrm{CCCC})(\mathrm{OCCC})=\mathrm{O}$ & 0.009 & 6,12 \\
\hline 883 & $\mathrm{p}$ & $14916-80-4$ & 3-Octyn-1-ol & $\mathrm{C}(\mathrm{CCCC}) \# \mathrm{CCCO}$ & 0.017 & 6,12 \\
\hline 884 & $\mathrm{p}$ & $99-66-1$ & Valproic acid & $\mathrm{C}(\mathrm{CCC})(\mathrm{CCC}) \mathrm{C}(=\mathrm{O}) \mathrm{O}$ & 0.026 & 6,12 \\
\hline 885 & $\mathrm{p}$ & $20296-29-1$ & (+-)-3-octanol & $\mathrm{C}([\mathrm{C} @ @ \mathrm{H}](\mathrm{CC}) \mathrm{O}) \mathrm{CCCC}$ & 0.031 & 6,12 \\
\hline 886 & $\mathrm{p}$ & $123-66-0$ & Ethyl caproate & $\mathrm{C}(\mathrm{C}(\mathrm{OCC})=\mathrm{O}) \mathrm{CCCC}$ & 0.064 & 6,12 \\
\hline 887 & $\mathrm{p}$ & $149-57-5$ & 2-ethylhexanoic acid & $\mathrm{C}([\mathrm{C} @ \mathrm{H}](\mathrm{C}(=\mathrm{O}) \mathrm{O}) \mathrm{CC}) \mathrm{CCC}$ & 0.076 & 6,12 \\
\hline 888 & $\mathrm{p}$ & $623-98-3$ & Di-n-propyl sulfite & $\mathrm{S}(\mathrm{OCCC})(\mathrm{OCCC})=\mathrm{O}$ & 0.086 & 6 \\
\hline 889 & $\mathrm{p}$ & $106-73-0$ & Methyl heptanoate & $\mathrm{C}(\mathrm{CCCCCC})(\mathrm{OC})=\mathrm{O}$ & 0.104 & 6,12 \\
\hline 890 & $\mathrm{p}$ & $75-80-9$ & 2,2,2-tribromo-ethanol & $\mathrm{C}(\mathrm{CO})(\mathrm{Br})(\mathrm{Br}) \mathrm{Br}$ & 0.113 & 6 \\
\hline 891 & $\mathrm{p}$ & $539-74-2$ & Ethyl 3-bromopropionate & $\mathrm{C}(\mathrm{CCBr})(\mathrm{OCC})=\mathrm{O}$ & 0.126 & 6 \\
\hline 892 & $\mathrm{p}$ & $2163-48-6$ & Diethyl propylmalonate & $\mathrm{C}(\mathrm{C}(\mathrm{OCC})=\mathrm{O})(\mathrm{C}(\mathrm{OCC})=\mathrm{O}) \mathrm{CCC}$ & 0.134 & 6,12 \\
\hline 893 & $\mathrm{p}$ & $600-00-0$ & Ethyl 2-bromoisobutyrate & $\mathrm{C}(\mathrm{C}(\mathrm{Br})(\mathrm{C}) \mathrm{C})(\mathrm{OCC})=\mathrm{O}$ & 0.152 & 6 \\
\hline 894 & $\mathrm{p}$ & $628-63-7$ & Amyl acetate (mixed isomers) & $\mathrm{C}(\mathrm{OC}(\mathrm{C})=\mathrm{O}) \mathrm{CCCC}$ & 0.163 & 6,12 \\
\hline 895 & $\mathrm{p}$ & $590-01-2$ & n-Butyl Propionate & $\mathrm{C}(\mathrm{OCCCC})(\mathrm{CC})=\mathrm{O}$ & 0.170 & 6,12 \\
\hline 896 & $\mathrm{p}$ & $1871-67-6$ & E-2-Octenoic acid & $\mathrm{C}(\backslash \mathrm{CCCCC})=\mathrm{C} \backslash \mathrm{C}(=\mathrm{O}) \mathrm{O}$ & 0.209 & 6 \\
\hline 897 & $\mathrm{p}$ & $123-68-2$ & Allyl hexanoate & $\mathrm{C}(\mathrm{C}(\mathrm{OCC}=\mathrm{C})=\mathrm{O}) \mathrm{CCCC}$ & 0.213 & 6 \\
\hline
\end{tabular}




\begin{tabular}{|c|c|c|c|c|c|c|}
\hline 898 & $\mathrm{p}$ & $112-47-0$ & 1,10-Decanediol & $\mathrm{C}(\mathrm{CCCCCO}) \mathrm{CCCCO}$ & 0.224 & 6,12 \\
\hline 899 & $\mathrm{p}$ & $124-12-9$ & Octanenitrile & $\mathrm{C}(\mathrm{CCCC}) \mathrm{CCC \# N}$ & 0.283 & 6 \\
\hline 900 & $\mathrm{p}$ & $15848-22-3$ & 5-Bromoamyl acetate & $\mathrm{C}(\mathrm{CCCCBr}) \mathrm{OC}(\mathrm{C})=\mathrm{O}$ & 0.293 & 6 \\
\hline 901 & $\mathrm{p}$ & $142-61-0$ & Hexanoyl chloride & $\mathrm{C}(\mathrm{CCCC}) \mathrm{C}(\mathrm{Cl})=\mathrm{O}$ & 0.340 & 6 \\
\hline 902 & $\mathrm{p}$ & $78-69-3$ & 3,7-Dimethyl-3-octanol & $\mathrm{C}([\mathrm{C} @ @](\mathrm{CC})(\mathrm{C}) \mathrm{O}) \mathrm{CCC}(\mathrm{C}) \mathrm{C}$ & 0.340 & 6,12 \\
\hline 903 & $\mathrm{p}$ & $18409-17-1$ & (E)-2-octenol & $\mathrm{C}(\mathrm{CCCC}) \backslash \mathrm{C}=\mathrm{C} \backslash \mathrm{CO}$ & 0.365 & 6,12 \\
\hline 904 & $\mathrm{p}$ & $60657-70-7$ & 2-Methyl-5-octyn-4-ol & $\mathrm{C}([\mathrm{C} @ @ \mathrm{H}](\mathrm{C} \# \mathrm{CCC}) \mathrm{O}) \mathrm{C}(\mathrm{C}) \mathrm{C}$ & 0.401 & 6 \\
\hline 905 & $\mathrm{p}$ & $111-64-8$ & n-Capryloyl Chloride & $\mathrm{C}(\mathrm{CCCCC}) \mathrm{CC}(\mathrm{Cl})=\mathrm{O}$ & 0.415 & 6 \\
\hline 906 & $\mathrm{p}$ & $2528-61-2$ & Heptanoyl chloride & $\mathrm{C}(\mathrm{CC}(\mathrm{Cl})=\mathrm{O}) \mathrm{CCCC}$ & 0.459 & 6 \\
\hline 907 & $\mathrm{p}$ & $110-53-2$ & n-Amyl Bromide & $\mathrm{C}(\mathrm{CCC}) \mathrm{CBr}$ & 0.484 & 6 \\
\hline 908 & $\mathrm{p}$ & $109-50-2$ & 3-Hexyn-2-ol & $\mathrm{C}([\mathrm{C} @ \mathrm{H}](\mathrm{C}) \mathrm{O}) \# \mathrm{CCC}$ & 0.511 & 6 \\
\hline 909 & $\mathrm{p}$ & $141-03-7$ & Butanedioic acid, dibutyl ester & $\mathrm{C}(\mathrm{CCC}(\mathrm{OCCCC})=\mathrm{O})(\mathrm{OCCCC})=\mathrm{O}$ & 0.512 & 6,12 \\
\hline 910 & $\mathrm{p}$ & $109-21-7$ & Butyl butyrate & $\mathrm{O}(\mathrm{C}(\mathrm{CCC})=\mathrm{O}) \mathrm{CCCC}$ & 0.516 & 6,12 \\
\hline 911 & $\mathrm{p}$ & $133-08-4$ & Diethyl n-butylmalonate & $\mathrm{C}(\mathrm{C}(\mathrm{OCC})=\mathrm{O})(\mathrm{C}(\mathrm{OCC})=\mathrm{O}) \mathrm{CCCC}$ & 0.557 & 6,12 \\
\hline 912 & $\mathrm{p}$ & $111-87-5$ & Octyl alcohol & $\mathrm{C}(\mathrm{CCCC}) \mathrm{CCCO}$ & 0.583 & 6,12 \\
\hline 913 & $\mathrm{p}$ & $3760-11-0$ & 2-Nonenoic acid & $\mathrm{C}(\mathrm{CCCCC}) \backslash \mathrm{C}=\mathrm{C} \backslash \mathrm{C}(=\mathrm{O}) \mathrm{O}$ & 0.600 & 6 \\
\hline 914 & $\mathrm{p}$ & $628-99-9$ & 2-Nonanol & $\mathrm{C}(\mathrm{CCCCC}) \mathrm{C}[\mathrm{C} @ \mathrm{H}](\mathrm{C}) \mathrm{O}$ & 0.618 & 6,12 \\
\hline 915 & $\mathrm{p}$ & $2243-27-8$ & Nonanenitrile & $\mathrm{C}(\mathrm{CCCC} \# \mathrm{~N}) \mathrm{CCCC}$ & 0.625 & 6 \\
\hline 916 & $\mathrm{p}$ & $1191-43-1$ & 1,6-Hexanedithiol & $\mathrm{C}(\mathrm{CCCS}) \mathrm{CCS}$ & 0.626 & 6 \\
\hline 917 & $\mathrm{p}$ & $14064-10-9$ & chloropropanedioic acid diethyl ester & $\mathrm{C}(\mathrm{C}(\mathrm{OCC})=\mathrm{O})(\mathrm{C}(\mathrm{OCC})=\mathrm{O}) \mathrm{Cl}$ & 0.635 & 6 \\
\hline 918 & $\mathrm{p}$ & $5921-73-3$ & 2-Nonyn-1-ol & $\mathrm{C}(\mathrm{CC} \# \mathrm{CCO}) \mathrm{CCCC}$ & 0.649 & 6,12 \\
\hline 919 & $\mathrm{p}$ & $103-09-3$ & 2-Ethyl-1-hexyl acetate & $\mathrm{C}([\mathrm{C} @ @ \mathrm{H}](\mathrm{CCCC}) \mathrm{CC}) \mathrm{OC}(\mathrm{C})=\mathrm{O}$ & 1.057 & 11 \\
\hline 920 & $\mathrm{p}$ & $112-05-0$ & 1-Octanecarboxylic acid & $\mathrm{C}(\mathrm{CCCCC}) \mathrm{CCC}(=\mathrm{O}) \mathrm{O}$ & 0.3509 & 11 \\
\hline 921 & $\mathrm{p}$ & $112-17-4$ & 1-Decanol acetate & $\mathrm{C}(\mathrm{CCCCCCCC}) \mathrm{COC}(\mathrm{C})=\mathrm{O}$ & 1.8794 & 11 \\
\hline 922 & $\mathrm{p}$ & $112-53-8$ & 1-Dodecyl alcohol & $\mathrm{C}(\mathrm{CCCCCC}) \mathrm{CCCCCO}$ & 2.1612 & 11 \\
\hline 923 & $\mathrm{p}$ & $112-70-9$ & Tridecyl alcohol & $\mathrm{C}(\mathrm{CCCCCCO}) \mathrm{CCCCCC}$ & 2.4497 & 11 \\
\hline 924 & $\mathrm{p}$ & $123-96-6$ & 1-Methylheptyl alcohol & $\mathrm{C}(\mathrm{C}[\mathrm{C} @ \mathrm{H}](\mathrm{C}) \mathrm{O}) \mathrm{CCCC}$ & 0.0011 & 11 \\
\hline 925 & $\mathrm{p}$ & $462-18-0$ & Di-n-hexyl ketone & $\mathrm{C}(\mathrm{C}(\mathrm{CCCCCC})=\mathrm{O}) \mathrm{CCCCC}$ & 1.5214 & 11 \\
\hline 926 & $\mathrm{p}$ & $589-98-0$ & DL-3-Octanol & $\mathrm{C}([\mathrm{C} @ @ \mathrm{H}](\mathrm{CC}) \mathrm{O}) \mathrm{CCCC}$ & 0.0309 & 11 \\
\hline 927 & $\mathrm{p}$ & $616-05-7$ & 2-Bromohexanoic acid & $\mathrm{C}([\mathrm{C} @ \mathrm{H}](\mathrm{CCCC}) \mathrm{Br})(=\mathrm{O}) \mathrm{O}$ & 0.4547 & 11 \\
\hline 928 & $\mathrm{p}$ & $2028-63-9$ & 1-Butyn-3-ol & $\mathrm{C}([\mathrm{C} @ \mathrm{H}](\mathrm{C}) \mathrm{O}) \# \mathrm{C}$ & -0.4024 & 11 \\
\hline 929 & $\mathrm{p}$ & $2623-82-7$ & 2-Bromooctanoic acid & $\mathrm{C}([\mathrm{C} @ \mathrm{H}](\mathrm{C}(=\mathrm{O}) \mathrm{O}) \mathrm{Br}) \mathrm{CCCCC}$ & 0.4907 & 6,12 \\
\hline 930 & $\mathrm{p}$ & $5197-62-6$ & 8-Chloro-1-octanol & $\mathrm{C}(\mathrm{COCCO}) \mathrm{OCCCl}$ & 0.4878 & 12 \\
\hline 931 & $\mathrm{p}$ & $51887-25-3$ & 2-Tridecyn-1-ol & $\mathrm{C}(\mathrm{CCCC} \# \mathrm{CCO}) \mathrm{CCCCCC}$ & 2.3665 & 12 \\
\hline 932 & $\mathrm{p}$ & $360078-17-7$ & $\begin{array}{l}\text { 1-Heptyn-3-ol, 4-methyl-, (3S,4R)- } \\
\text { (9CI) }\end{array}$ & $\mathrm{C}([\mathrm{C} @ \mathrm{H}]([\mathrm{C} @ @ \mathrm{H}](\mathrm{C} \# \mathrm{C}) \mathrm{O}) \mathrm{C}) \mathrm{CC}$ & 0.7426 & 12 \\
\hline 933 & $\mathrm{n}$ & $78-92-2$ & 1-Methyl-1-propanol & $\mathrm{C}([\mathrm{C} @ \mathrm{H}](\mathrm{C}) \mathrm{O}) \mathrm{C}$ & -1.5420 & 12 \\
\hline 934 & $\mathrm{n}$ & $105-59-9$ & $N$-Methyldiethanol amine & $\mathrm{N}(\mathrm{CCO})(\mathrm{CCO}) \mathrm{C}$ & -1.8338 & 12 \\
\hline 935 & $\mathrm{n}$ & $107-88-0$ & $( \pm)$-1,3-Butanediol & $\mathrm{C}([\mathrm{C} @ \mathrm{H}](\mathrm{C}) \mathrm{O}) \mathrm{CO}$ & -2.3013 & 12 \\
\hline
\end{tabular}




\begin{tabular}{|c|c|c|c|c|c|c|}
\hline 936 & $\mathrm{n}$ & $111-42-2$ & Diethanolamine & $\mathrm{N}(\mathrm{CCO}) \mathrm{CCO}$ & -1.7941 & 12 \\
\hline 937 & $\mathrm{n}$ & $584-03-2$ & 1,2-Butylene glycol & $\mathrm{C}([\mathrm{C} @ @ \mathrm{H}](\mathrm{CO}) \mathrm{O}) \mathrm{C}$ & -2.0482 & 12 \\
\hline 938 & $\mathrm{n}$ & $616-29-5$ & 1,3-Diamino-2-hydroxy-propane & $\mathrm{C}(\mathrm{CN})(\mathrm{CN}) \mathrm{O}$ & -1.4275 & 12 \\
\hline 939 & $\mathrm{n}$ & $3724-65-0$ & Crotonic acid & $\mathrm{C}(\backslash \mathrm{C}(=\mathrm{O}) \mathrm{O})=\mathrm{C} \backslash \mathrm{C}$ & -0.5448 & 12 \\
\hline 940 & $\mathrm{n}$ & $24629-25-2$ & 2-Amino-3-methyl-1-pentanol & $\mathrm{C}([\mathrm{C} @ @ \mathrm{H}]([\mathrm{C} @ @ \mathrm{H}](\mathrm{CO}) \mathrm{N}) \mathrm{C}) \mathrm{C}$ & -0.6594 & 6,12 \\
\hline 941 & $\mathrm{n}$ & $5332-73-0$ & 3-Methoxypropylamine & $\mathrm{C}(\mathrm{COC}) \mathrm{CN}$ & -1.7725 & 12 \\
\hline 942 & $\mathrm{n}$ & $6032-29-7$ & 2-Pentanol & $\mathrm{C}([\mathrm{C} @ \mathrm{H}](\mathrm{C}) \mathrm{O}) \mathrm{CC}$ & -1.1596 & 6,12 \\
\hline 943 & $\mathrm{n}$ & $6126-50-7$ & 4-Hexen-1-ol & $\mathrm{C}(\backslash \mathrm{C}=\mathrm{C} \backslash \mathrm{C}) \mathrm{CCO}$ & -0.7540 & 12 \\
\hline 944 & $\mathrm{n}$ & $13325-10-5$ & 4-Amino-1-butanol & $\mathrm{C}(\mathrm{CCO}) \mathrm{CN}$ & -0.9752 & 12 \\
\hline 945 & $\mathrm{n}$ & $33966-50-6$ & $( \pm)$-sec-Butylamine & $\mathrm{C}([\mathrm{C} @ \mathrm{H}](\mathrm{C}) \mathrm{N}) \mathrm{C}$ & -0.6708 & 6,12 \\
\hline 946 & $\mathrm{n}$ & $16369-14-5$ & dl-2-Amino-1-pentanol & $\mathrm{C}([\mathrm{C} @ @ \mathrm{H}](\mathrm{CO}) \mathrm{N}) \mathrm{CC}$ & -0.6718 & 12 \\
\hline 947 & $\mathrm{n}$ & $16397-19-6$ & dl-2-Amino-1-hexanol & $\mathrm{C}([\mathrm{C} @ @ \mathrm{H}](\mathrm{CO}) \mathrm{N}) \mathrm{CCC}$ & -0.5848 & 12 \\
\hline 948 & $\mathrm{n}$ & $26734-09-8$ & 3-Amino-2,2-dimethyl-1-propanol & $\mathrm{C}(\mathrm{CN})(\mathrm{CO})(\mathrm{C}) \mathrm{C}$ & -0.9246 & 6,12 \\
\hline 949 & $\mathrm{n}$ & $40137-22-2$ & 3-(Methylamino)-1,2-propanediol & $\mathrm{C}([\mathrm{C} @ @ \mathrm{H}](\mathrm{CO}) \mathrm{O}) \mathrm{NC}$ & -1.5341 & 12 \\
\hline 950 & $\mathrm{n}$ & $63493-28-7$ & 1-Methylbutylamine & $\mathrm{C}([\mathrm{C} @ \mathrm{H}](\mathrm{C}) \mathrm{N}) \mathrm{CC}$ & -0.6846 & 6,12 \\
\hline 951 & $\mathrm{n}$ & $126-98-7$ & methacrylonitrile & $\mathrm{C}(\mathrm{C} \# \mathrm{~N})(\mathrm{C})=\mathrm{C}$ & -1.653 & 9 \\
\hline 952 & $\mathrm{n}$ & $2758-18-1$ & 3-methyl-2-cyclopenten-1-one & $\mathrm{CC} 1=\mathrm{CC}(\mathrm{CC} 1)=\mathrm{O}$ & -1.323 & 9 \\
\hline 953 & $\mathrm{n}$ & $80-62-6$ & methyl methacrylate & $\mathrm{C}(\mathrm{C}(\mathrm{C})=\mathrm{C})(\mathrm{OC})=\mathrm{O}$ & -1.218 & 9,16 \\
\hline 954 & $\mathrm{n}$ & $924-50-5$ & methyl-3,3-dimethylacrylate & $\mathrm{C}(\backslash \mathrm{C}=\mathrm{C}(\backslash \mathrm{C}) \mathrm{C})(\mathrm{OC})=\mathrm{O}$ & -1.061 & 9 \\
\hline 955 & $\mathrm{n}$ & $97-63-2$ & ethyl methacrylate & $\mathrm{C}(\mathrm{OCC})(\mathrm{C}(\mathrm{C})=\mathrm{C})=\mathrm{O}$ & -0.935 & 9 \\
\hline 956 & $\mathrm{n}$ & $623-43-8$ & methyl crotonate & $\mathrm{C}(\backslash \mathrm{C}=\mathrm{C} \backslash \mathrm{C})(\mathrm{OC})=\mathrm{O}$ & -0.918 & 9 \\
\hline 957 & $\mathrm{n}$ & $4655-34-9$ & isopropyl methacrylate & $\mathrm{C}(\mathrm{OC}(\mathrm{C}) \mathrm{C})(\mathrm{C}(\mathrm{C})=\mathrm{C})=\mathrm{O}$ & -0.881 & 9 \\
\hline 958 & $\mathrm{n}$ & $1120-73-6$ & 2-methyl-2-cyclopenten-1-one & $\mathrm{CC}=1 \mathrm{C}(=\mathrm{O}) \mathrm{CCC} 1$ & -0.826 & 9 \\
\hline 959 & $\mathrm{n}$ & $18060-77-0$ & crotonic acid isopropyl ester & $\mathrm{O}(\mathrm{C}(\backslash \mathrm{C}=\mathrm{C} \backslash \mathrm{C})=\mathrm{O}) \mathrm{C}(\mathrm{C}) \mathrm{C}$ & -0.782 & 9 \\
\hline 960 & $\mathrm{n}$ & $2370-63-0$ & 2-ethoxyethyl methacrylate & $\mathrm{C}(\mathrm{OCCOCC})(\mathrm{C}(\mathrm{C})=\mathrm{C})=\mathrm{O}$ & -0.779 & 9 \\
\hline 961 & $\mathrm{n}$ & $623-70-1$ & trans-ethyl crotonate & $\mathrm{C}(\backslash \mathrm{C}=\mathrm{C} \backslash \mathrm{C})(\mathrm{OCC})=\mathrm{O}$ & -0.765 & 9 \\
\hline 962 & $\mathrm{n}$ & $96-05-9$ & allyl methacrylate & $\mathrm{C}(\mathrm{C}(\mathrm{C})=\mathrm{C})(\mathrm{OCC}=\mathrm{C})=\mathrm{O}$ & -0.677 & 9 \\
\hline 963 & $\mathrm{n}$ & $2210-28-8$ & $n$-propyl methacrylate & $\mathrm{C}(\mathrm{C}(\mathrm{C})=\mathrm{C})(\mathrm{OCCC})=\mathrm{O}$ & -0.655 & 9 \\
\hline 964 & $\mathrm{n}$ & $141-79-7$ & 4-methyl-3-penten-2-one & $\mathrm{C}(=\mathrm{C}(\backslash \mathrm{C}) \mathrm{C}) \backslash \mathrm{C}(\mathrm{C})=\mathrm{O}$ & -0.644 & 9 \\
\hline 965 & $\mathrm{n}$ & $1663-39-4$ & tert-butyl acrylate & $\mathrm{O}(\mathrm{C}(\mathrm{C})(\mathrm{C}) \mathrm{C}) \mathrm{C}(\mathrm{C}=\mathrm{C})=\mathrm{O}$ & -0.64 & 9 \\
\hline 966 & $\mathrm{n}$ & $79218-15-8$ & crotonic acid tert-butyl ester & $\mathrm{C}(\mathrm{OC}(\mathrm{C})(\mathrm{C}) \mathrm{C})(\backslash \mathrm{C}=\mathrm{C} \backslash \mathrm{C})=\mathrm{O}$ & -0.639 & 9 \\
\hline 967 & $\mathrm{n}$ & $4245-37-8$ & vinyl methacrylate & $\mathrm{C}(\mathrm{OC}=\mathrm{C})(\mathrm{C}(\mathrm{C})=\mathrm{C})=\mathrm{O}$ & -0.619 & 9 \\
\hline 968 & $\mathrm{n}$ & $6622-76-0$ & methyl tiglate & $\mathrm{C}(\backslash \mathrm{C}(\mathrm{OC})=\mathrm{O})(=\mathrm{C} \backslash \mathrm{C}) \mathrm{C}$ & -0.617 & 9 \\
\hline 969 & $\mathrm{p}$ & $5837-78-5$ & tiglic acid ethyl ester & $\mathrm{C}(\backslash \mathrm{C}(=\mathrm{C} \backslash \mathrm{C}) \mathrm{C})(\mathrm{OCC})=\mathrm{O}$ & -0.496 & 9 \\
\hline 970 & $\mathrm{p}$ & $638-10-8$ & ethyl-3,3-dimethyl acrylate & $\mathrm{C}(\backslash \mathrm{C}(\mathrm{OCC})=\mathrm{O})=\mathrm{C}(\backslash \mathrm{C}) \mathrm{C}$ & -0.484 & 9 \\
\hline 971 & $\mathrm{p}$ & $20474-93-5$ & allyl crotonate & $\mathrm{C}(\mathrm{OCC}=\mathrm{C})(\backslash \mathrm{C}=\mathrm{C} \backslash \mathrm{C})=\mathrm{O}$ & -0.481 & 9 \\
\hline 972 & $\mathrm{p}$ & $10371-45-6$ & crotocic acid sec-butyl ester & $\mathrm{O}(\mathrm{C}(\backslash \mathrm{C}=\mathrm{C} \backslash \mathrm{C})=\mathrm{O})[\mathrm{C} @ \mathrm{H}](\mathrm{CC}) \mathrm{C}$ & -0.417 & 9 \\
\hline 973 & $\mathrm{p}$ & $1567-14-2$ & methyl-trans-2-methyl-2-pentenoate & $\mathrm{C}(\backslash \mathrm{C}(\mathrm{OC})=\mathrm{O})(=\mathrm{C} \backslash \mathrm{CC}) \mathrm{C}$ & -0.376 & 9 \\
\hline 974 & $\mathrm{p}$ & $13861-22-8$ & propargyl methacrylate & $\mathrm{C}(\mathrm{C}(\mathrm{C})=\mathrm{C})(\mathrm{OCC} \# \mathrm{C})=\mathrm{O}$ & -0.372 & 9 \\
\hline
\end{tabular}




\begin{tabular}{|c|c|c|c|c|c|c|}
\hline 975 & $\mathrm{p}$ & $565-62-8$ & 3-methyl-3-penten-2-one & $\mathrm{C}(\backslash \mathrm{C}(\mathrm{C})=\mathrm{O})(=\mathrm{C} \backslash \mathrm{C}) \mathrm{C}$ & -0.345 & 9 \\
\hline 976 & $\mathrm{p}$ & $589-66-2$ & crotonic acid isobutyl ester & $\mathrm{C}(\mathrm{OCC}(\mathrm{C}) \mathrm{C})(\backslash \mathrm{C}=\mathrm{C} \backslash \mathrm{C})=\mathrm{O}$ & -0.344 & 9 \\
\hline 977 & $\mathrm{p}$ & $97-86-9$ & isobutyl methacrylate & $\mathrm{C}(\mathrm{OCC}(\mathrm{C}) \mathrm{C})(\mathrm{C}(\mathrm{C})=\mathrm{C})=\mathrm{O}$ & -0.279 & 9 \\
\hline 978 & $\mathrm{p}$ & $97-88-1$ & butyl methacrylate & $\mathrm{C}(\mathrm{OCCCC})(\mathrm{C}(\mathrm{C})=\mathrm{C})=\mathrm{O}$ & -0.269 & 9 \\
\hline 979 & $\mathrm{p}$ & 7299-91-4 & crotonic acid $n$-butyl ester & $\mathrm{O}(\mathrm{C}(\backslash \mathrm{C}=\mathrm{C} \backslash \mathrm{C})=\mathrm{O}) \mathrm{CCCC}$ & -0.159 & 9 \\
\hline 980 & $\mathrm{p}$ & $497-03-0$ & trans-2-methyl-2-butenal & $\mathrm{C}(=\mathrm{C} \backslash \mathrm{C})(\backslash \mathrm{C}=\mathrm{O}) \mathrm{C}$ & -0.139 & 9 \\
\hline 981 & $\mathrm{p}$ & $623-36-9$ & 2-methyl-2-pentenal & $\mathrm{C}(=\mathrm{C} \backslash \mathrm{CC})(\backslash \mathrm{C}=\mathrm{O}) \mathrm{C}$ & -0.022 & 9 \\
\hline 982 & $\mathrm{p}$ & $85136-08-9$ & 2,4-dimethyl-2,6-heptadienal & $\mathrm{C}(\backslash \mathrm{C} @ @ @ \mathrm{H}](\mathrm{CC}=\mathrm{C}) \mathrm{C})=\mathrm{C}(\backslash \mathrm{C}=\mathrm{O}) \mathrm{C}$ & 0.083 & 9 \\
\hline 983 & $\mathrm{p}$ & $107-86-8$ & 3-methyl-2-butenal & $\mathrm{C}(=\mathrm{C}(\backslash \mathrm{C}) \mathrm{C}) \backslash \mathrm{C}=\mathrm{O}$ & 0.088 & 9 \\
\hline 984 & $\mathrm{p}$ & $106-63-8$ & isobutyl acrylate & $\mathrm{C}(\mathrm{OCC}(\mathrm{C}) \mathrm{C})(\mathrm{C}=\mathrm{C})=\mathrm{O}$ & 0.291 & 9 \\
\hline 985 & $\mathrm{p}$ & $23326-27-4$ & methyl-2-butynoate & $\mathrm{C}(\mathrm{C} \# \mathrm{CC})(\mathrm{OC})=\mathrm{O}$ & 0.403 & 9 \\
\hline 986 & $\mathrm{p}$ & $4245-35-6$ & isoamyl acrylate & $\mathrm{C}(\mathrm{OCCC}(\mathrm{C}) \mathrm{C})(\mathrm{C}=\mathrm{C})=\mathrm{O}$ & 0.408 & 9 \\
\hline 987 & $\mathrm{p}$ & $4341-76-8$ & ethyl-2-butynoate & $\mathrm{C}(\mathrm{C} \# \mathrm{CC})(\mathrm{OCC})=\mathrm{O}$ & 0.509 & 9 \\
\hline 988 & $\mathrm{p}$ & $140-88-5$ & ethyl acrylate & $\mathrm{C}(\mathrm{OCC})(\mathrm{C}=\mathrm{C})=\mathrm{O}$ & 0.516 & 9 \\
\hline 989 & $\mathrm{p}$ & $141-32-2$ & butyl acrylate & $\mathrm{C}(\mathrm{OCCCC})(\mathrm{C}=\mathrm{C})=\mathrm{O}$ & 0.519 & 9 \\
\hline 990 & $\mathrm{p}$ & $925-60-0$ & propyl acrylate & $\mathrm{C}(\mathrm{OCCC})(\mathrm{C}=\mathrm{C})=\mathrm{O}$ & 0.531 & 9 \\
\hline 991 & $\mathrm{p}$ & $2998-23-4$ & $n$-pentylacrylate & $\mathrm{C}(\mathrm{OCCCCC})(\mathrm{C}=\mathrm{C})=\mathrm{O}$ & 0.537 & 9 \\
\hline 992 & $\mathrm{p}$ & $625-33-2$ & 3-penten-2-one & $\mathrm{C}(\backslash \mathrm{C}(\mathrm{C})=\mathrm{O})=\mathrm{C} \backslash \mathrm{C}$ & 0.538 & 9 \\
\hline 993 & $\mathrm{p}$ & $96-33-3$ & methyl acrylate & $\mathrm{C}(\mathrm{C}=\mathrm{C})(\mathrm{OC})=\mathrm{O}$ & 0.547 & 9 \\
\hline 994 & $\mathrm{p}$ & $930-30-3$ & 2-cyclopenten-1-one & $\mathrm{O}=\mathrm{C} 1 \mathrm{CCC}=\mathrm{C} 1$ & 0.638 & 9 \\
\hline 995 & $\mathrm{p}$ & 999-61-1 & 2-hydroxypropyl acrylate & $\mathrm{C}(\mathrm{OC}[\mathrm{C} @ \mathrm{H}](\mathrm{C}) \mathrm{O})(\mathrm{C}=\mathrm{C})=\mathrm{O}$ & 0.65 & 9 \\
\hline 996 & $\mathrm{p}$ & $999-55-3$ & allyl acrylate & $\mathrm{C}(\mathrm{OCC}=\mathrm{C})(\mathrm{C}=\mathrm{C})=\mathrm{O}$ & 0.682 & 9 \\
\hline 997 & $\mathrm{p}$ & $1119-44-4$ & 3-hepten-2-one & $\mathrm{C}(=\mathrm{C} \backslash \mathrm{CCC}) \backslash \mathrm{C}(\mathrm{C})=\mathrm{O}$ & 0.698 & 9 \\
\hline 998 & $\mathrm{p}$ & $123-73-9$ & crotonaldehyde & $\mathrm{C}(=\mathrm{C} \backslash \mathrm{C}) \backslash \mathrm{C}=\mathrm{O}$ & 0.7 & 9 \\
\hline 999 & $\mathrm{p}$ & $16205-90-6$ & ethyl-2-hexynoate & $\mathrm{C}(\mathrm{C}(\mathrm{OCC})=\mathrm{O}) \# \mathrm{CCCC}$ & 0.7 & 9 \\
\hline 1000 & $\mathrm{p}$ & $1669-44-9$ & 3-octen-2-one & $\mathrm{C}(=\mathrm{C} \backslash \mathrm{C}(\mathrm{C})=\mathrm{O}) \backslash \mathrm{CCCC}$ & 0.74 & 9 \\
\hline 1001 & $\mathrm{p}$ & $2499-95-8$ & n-hexylacrylate & $\mathrm{C}(\mathrm{OC}(\mathrm{C}=\mathrm{C})=\mathrm{O}) \mathrm{CCCCC}$ & 0.741 & 9 \\
\hline 1002 & $\mathrm{p}$ & $142-83-6$ & 2,4-hexadienal & $\mathrm{C}(\backslash \mathrm{C}=\mathrm{C} \backslash \mathrm{C})=\mathrm{C} \backslash \mathrm{C}=\mathrm{O}$ & 0.754 & 9 \\
\hline 1003 & $\mathrm{p}$ & $18937-79-6$ & methyl-2-hexynoate & $\mathrm{C}(\mathrm{C} \# \mathrm{CCCC})(\mathrm{OC})=\mathrm{O}$ & 0.763 & 9 \\
\hline 1004 & $\mathrm{p}$ & $3066-71-5$ & cyclohexylacrylate & $\mathrm{O}(\mathrm{C}(\mathrm{C}=\mathrm{C})=\mathrm{O}) \mathrm{C} 1 \mathrm{CCCCC} 1$ & 0.763 & 9 \\
\hline 1005 & $\mathrm{p}$ & $7367-81-9$ & methyl-trans-2-octenoate & $\mathrm{C}(=\mathrm{C} \backslash \mathrm{C}(\mathrm{OC})=\mathrm{O}) \backslash \mathrm{CCCCC}$ & 0.764 & 9 \\
\hline 1006 & $\mathrm{p}$ & $5362-56-1$ & 4-methyl-2-pentenal & $\mathrm{C}(\backslash \mathrm{C}(\mathrm{C}) \mathrm{C})=\mathrm{C} \backslash \mathrm{C}=\mathrm{O}$ & 0.819 & 9 \\
\hline 1007 & $\mathrm{p}$ & $55314-57-3$ & ethyl 2-pentynoate & $\mathrm{C}(\mathrm{C} \# \mathrm{CCC})(\mathrm{OCC})=\mathrm{O}$ & 0.825 & 9 \\
\hline 1008 & $\mathrm{p}$ & $4313-03-5$ & trans,trans-2,4-heptadienal $^{C}$ & $\mathrm{C}(\backslash \mathrm{C}=\mathrm{C} \backslash \mathrm{C}=\mathrm{O})=\mathrm{C} \backslash \mathrm{CC}$ & 0.862 & 9 \\
\hline 1009 & $\mathrm{p}$ & $922-63-4$ & 2-ethylacrolein & $\mathrm{C}(\mathrm{CC})(\mathrm{C}=\mathrm{O})=\mathrm{C}$ & 0.911 & 9 \\
\hline 1010 & $\mathrm{p}$ & $2497-21-4$ & 4-hexen-3-one & $\mathrm{C}(\backslash \mathrm{C}=\mathrm{C} \backslash \mathrm{C})(\mathrm{CC})=\mathrm{O}$ & 0.93 & 9 \\
\hline 1011 & $\mathrm{p}$ & $14309-57-0$ & 3-nonen-2-one & $\mathrm{C}(\backslash \mathrm{CCCCC})=\mathrm{C} \backslash \mathrm{C}(\mathrm{C})=\mathrm{O}$ & 0.983 & 9 \\
\hline 141012 & $\mathrm{p}$ & $111-79-5$ & methyl-2-nonenoate & $\mathrm{C}(\backslash \mathrm{C}=\mathrm{C} \backslash \mathrm{C}(\mathrm{OC})=\mathrm{O}) \mathrm{CCCCC}$ & 1.039 & 9 \\
\hline 1013 & $\mathrm{p}$ & $111-12-6$ & methyl-2-octynoate & $\mathrm{C}(\# \mathrm{CC}(\mathrm{OC})=\mathrm{O}) \mathrm{CCCCC}$ & 1.043 & 9 \\
\hline
\end{tabular}




\begin{tabular}{|c|c|c|c|c|c|c|}
\hline 1014 & $\mathrm{p}$ & $1070-66-2$ & 2-butylacrolein & $\mathrm{C}(\mathrm{CCCC})(\mathrm{C}=\mathrm{O})=\mathrm{C}$ & 1.069 & 9 \\
\hline 1015 & $\mathrm{p}$ & $2499-58-3$ & $n$-heptyl acrylate & $\mathrm{C}(\mathrm{COC}(\mathrm{C}=\mathrm{C})=\mathrm{O}) \mathrm{CCCCC}$ & 1.086 & 9 \\
\hline 1016 & $\mathrm{p}$ & $142-09-6$ & $n$-hexyl methacrylate & $\mathrm{O}(\mathrm{C}(\mathrm{C}(\mathrm{C})=\mathrm{C})=\mathrm{O}) \mathrm{CCCCCC}$ & 1.087 & 9 \\
\hline 1017 & $\mathrm{p}$ & $16930-95-3$ & ethyl-2-heptynoate & $\mathrm{C}(\mathrm{C} \# \mathrm{CCCCC})(\mathrm{OCC})=\mathrm{O}$ & 1.106 & 9 \\
\hline 1018 & $\mathrm{p}$ & $19485-03-1$ & 1,3-butanediol diacrylate & $\begin{array}{l}\mathrm{C}([\mathrm{C} @ \mathrm{H}](\mathrm{OC}(\mathrm{C}=\mathrm{C})=\mathrm{O}) \mathrm{C}) \mathrm{COC}( \\
\mathrm{C}=\mathrm{C})=\mathrm{O}\end{array}$ & 1.144 & 9 \\
\hline 1019 & $\mathrm{p}$ & $5910-87-2$ & trans,trans-2,4-nonadienal & $\mathrm{C}(=\mathrm{C} \backslash \mathrm{C}=\mathrm{C} \backslash \mathrm{C}=\mathrm{O}) \backslash \mathrm{CCCC}$ & 1.225 & 9 \\
\hline 1020 & $\mathrm{p}$ & $10519-20-7$ & ethyl-2-octynoate & $\mathrm{C}(\mathrm{C} \# \mathrm{CCCCCC})(\mathrm{OCC})=\mathrm{O}$ & 1.233 & 9 \\
\hline 1021 & $\mathrm{p}$ & $1679-36-3$ & 3-hexyn-2-one & $\mathrm{C}(\mathrm{C}(\mathrm{C})=\mathrm{O}) \# \mathrm{CCC}$ & 1.319 & 9 \\
\hline 1022 & $\mathrm{p}$ & $111-80-8$ & methyl-2-nonynoate & $\mathrm{C}(\mathrm{C} \# \mathrm{CC}(\mathrm{OC})=\mathrm{O}) \mathrm{CCCCC}$ & 1.322 & 9 \\
\hline 1023 & $\mathrm{p}$ & $557-48-2$ & trans-2,cis-6-nonadienal & $\mathrm{C}(\mathrm{C} \backslash \mathrm{C}=\mathrm{C} \backslash \mathrm{C}=\mathrm{O}) \backslash \mathrm{C}=\mathrm{C} \backslash \mathrm{CC}$ & 1.335 & 9 \\
\hline 1024 & $\mathrm{p}$ & $1423-60-5$ & 3-butyn-2-one & $\mathrm{C}(\mathrm{C} \# \mathrm{C})(\mathrm{C})=\mathrm{O}$ & 1.412 & 9 \\
\hline 1025 & $\mathrm{p}$ & $78-94-4$ & 3-buten-2-one & $\mathrm{C}(\mathrm{C}=\mathrm{C})(\mathrm{C})=\mathrm{O}$ & 1.506 & 9 \\
\hline 1026 & $\mathrm{p}$ & $1629-58-9$ & 1-penten-3-one & $\mathrm{C}(\mathrm{CC})(\mathrm{C}=\mathrm{C})=\mathrm{O}$ & 1.527 & 9 \\
\hline 1027 & $\mathrm{p}$ & $688-84-6$ & 2-ethylhexyl methacrylate & $\begin{array}{l}\mathrm{C}([\mathrm{C} @ \mathrm{H}](\mathrm{CCCC}) \mathrm{CC}) \mathrm{OC}(\mathrm{C}(\mathrm{C})= \\
\mathrm{C})=\mathrm{O}\end{array}$ & 1.569 & 9 \\
\hline 1028 & $\mathrm{p}$ & $1629-60-3$ & 1-hexen-3-one & $\mathrm{C}(\mathrm{CCC})(\mathrm{C}=\mathrm{C})=\mathrm{O}$ & 1.656 & 9 \\
\hline 1029 & $\mathrm{p}$ & $3913-81-3$ & trans-2-decen-1-al & $\mathrm{C}(\mathrm{CCCCC}) \mathrm{C} \backslash \mathrm{C}=\mathrm{C} \backslash \mathrm{C}=\mathrm{O}$ & 1.851 & 9 \\
\hline 1030 & $\mathrm{p}$ & $107-02-8$ & acrolein & $\mathrm{C}(\mathrm{C}=\mathrm{O})=\mathrm{C}$ & 1.873 & 9 \\
\hline 1031 & $\mathrm{p}$ & $4312-99-6$ & 1-octen-3-one & $\mathrm{C}(\mathrm{CCCCC})(\mathrm{C}=\mathrm{C})=\mathrm{O}$ & 1.914 & 9 \\
\hline 1032 & $\mathrm{p}$ & $66-77-3$ & 1-Naphthaldehyde & $\mathrm{C}(=\mathrm{O}) \mathrm{c} 1 \mathrm{c} 2 \mathrm{c}(\operatorname{ccc} 1) \operatorname{ccc} 2$ & 0.423 & 10 \\
\hline 1033 & $\mathrm{p}$ & $3218-36-8$ & 4-Biphenylcarboxaldehyde & $\mathrm{C}(=\mathrm{O}) \mathrm{c} 1 \mathrm{ccc}(\mathrm{cc} 1) \mathrm{c} 1 \mathrm{ccccc} 1$ & 1.119 & 2,10 \\
\hline 1034 & $\mathrm{p}$ & $1122-91-4$ & 4-Bromobenzaldehyde & $\mathrm{C}(=\mathrm{O}) \mathrm{c} 1 \mathrm{ccc}(\mathrm{cc} 1) \mathrm{Br}$ & 0.587 & 10 \\
\hline 1035 & $\mathrm{p}$ & $104-87-0$ & $p$-Tolualdehyde & $\mathrm{C}(=\mathrm{O}) \mathrm{c} 1 \mathrm{ccc}(\mathrm{cc} 1) \mathrm{C}$ & -0.057 & 2,10 \\
\hline 1036 & $\mathrm{p}$ & $459-57-4$ & 4-Fluorobenzaldehyde & $\mathrm{C}(=\mathrm{O}) \mathrm{c} 1 \mathrm{ccc}(\mathrm{cc} 1) \mathrm{F}$ & -0.127 & 10 \\
\hline 1037 & $\mathrm{p}$ & $4748-78-1$ & 4-Ethylbenzaldehyde & $\mathrm{C}(\mathrm{C}) \mathrm{c} 1 \mathrm{ccc}(\mathrm{cc} 1) \mathrm{C}=\mathrm{O}$ & 0.291 & 2,10 \\
\hline 1038 & $\mathrm{p}$ & $623-27-8$ & Terephthaldicarboxaldehyde & $\mathrm{C}(=\mathrm{O}) \mathrm{c} 1 \operatorname{ccc}(\operatorname{cc} 1) \mathrm{C}=\mathrm{O}$ & -0.086 & 10 \\
\hline 1039 & $\mathrm{p}$ & $123-11-5$ & 4-Anisaldehyde & $\mathrm{C}(=\mathrm{O}) \mathrm{c} 1 \mathrm{ccc}(\mathrm{cc} 1) \mathrm{OC}$ & -0.047 & 10 \\
\hline 1040 & $\mathrm{p}$ & $10031-82-0$ & 4-Ethoxybenzaldehyde & $\mathrm{O}(\mathrm{CC}) \mathrm{c} 1 \operatorname{ccc}(\operatorname{cc} 1) \mathrm{C}=\mathrm{O}$ & 0.073 & 2,10 \\
\hline 1041 & $\mathrm{p}$ & $122-85-0$ & 4-Acetamidobenzaldehyde & $\mathrm{N}(\mathrm{C}(\mathrm{C})=\mathrm{O}) \mathrm{c} 1 \operatorname{ccc}(\mathrm{cc} 1) \mathrm{C}=\mathrm{O}$ & -0.224 & 10 \\
\hline 1042 & $\mathrm{p}$ & $529-20-4$ & 2-Tolualdehyde & $\mathrm{C}(=\mathrm{O}) \mathrm{c} 1 \mathrm{c}(\mathrm{C}) \mathrm{cccc} 1$ & 0.011 & 2 \\
\hline 1043 & $\mathrm{p}$ & $620-23-5$ & 3-Tolualdehyde & $\mathrm{C}(=\mathrm{O}) \mathrm{c} 1 \mathrm{cc}(\mathrm{C}) \mathrm{ccc} 1$ & 0.081 & 10 \\
\hline 1044 & $\mathrm{p}$ & $89-98-5$ & 2-Chlorobenzaldehyde & $\mathrm{C}(=\mathrm{O}) \mathrm{c} 1 \mathrm{c}(\mathrm{Cl}) \operatorname{cccc} 1$ & 0.487 & 10 \\
\hline 1045 & $\mathrm{p}$ & $587-04-2$ & 3-Chlorobenzaldehyde & $\mathrm{C}(=\mathrm{O}) \mathrm{c} 1 \mathrm{cc}(\mathrm{Cl}) \operatorname{ccc} 1$ & 0.406 & 10 \\
\hline 1046 & $\mathrm{p}$ & $626-19-7$ & 1,3-Benzenedialdehyde & $\mathrm{C}(=\mathrm{O}) \mathrm{c} 1 \mathrm{cc}(\mathrm{C}=\mathrm{O}) \mathrm{ccc} 1$ & 0.183 & 10 \\
\hline 1047 & $\mathrm{p}$ & $135-02-4$ & 2-Anisaldehyde & $\mathrm{C}(=\mathrm{O}) \mathrm{c} 1 \mathrm{c}(\mathrm{OC}) \mathrm{cccc} 1$ & 0.148 & 10 \\
\hline 1048 & $\mathrm{p}$ & $591-31-1$ & 3-Methoxybenzaldehyde & $\mathrm{C}(=\mathrm{O}) \mathrm{c} 1 \mathrm{cc}(\mathrm{OC}) \mathrm{ccc} 1$ & 0.232 & 10 \\
\hline 1049 & $\mathrm{p}$ & $3132-99-8$ & 3-Bromobenzaldehyde & $\mathrm{C}(=\mathrm{O}) \mathrm{c} 1 \mathrm{cc}(\mathrm{Br}) \mathrm{ccc} 1$ & 0.506 & 10 \\
\hline 1050 & $\mathrm{p}$ & $456-48-4$ & 3-Fluorobenzaldehyde & $\mathrm{C}(=\mathrm{O}) \mathrm{c} 1 \mathrm{cc}(\mathrm{F}) \mathrm{ccc} 1$ & 0.154 & 10 \\
\hline
\end{tabular}




\begin{tabular}{|c|c|c|c|c|c|c|}
\hline 1051 & $\mathrm{p}$ & $874-42-0$ & 2,4-Dichlorobenzaldehyde & $\mathrm{C}(=\mathrm{O}) \mathrm{c} 1 \mathrm{c}(\mathrm{Cl}) \mathrm{cc}(\mathrm{cc} 1) \mathrm{Cl}$ & 1.036 & 2,10 \\
\hline 1052 & $\mathrm{p}$ & $613-45-6$ & 2,4-Dimethoxybenzaldehyde & $\mathrm{O}(\mathrm{C}) \mathrm{c} 1 \mathrm{c}(\mathrm{C}=\mathrm{O}) \operatorname{ccc}(\mathrm{c} 1) \mathrm{OC}$ & -0.056 & 2,10 \\
\hline 1053 & $\mathrm{p}$ & $4460-86-0$ & 2,4,5-Trimethoxybenzaldehyde & $\mathrm{C}(=\mathrm{O}) \mathrm{c} 1 \mathrm{c}(\mathrm{OC}) \mathrm{cc}(\mathrm{c}(\mathrm{c} 1) \mathrm{OC}) \mathrm{OC}$ & -0.101 & 2,10 \\
\hline 1054 & $\mathrm{p}$ & $100-10-7$ & 4-(Dimethylamino)Benzaldehyde & $\mathrm{N}(\mathrm{C})(\mathrm{C}) \mathrm{c} 1 \mathrm{ccc}(\operatorname{cc} 1) \mathrm{C}=\mathrm{O}$ & 0.231 & 2,10 \\
\hline 1055 & $\mathrm{p}$ & $67-36-7$ & 4-Phenoxybenzaldehyde & $\mathrm{O}(\mathrm{c} 1 \mathrm{ccc}(\mathrm{cc} 1) \mathrm{C}=\mathrm{O}) \mathrm{c} 1 \mathrm{ccccc} 1$ & 1.257 & 2,10 \\
\hline 1056 & $\mathrm{p}$ & $6630-33-7$ & 2-Bromobenzaldehyde & $\mathrm{C}(=\mathrm{O}) \mathrm{c} 1 \mathrm{c}(\mathrm{Br}) \mathrm{cccc} 1$ & 0.477 & 10 \\
\hline 1057 & $\mathrm{p}$ & $446-52-6$ & 2-Fluorobenzaldehyde & $\mathrm{C}(=\mathrm{O}) \mathrm{c} 1 \mathrm{c}(\mathrm{F}) \mathrm{cccc} 1$ & 0.079 & 10 \\
\hline 1058 & $\mathrm{p}$ & $5736-88-9$ & 4-Butoxybenzaldehyde & $\mathrm{O}(\mathrm{CCCC}) \mathrm{c} 1 \operatorname{ccc}(\operatorname{cc} 1) \mathrm{C}=\mathrm{O}$ & 0.716 & 10 \\
\hline 1059 & $\mathrm{p}$ & $6361-22-4$ & 2-Chloro-6-nitrobenzaldehyde & $\mathrm{C}(=\mathrm{O}) \mathrm{c} 1 \mathrm{c}(\mathrm{N}(=\mathrm{O})=\mathrm{O}) \operatorname{ccc} 1 \mathrm{Cl}$ & 0.155 & 10 \\
\hline 1060 & $\mathrm{p}$ & $37687-57-3$ & $\begin{array}{l}\text { 2-Chloro-3-hydroxy-4-methoxybenzald } \\
\text { ehyde }\end{array}$ & $\mathrm{C}(=\mathrm{O}) \mathrm{c} 1 \mathrm{c}(\mathrm{Cl}) \mathrm{c}(\mathrm{O}) \mathrm{c}(\mathrm{cc} 1) \mathrm{OC}$ & 0.204 & 10 \\
\hline 1061 & $\mathrm{p}$ & $286474-59-7$ & $\begin{array}{l}\text { 6-Chloro-2-fluoro-3-methylbenzaldehy } \\
\text { de }\end{array}$ & $\mathrm{C}(=\mathrm{O}) \mathrm{c} 1 \mathrm{c}(\mathrm{F}) \mathrm{c}(\mathrm{C}) \mathrm{ccc} 1 \mathrm{Cl}$ & 1.238 & 10 \\
\hline 1062 & $\mathrm{p}$ & $261763-02-4$ & $\begin{array}{l}\text { 3-Chloro-2-fluoro-5-(trifluoromethyl)b } \\
\text { enzaldehyde }\end{array}$ & $\mathrm{C}(\mathrm{F})(\mathrm{F})(\mathrm{F}) \mathrm{c} 1 \mathrm{cc}(\mathrm{C}=\mathrm{O}) \mathrm{c}(\mathrm{c}(\mathrm{c} 1) \mathrm{Cl}) \mathrm{F}$ & 1.723 & 10 \\
\hline 1063 & $\mathrm{p}$ & $56961-75-2$ & 2,3,5-Trichlorobenzaldehyde & $\mathrm{C}(=\mathrm{O}) \mathrm{clc}(\mathrm{Cl}) \mathrm{c}(\mathrm{Cl}) \mathrm{cc}(\mathrm{cl}) \mathrm{Cl}$ & 1.499 & 10 \\
\hline 1064 & $\mathrm{p}$ & $30084-90-3$ & Fluorene-2-carbaldehyde & $\mathrm{C}(=\mathrm{O}) \mathrm{c} 1 \mathrm{cc} 2 \mathrm{c}(\mathrm{cc} 1) \mathrm{c} 1 \mathrm{c}(\mathrm{C} 2) \operatorname{ccc} 1$ & 1.499 & 10 \\
\hline 1065 & $\mathrm{p}$ & $35699-44-6$ & 2-Methyl-1-naphthaldehyde & $\mathrm{C}(=\mathrm{O}) \mathrm{c} 1 \mathrm{c} 2 \mathrm{c}(\operatorname{ccc} 1 \mathrm{C}) \operatorname{ccc} 2$ & 1.231 & 10 \\
\hline 1066 & $\mathrm{p}$ & $33738-48-6$ & $\begin{array}{l}\text { 4-Methylnaphthalene-1-carboxaldehyd } \\
\text { e }\end{array}$ & $\mathrm{C}(=\mathrm{O}) \mathrm{c} 1 \mathrm{c} 2 \mathrm{c}(\mathrm{c}(\mathrm{cc} 1) \mathrm{C}) \mathrm{cccc} 2$ & 1.123 & 10 \\
\hline 1067 & $\mathrm{p}$ & $4707-71-5$ & 9-Phenanthrenecarbaldehyde & $\mathrm{C}(=\mathrm{O}) \mathrm{c} 1 \mathrm{c} 2 \mathrm{c}(\mathrm{c} 3 \mathrm{c}(\mathrm{c} 1) \operatorname{ccc} 3) \operatorname{ccc} 2$ & 1.708 & 10 \\
\hline 1068 & $\mathrm{p}$ & $29865-90-5$ & $\begin{array}{l}\text { 3,4-Dimethoxy-5-hydroxybenzaldehyd } \\
\text { e }\end{array}$ & $\mathrm{O}(\mathrm{C}) \mathrm{c} 1 \mathrm{c}(\mathrm{OC}) \mathrm{c}(\mathrm{O}) \mathrm{cc}(\mathrm{c} 1) \mathrm{C}=\mathrm{O}$ & -0.390 & 10 \\
\hline 1069 & $\mathrm{p}$ & $24677-78-9$ & 2,3-Dihydroxybenzaldehyde & $\mathrm{C}(=\mathrm{O}) \mathrm{c} 1 \mathrm{c}(\mathrm{O}) \mathrm{c}(\mathrm{O}) \mathrm{ccc} 1$ & 0.111 & 13 \\
\hline 1070 & $\mathrm{p}$ & $1194-98-5$ & 2,5-Dihydroxybenzaldehyde & $\mathrm{C}(=\mathrm{O}) \mathrm{c} 1 \mathrm{c}(\mathrm{O}) \operatorname{ccc}(\mathrm{c} 1) \mathrm{O}$ & 0.277 & 10,13 \\
\hline 1071 & $\mathrm{p}$ & $139-85-5$ & 3,4-Dihydroxybenzaldehyde & $\mathrm{C}(=\mathrm{O}) \mathrm{c} 1 \mathrm{cc}(\mathrm{O}) \mathrm{c}(\mathrm{cc} 1) \mathrm{O}$ & 0.107 & 10 \\
\hline 1072 & $\mathrm{p}$ & $13677-79-7$ & 3,4,5-Trihydroxybenzaldehyde & $\mathrm{C}(=\mathrm{O}) \mathrm{c} 1 \mathrm{cc}(\mathrm{O}) \mathrm{c}(\mathrm{c}(\mathrm{c} 1) \mathrm{O}) \mathrm{O}$ & -0.196 & 10 \\
\hline 1073 & $\mathrm{p}$ & $2144-08-3$ & 2,3,4-Trihydroxybenzaldehyde & $\mathrm{C}(=\mathrm{O}) \mathrm{c} 1 \mathrm{c}(\mathrm{O}) \mathrm{c}(\mathrm{O}) \mathrm{c}(\mathrm{cc} 1) \mathrm{O}$ & 0.001 & 10 \\
\hline 1074 & $\mathrm{p}$ & $487-70-7$ & 2,4,6-Trihydroxybenzaldehyde & $\mathrm{C}(=\mathrm{O}) \mathrm{c} 1 \mathrm{c}(\mathrm{O}) \mathrm{cc}(\mathrm{cc} 1 \mathrm{O}) \mathrm{O}$ & 0.128 & 10 \\
\hline 1075 & $\mathrm{p}$ & $95-01-2$ & 2,4-Dihydroxybenzaldehyde & $\mathrm{C}(=\mathrm{O}) \mathrm{c} 1 \mathrm{c}(\mathrm{O}) \mathrm{cc}(\mathrm{cc} 1) \mathrm{O}$ & 0.515 & 2,10 \\
\hline 1076 & $\mathrm{p}$ & $492-88-6$ & 3-Ethoxy-2-hydroxybenzaldehyde & $\mathrm{O}(\mathrm{CC}) \mathrm{c} 1 \mathrm{c}(\mathrm{O}) \mathrm{c}(\mathrm{C}=\mathrm{O}) \mathrm{ccc} 1$ & 0.85 & 10 \\
\hline 1077 & $\mathrm{p}$ & $708-76-9$ & $\begin{array}{l}\text { 4,6-Dimethoxy-2-hydroxybenzaldehyd } \\
\text { e }\end{array}$ & $\mathrm{O}(\mathrm{C}) \mathrm{c} 1 \mathrm{c}(\mathrm{C}=\mathrm{O}) \mathrm{c}(\mathrm{O}) \mathrm{cc}(\mathrm{c} 1) \mathrm{OC}$ & 0.617 & 10 \\
\hline 1078 & $\mathrm{p}$ & $5274-70-4$ & 2-Hydroxy-3-nitrobenzaldehyde & $\mathrm{N}(=\mathrm{O})(=\mathrm{O}) \mathrm{c} 1 \mathrm{c}(\mathrm{O}) \mathrm{c}(\mathrm{C}=\mathrm{O}) \mathrm{ccc} 1$ & 0.87 & 10 \\
\hline 1079 & $\mathrm{p}$ & $56962-11-9$ & 2-Chloro-4-hydroxybenzaldehyde & $\mathrm{C}(=\mathrm{O}) \mathrm{c} 1 \mathrm{c}(\mathrm{Cl}) \mathrm{cc}(\mathrm{cc} 1) \mathrm{O}$ & 0.89 & 10 \\
\hline 1080 & $\mathrm{p}$ & $708-06-5$ & 2-Hydroxy-1-naphthaldehyde & $\mathrm{C}(=\mathrm{O}) \mathrm{c} 1 \mathrm{c} 2 \mathrm{c}(\operatorname{ccc} 1 \mathrm{O}) \operatorname{ccc} 2$ & 1.32 & 10 \\
\hline 1081 & $\mathrm{p}$ & $7770-45-8$ & 4-Hydroxy-1-naphthaldehyde & $\mathrm{C}(=\mathrm{O}) \mathrm{c} 1 \mathrm{c} 2 \mathrm{c}(\mathrm{c}(\mathrm{cc} 1) \mathrm{O}) \mathrm{cccc} 2$ & 1.05 & 10 \\
\hline 1082 & $\mathrm{p}$ & $1761-61-1$ & 5-Bromosalicylaldehyde & $\mathrm{C}(=\mathrm{O}) \mathrm{c} 1 \mathrm{c}(\mathrm{O}) \mathrm{ccc}(\mathrm{c} 1) \mathrm{Br}$ & 1.107 & 10 \\
\hline 1083 & $\mathrm{p}$ & $635-93-8$ & 5-Chlorosalicylaldehyde & $\mathrm{C}(=\mathrm{O}) \mathrm{c} 1 \mathrm{c}(\mathrm{O}) \mathrm{ccc}(\mathrm{c} 1) \mathrm{Cl}$ & 1.009 & 10 \\
\hline 1084 & $\mathrm{p}$ & $2973-77-5$ & 3,5-Dibromo-4-hydroxybenzaldehyde & $\mathrm{C}(=\mathrm{O}) \mathrm{c} 1 \mathrm{cc}(\mathrm{Br}) \mathrm{c}(\mathrm{c}(\mathrm{c} 1) \mathrm{Br}) \mathrm{O}$ & 0.89 & 10 \\
\hline
\end{tabular}




\begin{tabular}{|c|c|c|c|c|c|c|}
\hline 1085 & $\mathrm{n}$ & $137-40-6$ & Propionic acid sodium salt & $\mathrm{C}(\mathrm{CC})(=\mathrm{O}) \mathrm{O}$ & -1.45 & 6 \\
\hline 1086 & $\mathrm{p}$ & $16230-28-7$ & 3,4-Dibromohexane & $\mathrm{C}([\mathrm{C} @ \mathrm{H}]([\mathrm{C} @ @ \mathrm{H}](\mathrm{CC}) \mathrm{Br}) \mathrm{Br}) \mathrm{C}$ & 0.99 & 6 \\
\hline 1087 & $\mathrm{p}$ & $17346-16-6$ & 2,4-Dibromo-2,4-dimethyl-3-pentanone & $\mathrm{C}(\mathrm{C}(\mathrm{Br})(\mathrm{C}) \mathrm{C})(\mathrm{C}(\mathrm{Br})(\mathrm{C}) \mathrm{C})=\mathrm{O}$ & 0.51 & 6 \\
\hline 1088 & $\mathrm{p}$ & $17696-11-6$ & 8-Bromooctanoic acid & $\mathrm{C}(\mathrm{CCCCBr}) \mathrm{CCC}(=\mathrm{O}) \mathrm{O}$ & 1.32 & 6 \\
\hline 1089 & $\mathrm{p}$ & $20965-27-9$ & 7-Bromoheptanonitrile & $\mathrm{C}(\mathrm{CCCBr}) \mathrm{CCC} \# \mathrm{~N}$ & 0.51 & 6 \\
\hline 1090 & $\mathrm{p}$ & $22819-91-6$ & 7-Chloroheptanonitrile & $\mathrm{C}(\mathrm{CCCCl}) \mathrm{CCC} \# \mathrm{~N}$ & 0.287 & 6 \\
\hline 1091 & $\mathrm{p}$ & $23144-52-7$ & 8-Chloro-1-octanol & $\mathrm{C}(\mathrm{CCCCO}) \mathrm{CCCCl}$ & 0.488 & 6,12 \\
\hline 1092 & $\mathrm{n}$ & $34485-37-5$ & 2-Butynyl acetate & $\mathrm{O}(\mathrm{CC} \# \mathrm{CC}) \mathrm{C}(\mathrm{C})=\mathrm{O}$ & -0.883 & 6 \\
\hline 1093 & $\mathrm{p}$ & $39081-91-9$ & 2,5-Dibromo-3,4-hexanedione & $\begin{array}{l}\mathrm{C}(\mathrm{C}([\mathrm{C} @ \mathrm{H}](\mathrm{Br}) \mathrm{C})=\mathrm{O})([\mathrm{C} @ \mathrm{H}]( \\
\mathrm{Br}) \mathrm{C})=\mathrm{O}\end{array}$ & 2.168 & 6 \\
\hline 1094 & $\mathrm{p}$ & $5314-31-8$ & 2-Heptanone oxime & $\mathrm{C}(\mathrm{ICCCCC})(=\mathrm{N} / \mathrm{O}) \mathrm{C}$ & 0.103 & 6 \\
\hline 1095 & $\mathrm{p}$ & $5445-29-4$ & Ethyl 2-bromooctanoate & $\mathrm{C}([\mathrm{C} @ \mathrm{H}](\mathrm{CCCCCC}) \mathrm{Br})(\mathrm{OCC})=\mathrm{O}$ & 1.50 & 15 \\
\hline 1096 & $\mathrm{p}$ & $6175-23-1$ & 2,4-Nonanedione & $\mathrm{C}(\mathrm{C}(\mathrm{CC}(\mathrm{C})=\mathrm{O})=\mathrm{O}) \mathrm{CCCC}$ & 0.506 & 6 \\
\hline 1097 & $\mathrm{p}$ & $6305-43-7$ & 1,4-Dibromo-2,3-butanedione & $\mathrm{C}(\mathrm{C}(\mathrm{CBr})=\mathrm{O})(\mathrm{CBr})=\mathrm{O}$ & 1.762 & 6 \\
\hline 1098 & $\mathrm{p}$ & $75039-84-8$ & trans-2-Undecen-1-ol & $\mathrm{C}(\mathrm{CC} \backslash \mathrm{C}=\mathrm{C} \backslash \mathrm{CO}) \mathrm{CCCCC}$ & 1.611 & 6 \\
\hline 1099 & $\mathrm{n}$ & $763-32-6$ & 2-Methyl-1-buten-4-ol & $\mathrm{C}(\mathrm{C}(\mathrm{C})=\mathrm{C}) \mathrm{CO}$ & -1.185 & 6 \\
\hline 1100 & $\mathrm{p}$ & $87777-46-6$ & 4-Methyl-1-heptyn-3-ol & $\mathrm{C}([\mathrm{C} @ \mathrm{H}]([\mathrm{C} @ @ \mathrm{H}](\mathrm{C} \# \mathrm{C}) \mathrm{O}) \mathrm{C}) \mathrm{CC}$ & 0.743 & 6,12 \\
\hline 1101 & $\mathrm{n}$ & $98-37-3$ & $\begin{array}{l}\text { 3-Amino-4-hydroxybenzenesulfonic } \\
\text { acid }\end{array}$ & $\mathrm{S}(=\mathrm{O})(=\mathrm{O})(\mathrm{O}) \mathrm{c} 1 \mathrm{cc}(\mathrm{N}) \mathrm{c}(\mathrm{cc} 1) \mathrm{O}$ & -1.5 & 6 \\
\hline 1102 & $\mathrm{p}$ & $88-89-1$ & 2,4,6-trinitrophenol & $\begin{array}{l}\mathrm{N}(=\mathrm{O})(=\mathrm{O}) \mathrm{c} 1 \mathrm{c}(\mathrm{O}) \mathrm{c}(\mathrm{N}(=\mathrm{O})=\mathrm{O}) \mathrm{cc}(\mathrm{c} 1 \\
\mathrm{N}(=\mathrm{O})=\mathrm{O}\end{array}$ & -0.16 & 6 \\
\hline 1103 & $\mathrm{p}$ & $603-71-4$ & 1,3,5-trimethyl-2-nitrobenzene & $\mathrm{N}(=\mathrm{O})(=\mathrm{O}) \mathrm{c} 1 \mathrm{c}(\mathrm{C}) \operatorname{cc}(\mathrm{cc} 1 \mathrm{C}) \mathrm{C}$ & 0.86 & 1 \\
\hline 1104 & $\mathrm{p}$ & $610-39-9$ & 5-methyl-1,2-dinitrobenzene & $\mathrm{N}(=\mathrm{O})(=\mathrm{O}) \mathrm{clc}(\mathrm{N}(=\mathrm{O})=\mathrm{O}) \mathrm{cc}(\mathrm{cc} 1) \mathrm{C}$ & 1.52 & 3 \\
\hline 1105 & $\mathrm{p}$ & $2835-99-6$ & 2-Methyl-4-hydroxyaniline & $\mathrm{Cc} 1 \mathrm{c}(\mathrm{N}) \operatorname{ccc}(\mathrm{c} 1) \mathrm{O}$ & 1.31 & 3 \\
\hline 1106 & $\mathrm{p}$ & 2920-38-9 & 4-Biphenylnitrile & $\mathrm{C}(\# \mathrm{~N}) \mathrm{c} 1 \mathrm{ccc}(\mathrm{cc} 1) \mathrm{c} 1 \mathrm{ccccc} 1$ & 1.24 & 3 \\
\hline 1107 & $\mathrm{p}$ & $7244-78-2$ & 4-n-butoxynitrobenzene & $\mathrm{O}(\mathrm{CCCC}) \mathrm{c} 1 \operatorname{ccc}(\operatorname{cc} 1) \mathrm{N}(=\mathrm{O})=\mathrm{O}$ & 1.42 & 2,3 \\
\hline 1108 & $\mathrm{p}$ & $24065-40-5$ & $\begin{array}{l}\text { Carbanilic acid, } \\
\text { 2,5-dimethyl-4-thiocyanato-, } \\
\text { 3,4,5,6-tetrabromo-o-tolyl ester (8CI) }\end{array}$ & $\begin{array}{l}\mathrm{O}(\mathrm{C}(\mathrm{Nc} 1 \mathrm{c}(\mathrm{C}) \mathrm{cc}(\mathrm{c}(\mathrm{c} 1) \mathrm{C}) \mathrm{SC} \# \mathrm{~N})=\mathrm{O}) \mathrm{c} \\
1 \mathrm{c}(\mathrm{Br}) \mathrm{c}(\mathrm{Br}) \mathrm{c}(\mathrm{c}(\mathrm{c} 1 \mathrm{C}) \mathrm{Br}) \mathrm{Br}\end{array}$ & 2.57 & 3 \\
\hline 1109 & $\mathrm{p}$ & $101349-82-0$ & 4-Methyl-3-cyanophenol & $\mathrm{C}(\# \mathrm{~N}) \mathrm{c} 1 \mathrm{c}(\mathrm{C}) \operatorname{ccc}(\mathrm{c} 1) \mathrm{O}$ & -0.38 & 3 \\
\hline 1110 & $\mathrm{p}$ & $105891-30-3$ & $\begin{array}{l}\text { 2,3,4,5-Tetrachloro-1-nitrobenzene } \\
\text { anion radical }\end{array}$ & $\mathrm{N}(=\mathrm{O})(=\mathrm{O}) \mathrm{c} 1 \mathrm{c}(\mathrm{Cl}) \mathrm{c}(\mathrm{Cl}) \mathrm{c}(\mathrm{c}(\mathrm{c} 1) \mathrm{Cl}) \mathrm{Cl}$ & 1.78 & 3 \\
\hline 1111 & $\mathrm{p}$ & $147218-98-2$ & 2-hydroxy-4,5-dimethylacetophenone & $\begin{array}{l}\mathrm{C}(=\mathrm{N} / \mathrm{NC}(\mathrm{NC})=\mathrm{S})(\backslash \mathrm{C}) \mathrm{c} 1 \mathrm{c}(\mathrm{O}) \mathrm{cc}(\mathrm{c}(\mathrm{c} \\
1) \mathrm{C}) \mathrm{C}\end{array}$ & 0.71 & 3 \\
\hline 1112 & $\mathrm{p}$ & $95-81-8$ & 2-Chloro-5-methylbenzenamine & $\mathrm{Nclc}(\mathrm{Cl}) \operatorname{ccc}(\mathrm{c} 1) \mathrm{C}$ & 0.24 & 16 \\
\hline 1113 & $\mathrm{p}$ & $87-62-7$ & 2,6-Dimethylaniline & $\mathrm{Nclc}(\mathrm{C}) \operatorname{cccc} 1 \mathrm{C}$ & -0.27 & 16 \\
\hline 1114 & $\mathrm{p}$ & $13214-66-9$ & 4-Phenyl-1-butylamine & $\mathrm{C}(\mathrm{CCCN}) \mathrm{c} 1 \mathrm{ccccc} 1$ & 0.33 & 16 \\
\hline 1115 & $\mathrm{p}$ & $607-81-8$ & Diethyl benzylmalonate & $\begin{array}{l}\mathrm{C}(\mathrm{C}(\mathrm{OCC})=\mathrm{O})(\mathrm{C}(\mathrm{OCC})=\mathrm{O}) \mathrm{Cc} 1 \mathrm{cccc} \\
\mathrm{c} 1\end{array}$ & 0.31 & 16 \\
\hline 1116 & $\mathrm{p}$ & $582-22-9$ & 2-Phenylpropylamine & $\mathrm{C}([\mathrm{C} @ \mathrm{H}](\mathrm{C}) \mathrm{c} 1 \operatorname{ccccc} 1) \mathrm{N}$ & -0.05 & 16 \\
\hline
\end{tabular}




\begin{tabular}{|c|c|c|c|c|c|c|}
\hline 1117 & $\mathrm{p}$ & $5446-02-6$ & Methyl 4-methoxysalicylate & $\mathrm{C}(\mathrm{OC})(=\mathrm{O}) \mathrm{c} 1 \mathrm{c}(\mathrm{O}) \mathrm{cc}(\mathrm{cc} 1) \mathrm{OC}$ & 0.37 & $4,5,7,8,11$ \\
\hline 1118 & $\mathrm{p}$ & $103-84-4$ & N-Phenylacetamide & $\mathrm{N}(\mathrm{C}(\mathrm{C})=\mathrm{O}) \mathrm{c} 1 \operatorname{ccccc} 1$ & 0.40 & 2 \\
\hline 1119 & $\mathrm{p}$ & $106-50-3$ & 1,4-Phenylenediamine & Nclecc(cc1)N & -0.15 & 2 \\
\hline 1120 & $\mathrm{p}$ & $363-03-1$ & Phenyl-1,4-benzoquinone & $\mathrm{O}=\mathrm{C} 1 \mathrm{C}(=\mathrm{CC}(\mathrm{C}=\mathrm{C} 1)=\mathrm{O}) \mathrm{c} 1 \mathrm{ccccc} 1$ & 0.85 & 2 \\
\hline 1121 & $\mathrm{p}$ & $830-81-9$ & 1-Naphthyl acetate & $\mathrm{O}(\mathrm{C}(\mathrm{C})=\mathrm{O}) \mathrm{c} 1 \mathrm{c} 2 \mathrm{c}(\mathrm{ccc} 1) \operatorname{ccc} 2$ & 1.04 & 2 \\
\hline 1122 & $\mathrm{p}$ & $536-74-3$ & Phenylacetylene & $\mathrm{C}(\# \mathrm{C}) \mathrm{c} 1 \mathrm{ccccc} 1$ & 0.01 & 2 \\
\hline 1123 & $\mathrm{p}$ & $524-42-5$ & 1,2-Naphthoquinone & $\mathrm{O}=\mathrm{C} 1 \mathrm{c} 2 \mathrm{c}(\mathrm{C}=\mathrm{CC} 1=\mathrm{O}) \operatorname{ccc} 2$ & 1.10 & 2 \\
\hline 1124 & $\mathrm{p}$ & $536-60-7$ & 4-Isopropylbenzyl alcohol & $\mathrm{C}(\mathrm{C})(\mathrm{C}) \mathrm{c} 1 \mathrm{ccc}(\mathrm{cc} 1) \mathrm{CO}$ & 0.36 & 2 \\
\hline 1125 & $\mathrm{p}$ & $766-51-8$ & 2-Chloromethoxybenzene & $\mathrm{O}(\mathrm{C}) \mathrm{c} 1 \mathrm{c}(\mathrm{Cl}) \operatorname{cccc} 1$ & 0.40 & 2,3 \\
\hline 1126 & $\mathrm{p}$ & $87-25-2$ & Ethyl 2-aminobenzoate & $\mathrm{C}(\mathrm{OCC})(=\mathrm{O}) \mathrm{c} 1 \mathrm{c}(\mathrm{N}) \operatorname{cccc} 1$ & 0.15 & 2 \\
\hline 1127 & $\mathrm{p}$ & $635-12-1$ & 1,4-Anthraquinone & $\begin{array}{l}\mathrm{O}=\mathrm{C} 1 \mathrm{c} 2 \mathrm{c}(\mathrm{cc} 3 \mathrm{c}(\mathrm{c} 2) \operatorname{ccc} 3) \mathrm{C}(\mathrm{C}=\mathrm{C} 1)= \\
\mathrm{O}\end{array}$ & 2.31 & 2 \\
\hline 1128 & $\mathrm{p}$ & $98-06-6$ & tert-Butylbenzene & $\mathrm{C}(\mathrm{C})(\mathrm{C})(\mathrm{C}) \mathrm{c} 1 \mathrm{ccccc} 1$ & 0.76 & 2 \\
\hline 1129 & $\mathrm{p}$ & $92-92-2$ & p-Biphenylcarboxylic acid & $\mathrm{C}(=\mathrm{O})(\mathrm{O}) \mathrm{c} 1 \mathrm{ccc}(\mathrm{cc} 1) \mathrm{c} 1 \mathrm{cccc} 1$ & 0.84 & $3,4,5,7,8,16$ \\
\hline
\end{tabular}

\section{References:}

(1) Schultz, T. (1999) Structure-toxicity relationships for benzenes evaluated with Tetrahymena pyriformis. Chem. Res. Toxicol. 12, 1262-1267.

(2) Serra, J., Jurs, P. and Kaiser, K. (2001) Linear regression and computational neural network prediction of tetrahymena acute toxicity for aromatic compounds from molecular structure. Chem. Res. Toxicol. 14, 1535-1545.

(3) Cronin, M. and Schultz, T. (2001) Development of quantitative structure-activity relationships for the toxicity of aromatic compounds to Tetrahymena pyriformis: comparative assessment of the methodologies. Chem. Res. Toxicol. 14, $1284-1295$.

(4) Cronin, M., Aptula, A., Duffy, J., Netzeva, T., Rowe, P., Valkova, I. and Schultz, T. (2002) Comparative assessment of methods to develop QSARs for the prediction of the toxicity of phenols to Tetrahymena pyriformis. Chemosphere 49, $1201-1221$.

(5) Schuurmann, G., Aptula, A., Kuhne, R. and Ebert, R. (2003) Stepwise discrimination between four modes of toxic action of phenols in the Tetrahymena pyriformis assay. Chem. Res. Toxicol. 16, 974-987.

(6) Netzeva, T., Schultz, T., Aptula, A. and Cronin, M. (2003) Partial least squares modelling of the acute toxicity of aliphatic compounds to Tetrahymena pyriformis. SAR QSAR Environ. Res. 14, 265-283.

(7) Ren, S. (2003) Ecotoxicity prediction using mechanism- and non-mechanism-based QSARs: a preliminary study. Chemosphere 53, 1053-1065. 
(8) Devillers, J. (2004) Linear versus nonlinear QSAR modeling of the toxicity of phenol derivatives to Tetrahymena pyriformis. SAR QSAR Environ. Res. 15, 237-249.

(9) Schultz, T., Netzeva, T., Roberts, D. and Cronin, M. (2005) Structure-toxicity relationships for the effects to Tetrahymena pyriformis of aliphatic, carbonyl-containing, alpha, beta-unsaturated chemicals. Chem. Res. Toxicol. 18, 330-341.

(10) Netzeva, T. and Schultz, T. (2005) QSARs for the aquatic toxicity of aromatic aldehydes from Tetrahymena data. Chemosphere 61, 1632-1643.

(11) Melagraki, G., Afantitis, A., Makridima, K., Sarimveis, H. and Igglessi-Markopoulou, 0. (2005) Prediction of toxicity using a novel RBF neural network training methodology. J Mol. Model. 8, 1-9.

(12) Roy, D., Parthasarathi, R., Maiti, B., Subramanian, V. and Chattaraj, P. (2005) Electrophilicity as a possible descriptor for toxicity prediction. Bioorg. Med. Chem. 13, 3405-3412.

(13) Aptula, A., Roberts, D., Cronin, M. and Schultz, T. (2005) Chemistry-toxicity relationships for the effects of diand trihydroxybenzenes to Tetrahymena pyriformis. Chem. Res. Toxicol. 18, 844-854.

with nonlinear methods. J. Chem. Inf. Comput. Sci. 43, 1679-1687.

(14) Ren, S. and Schultz, T. (2002) Identifying the mechanism of aquatic toxicity of selected compounds by hydrophobicity and electrophilicity descriptors. Toxicol. Lett. 129, 151-160.

(15) Deweese, AD and Schultz, T. (2001) Structure-activity relationships for aquatic toxicity to Tetrahymena: Halogen-substituted aliphatic esters. Environ. Toxicol. 16(1),54-60

(16) Cronin, M. T. D., Netzeva, T. I. ; Dearden, J. C. ; Edwards, R. ; Worgan , A. D. P., Assessment and Modeling of the Toxicity of Organic Chemicals to Chlorella vulgaris: Development of a Novel Database, Chem. Res. Toxicol. 2004, 17, 545-554. 